\title{
FLAT LIQUID CRYSTAL DIFFRACTIVE LENSES WITH VARIABLE FOCUS AND MAGNIFICATION
}

By

Pouria Valley

A Dissertation Submitted to the Faculty of the

COLLEGE OF OPTICAL SCIENCES

In Partial Fulfillment of the Requirements

For the Degree of

DOCTOR OF PHILOSOPHY

In the Graduate College

THE UNIVERSITY OF ARIZONA

2010 


\section{THE UNIVERSITY OF ARIZONA \\ GRADUATE COLLEGE}

As members of the Dissertation Committee, we certify that we have read the dissertation prepared by Pouria Valley entitled "FLAT LIQUID CRYSTAL DIFFRACTIVE LENSES WITH VARIABLE FOCUS AND MAGNIFICATION" and recommend that it be accepted as fulfilling the dissertation requirement for the Degree of Doctor of Philosophy

Date: $1 / 5 / 11$

Nasser Peyghambarian

Date: $1 / 5 / 11$

Jim Schwiegerling

Date: $1 / 5 / 11$

Gholam Peyman, MD

Final approval and acceptance of this dissertation is contingent upon the candidate's submission of the final copies of the dissertation to the Graduate College.

I hereby certify that I have read this dissertation prepared under my direction and recommend that it be accepted as fulfilling the dissertation requirement.

Date: $1 / 5 / 11$

Dissertation Director: Nasser Peyghambarian 


\section{STATEMENT BY AUTHOR}

This dissertation has been submitted in partial fulfillment of requirements for an advanced degree at The University of Arizona and is deposited in the University Library to be made available to borrowers under rules of the Library.

Brief quotations from this dissertation are allowable without special permission, provided that accurate acknowledgment of source is made. Requests for permission for extended quotation from or reproduction of this manuscript in whole or in part may be granted by the author.

SIGNED: Pouria Valley 


\title{
ACKNOWLEDGMENTS
}

I would like to thank my supervisor Professor Nasser Peyghambarian for his financial and academic support as well as Profs. Peyman and Schwiegerling for all the helpful advice and discussions.

I also like to thank the following colleagues and friends who provided lots of help and advice to me throughout the graduate school:

\author{
Majid Behabadi \\ Farrokh Shayegan \\ Reza Daj \\ Massoud Karbassian \\ Rick Franco \\ Olli Nordman \\ Nick Savidis \\ Chuck Greenlee \\ Pierre Alexander Blanche \\ Palash Gangopadhyay \\ Corry Christenson \\ Pekka Ayras \\ Josh Haddock \\ M. S. Giridhar \\ Eugene Merzylak
}




\section{DEDICATION}

I would like to dedicate this dissertation to my kind parents who have done everything for me throughout my life and I simply can't thank them enough, and to my wonderful wife who has been so loving and patient during all my long absence for finishing this project. 
TABLE OF CONTENTS

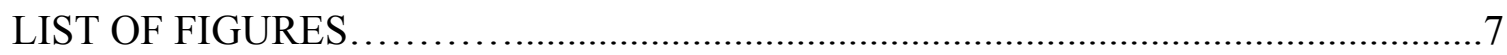

LIST OF TABLES

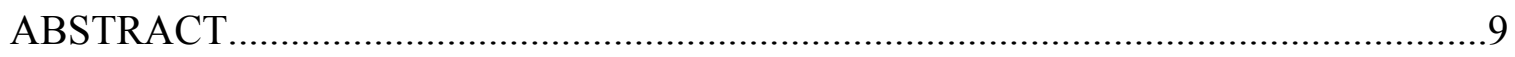

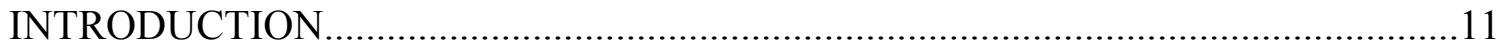

The Problem, Solution and Historical Context.......................................................11

A Review of Literature....................................................... 14

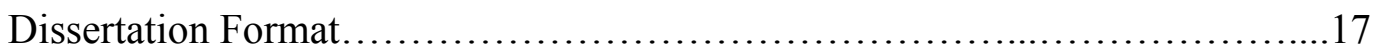

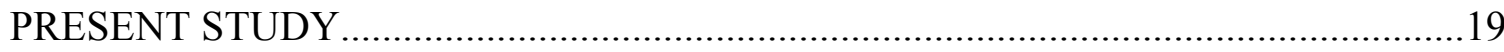

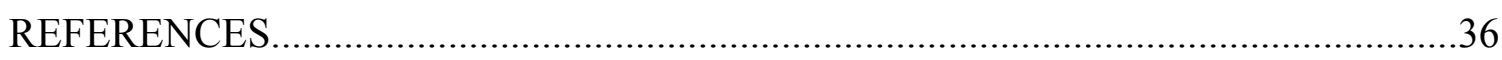

APPENDIX A: LARGE-APERTURE SWITCHABLE THIN DIFFRACTIVE LENS WITH INTERLEAVED ELECTRODE PATTERN.......................................................37

APPENDIX B: SWITCHABLE ELECTRO-OPTIC DIFFRACTIVE LENS WITH HIGH EFFICIENCY FOR OPHTHALMIC APPLICATIONS ............................40

APPENDIX C: HIGH-EFFICIENCY SWITCHABLE FLAT DIFFRACTIVE OPTHALMIC LENS WITH THREE-LAYER ELECTRODE PATTERN AND TWOLAYER VIA STRUCTURE......................................................45

APPENDIX D: TUNABLE-FOCUS FLAT LIQUID-CRYSTAL DIFFRACTIVE

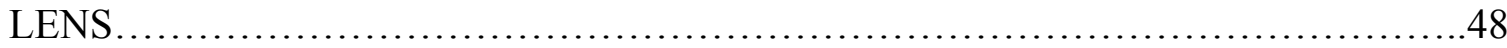

APPENDIX E: NONMECHANICAL BIFOCAL ZOOM TELESCOPE................51

APPENDIX F: ADJUSTABLE HYBRID DIFFRACTIVE/REFRACTIVE

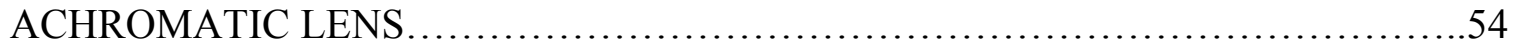

APPENDIX G: FABRICATION PROCEDURE .....................................63

APPENDIX H: A SAMPLE CODE FOR THE DESIGN AND SIMULATION OF A

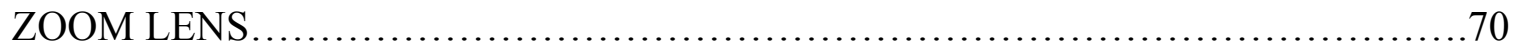

APPENDIX I: INNOVATIVE LENSES FOR CAMERA PHONES....................75 


\section{LIST OF FIGURES}

Figure $\quad$ Page

Figure 1: Comparison of optical vs. digital zoom [source: photo.net]................12

Figure 2: Electro-wetting technique........................................ 15

Figure 3: Aperture-controlled Fluidic Lens.................................. 15

Figure 4: Schematic of our flat liquid crystal diffractive lens....................... 16

Figure 5: The switchable eyeglass prototype.................................. 18

Figure 6: Binary Fresnel Zone Structure and the theoretical concept of the diffractive lens

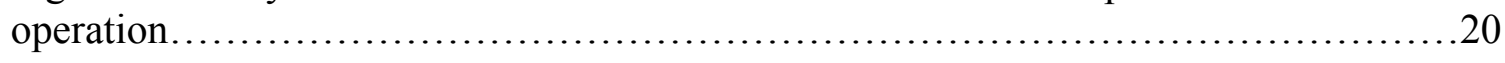

Figure 7: simulation of the liquid crystal cell at various voltage levels for an 8-level

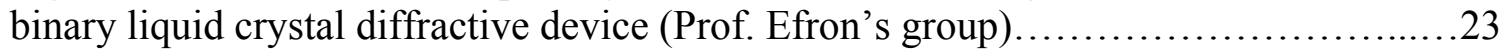

Figure 8: Measured values of the normalized amplitude versus voltage (left) and the extracted phase values versus voltage (right) ...................................... 24

Figure 9: A sample of the AutoLISP code.................................... 26

Figure 10: The Photo-mask Layout.........................................27

Figure 11: Karl-Suss mask aligner and spinner in our class-100 clean room.............29

Figure 12: The comparison between a highly contaminated (left) and a clean (right)

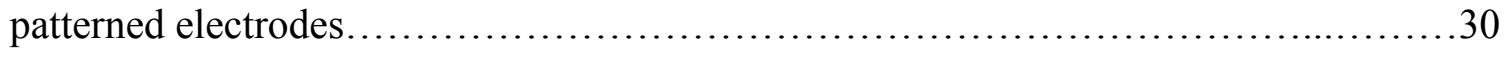

Figure 13: Edge of a fabricated lens showing the 9th bus line and the vias...............31

Figure 14: Transmission curve of one of our good 12-level lenses..................32

Figure 15: Schematic of an arbitrary zoom lens system.............................. 33 


\section{LIST OF TABLES}

Table

Page

Table 1: Competitive Advantages of our technology............................... 17

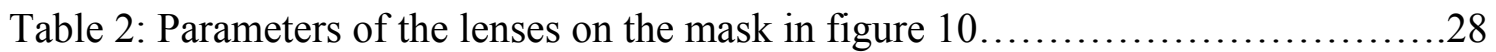




\begin{abstract}
Non-mechanical variable lenses are important for creating compact imaging devices. Various methods employing dielectrically actuated lenses, membrane lenses, and liquid crystal lenses were previously proposed [1-4]. In This dissertation the design, fabrication, and characterization of innovative flat tunable-focus liquid crystal diffractive lenses (LCDL) are presented. LCDL employ binary Fresnel zone electrodes fabricated on Indium-Tin-Oxide using conventional micro-photolithography. The light phase can be adjusted by varying the effective refractive index of a nematic liquid crystal sandwiched between the electrodes and a reference substrate. Using a proper voltage distribution across various electrodes the focal length can be changed between several discrete values. Electrodes are shunted such that the correct phase retardation step sequence is achieved. If the number of $2 \pi$ zone boundaries is increased by a factor of $m$ the focal length is changed from $f$ to $f / m$ based on the digitized Fresnel zone equation: $f=r_{m}{ }^{2} / 2 m \lambda$, where $r_{m}$ is $m^{\text {th }}$ zone radius, and $\lambda$ is the wavelength. The chromatic aberration of the diffractive lens is addressed and corrected by adding a variable fluidic lens.
\end{abstract}

These LCDL operate at very low voltage levels ( $\pm 2.5 \mathrm{~V}$ ac input), exhibit fast switching times (20-150 ms), can have large apertures $(>10 \mathrm{~mm})$, and small form factor, and are robust and insensitive to vibrations, gravity, and capillary effects that limit membrane and dielectrically actuated lenses. Several tests were performed on the LCDL including diffraction efficiency measurement, switching dynamics, and hybrid imaging with a refractive lens. Negative focal lengths are achieved by adjusting the voltages 
across electrodes. Using these lenses in combination, magnification can be changed and zoom lenses can be formed.

These characteristics make LCDL a good candidate for a variety of applications including auto-focus and zoom lenses in compact imaging devices such as camera phones. A business plan centered on this technology was developed as part of the requirements for the minor in entrepreneurship from the Eller College of Management. An industrial analysis is presented in this study that involves product development, marketing, and financial analyses (Appendix I). 


\section{INTRODUCTION}

\section{$\underline{\text { The Problem, Solution, and Historical Context }}$}

Conventional variable-focus and variable magnification (zoom lens) systems employ several fixed-focus lenses that are mechanically moved with respect to each other. This makes such optical systems bulky, expensive, fragile, and slow, with high power consumption. Reducing the weight and bulk of imaging devices is critical in applications such as mobile phones where traditional bulky systems are not suitable. As an example, there are over one billion mobile phones sold every year, and the camera is a standard feature in more than $90 \%$ of them. Apart from personal use, camera phones have become important in many areas such as journalism, business, and even law enforcement.

The first problem is lack of optical zoom which requires multiple focal lengths for the lens and is not possible with a fixed-focus lens. Figure 1 shows the importance of optical zoom. Typical camera phones in use today lack optical zoom and their picture quality is acceptable only for capturing details in a wide area (fixed focal length, object at infinity, wide field of view) but not a deep one (depth of field). As a result, typically only a few subjects appear sharp in a given image. Digital zoom is commonly available in camera phones but it degrades the resolution because it's mere electronic image processing, increasing the magnification without capturing additional pixels (i.e., no increase in optical resolution). However, with optical zoom the resolution can in fact be increased because it involves physical changes in the focal length. 


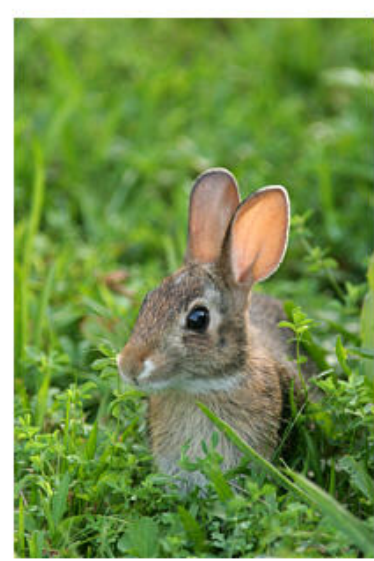

Original

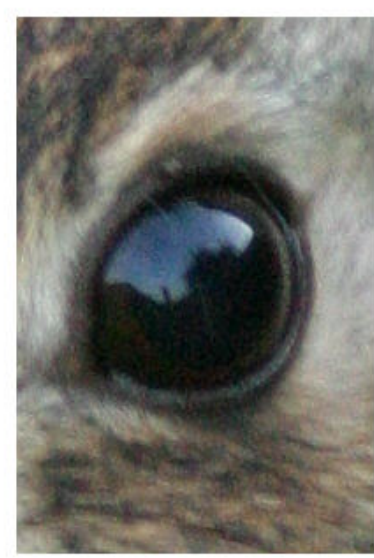

10x Optical

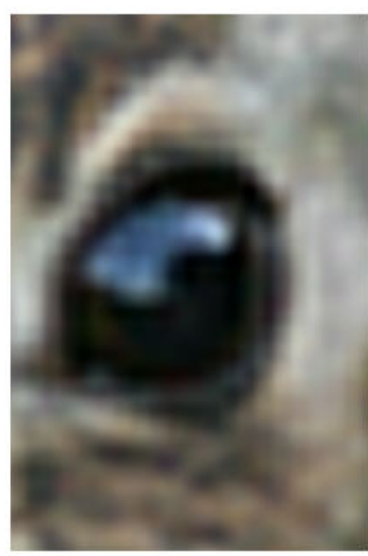

10x Digital

Figure 1: Comparison of optical vs. digital zoom [source: photo.net]

The second problem is the low image resolution, because the space constraints don't allow the focal length to be long enough (as well as detector size - number of pixels and size of pixels). Due to the short focal length of mobile phone lenses, the field of view is very wide. This means their image quality is acceptable for far subjects (wide angle shots) but is poor for near subjects (close-ups). In typical camera phones the focal length is decreased without a similar reduction in the pixel size. If the physical size constraint limits the focal length of a lens, then minimum pixel size limits the resolution of the image. Providing longer focal lengths results in greater magnification and improved angular resolution. Liquid crystal diffractive lenses presented here are flat and compact (can be made less than $1 \mathrm{~mm}$ thick), low cost (mass-producible), rugged, fast (no mechanical movements), and consume little power.

The history behind the creation of the variable liquid crystal diffractive lens dates back to 1980 [5]. Sam Sato published a paper in that year that demonstrated, for the first 
time, the use of a liquid crystal in conjunction with a diffractive optical element called a Fresnel lens. This was a radical innovation in the field of optical sciences that subsequently led to several incremental innovations. Before that time, any variable optical system had to incorporate several fixed-focus lenses that are moved with respect to each other to vary the overall focal length of the system. Sato et al demonstrated a surface relief (like saw tooth) Fresnel lens which had a fixed focal length. However, the effective refractive index of the liquid crystal was varied to achieve a multi-focal lens. This lens had a relatively low diffraction efficiency as well as low transmission and long response time due to the large thickness of the liquid crystal. An incremental innovation led to a solution for the transmission and response time issues by reducing the thickness of the liquid crystal. The new technology used a flat Fresnel zone plate instead of the surface relief Fresnel lens. However, the diffraction efficiency was still low because in the Fresnel zone plates, every other zone (either the odd or even zones) are blocked to achieve a constructive interference at the image plane. Another incremental technology took place afterward which used the liquid crystal in conjunction with a refractive lens (instead of the diffractive lens). This allowed for better efficiency but still the problem of long response time and low transmission due to the absorption and scattering of a thick liquid crystal layer was there. Plus, in all of the abovementioned innovations the lens exhibited a non-zero optical power which makes them not suitable for many applications including ophthalmic applications. In addition, our lenses have zero optical power at the off (no-voltage) state which is desirable in many applications such as vision care. This was an important incremental innovation that was built on the original innovation by Sam Sato which overcame many of the problems associated with the earlier concepts. 
Our innovation took place at the College of Optical Sciences at the University of Arizona in 2003. In a group effort, we designed, fabricated, and characterized flat liquid crystal diffractive lenses with high diffraction efficiency and transmission, and fast response time. In addition, our lenses have zero optical power at the off (no-voltage) state which is desirable in many applications such as vision care. This was an important incremental innovation that was built on the original innovation by Sam Sato which overcame many of the problems associated with the earlier concepts.

\section{$\underline{\text { A Review of the Literature }}$}

A few other methods of making compact non-mechanical variable lenses are currently under investigation including non-diffractive liquid-crystal lenses, electrowetting lenses, and membrane fluidic lenses. In the electro-wetting technique [6-10], two immiscible liquids such as oil and water are used (Figure 2). By applying a voltage across the liquids, the curvature of interface and hence the focal length is changed. The required voltages are in the order of several tens or even over a hundred volts which makes this method not very suitable for battery-run applications such as camera phones. Also, the practical aperture size for electro-wetting lenses is small due to the gravitational forces. 

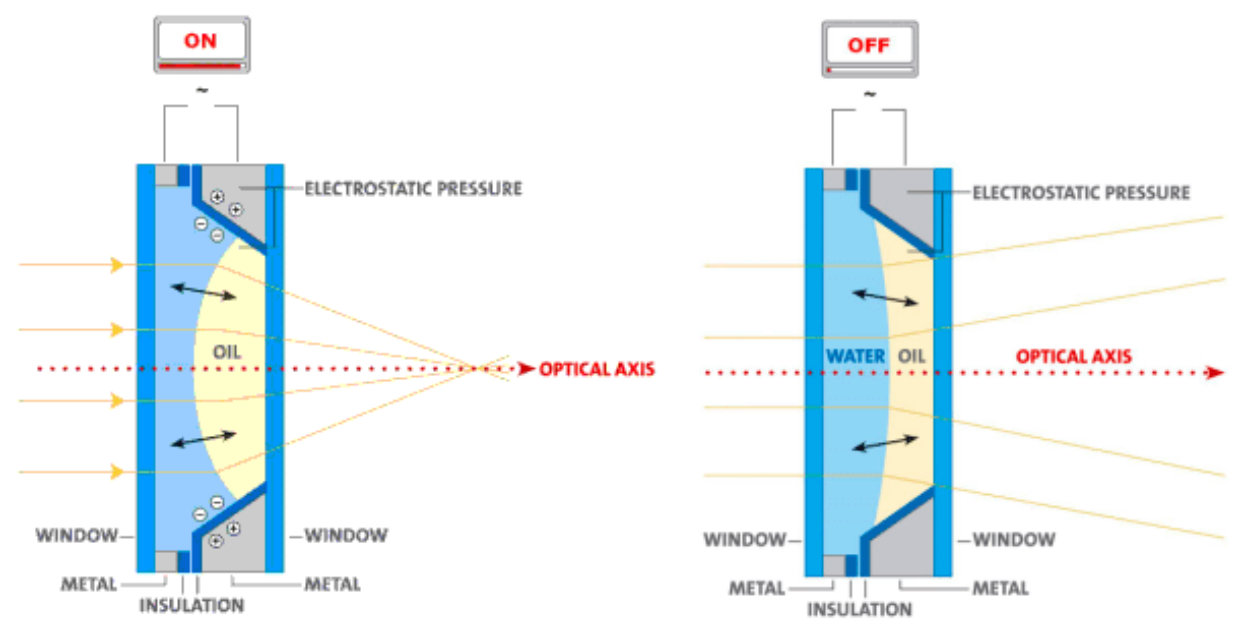

Figure 2: Electro-wetting technique.

The fluidic lenses [11] normally employ a deformable PDMS membrane chamber. Pressure-controlled fluidic lenses use a syringe and a pump system to alter the volume of the fluid inside the chamber and hence vary the focal length (Figure 3). Another type of fluidic lens utilizes a servo motor to change the aperture of the lens while the volume of the fluid is constant. Alignment, evaporation, slow response time, and bulky peripherals are some of the current issues with the fluidic lenses.

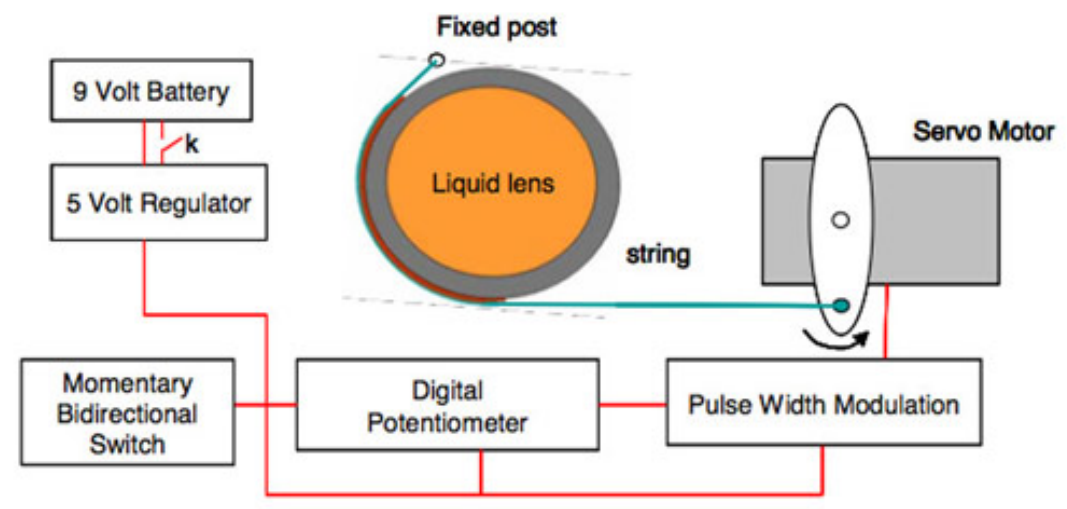

Figure 3: Aperture-controlled Fluidic Lens. 
Our lens modules as depicted in Figure 4 are comprised of two main components: a flat diffractive element, and a thin layer of liquid crystal sandwiched between two thin ITO glass substrates, one with the diffractive pattern and one with no pattern used as the electronic ground. The refractive index of the liquid crystal can be varied with the applied voltage and together with the diffractive pattern that defines the phase-wrap points, phase profiles corresponding to different focal lengths can be achieved.

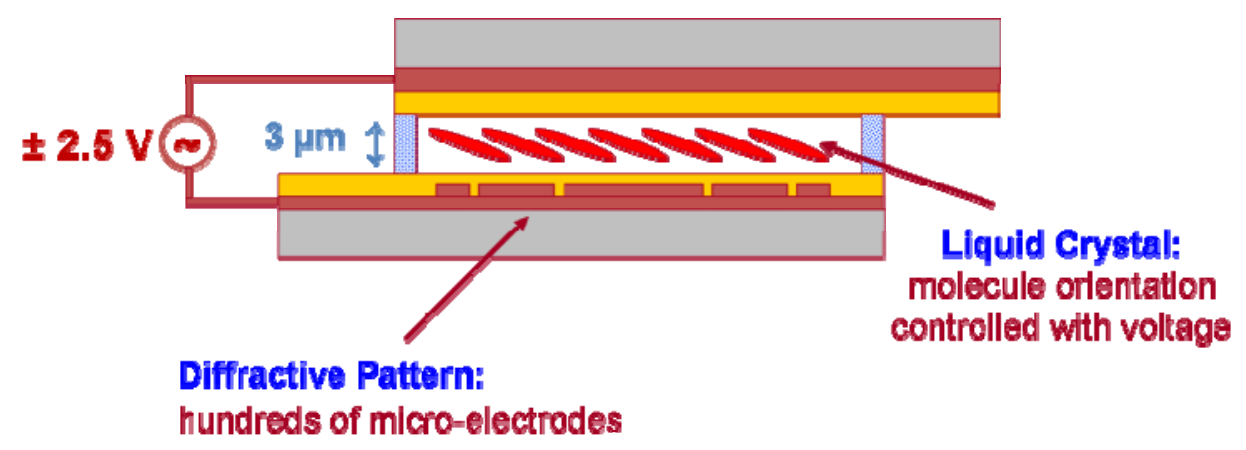

Figure 4: Schematic of our flat liquid crystal diffractive lens

The main advantages of our liquid-crystal diffractive lenses compared to the other technologies include low power consumption (operating voltages of less than $2 \mathrm{~V}_{\text {rms }}$ compared to several tens of volts for the other lenses), scalability of the lens aperture (up to $15 \mathrm{~mm}$ or more, whereas the other lenses are limited to about $5 \mathrm{~mm}$ or less), fast response time (about $50 \mathrm{~ms}$ compared to seconds for thick liquid-crystal or fluidic lenses), compactness (no need for a pump machine, actuator, or servo motor compared to the elastic membrane lenses), long-term stability and robustness (insensitivity to vibrations, gravity, and capillary effect as in membrane and electro-wetting lenses), failsafe mode (no optical power when the power in turned off, unlike most other lenses), and flatness. Table 1 summarizes a few important advantages of our technology (LenSense is 
a working title for our business venture) as compared to electro-wetting (Varioptic) and fluidic lenses (Holochip).

\begin{tabular}{|c|c|c|c|}
\hline Feature Company & Len'Sense & VariOptic & Holochip \\
\hline $\begin{array}{l}\text { Power Consumption } \\
\text { Maximum Operating Voltage }\end{array}$ & $\begin{array}{c}\text { Low } \\
2 \mathrm{~V}\end{array}$ & $\begin{array}{l}\text { High } \\
60 \mathrm{~V}\end{array}$ & $\begin{array}{l}\text { High } \\
250 \mathrm{~V}\end{array}$ \\
\hline $\begin{array}{l}\text { Aperture Size } \\
\text { Practical Diameter }\end{array}$ & $\underset{15 \mathrm{~mm}}{\operatorname{Large} \checkmark}$ & $\begin{array}{l}\text { Small } \\
5 \mathrm{~mm}\end{array}$ & $\begin{array}{l}S \mathrm{Small} \\
5 \mathrm{~mm}\end{array}$ \\
\hline $\begin{array}{c}\text { Sensitivity } \\
\text { Gravity, Shock, Vibrations }\end{array}$ & $\begin{array}{c}\text { Low } \checkmark \\
\text { Liquid-Crystal }\end{array}$ & $\begin{array}{c}\text { High } \\
\text { oil and Water }\end{array}$ & $\begin{array}{c}\text { High } \\
\text { Pollmeric Liquid }\end{array}$ \\
\hline $\begin{array}{l}\text { Form Factor } \\
\text { Needed Edge Space }\end{array}$ & $\underset{1 \mathrm{~mm}}{\text { Small } \checkmark}$ & $\begin{array}{l}\text { Medium } \\
5 \mathrm{~mm}\end{array}$ & $\begin{array}{l}\text { Large } \\
39 \mathrm{~mm}\end{array}$ \\
\hline
\end{tabular}

Table 1: Competitive Advantages of our technology

\section{$\underline{\text { Dissertation Format }}$}

The format of dissertation is based on six journal papers attached in the appendices. Over the past seven years, we have demonstrated four different generations of liquid crystal diffractive lenses in a group effort. The dissertation author had an assistive role during the first two generations which employed 4- and 8- binary phase level Fresnel zones. For the last two generations that utilized 12- and 16- binary phase level Fresnel zones and added several important improvements, the author performed the whole design and simulations, as well as most of the fabrication and testing as outlined in the next chapter in detail.

The first two generations were designed mainly for the vision care applications including correction of Presbyopia and making a switchable eyeglass. We successfully 
demonstrated a proof-of-principle prototype for vision applications including a switchable bifocal eyewear, which was a revolution in the eyeglass industry after 243 years of relatively little change (Figure 5). The last two generations were geared towards auto-focus and zoom lens applications that were proposed by the author who also filed a provisional patent application for that. He also developed a business plan with the collaboration of his entrepreneurship mentor and two classmates and took part in "Mobile Rules" innovation competition and was selected as a finalist among one hundred companies worldwide.

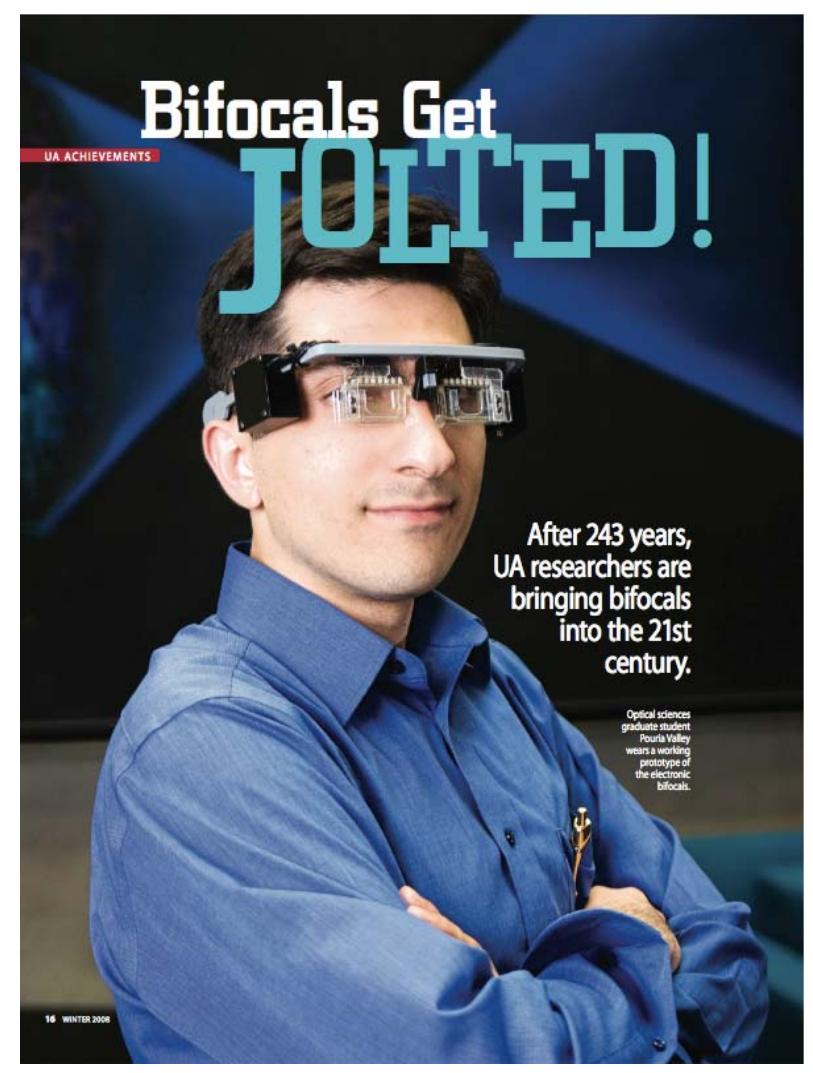

Figure 5: The switchable eyeglass prototype. 


\section{PRESENT STUDY}

The methods, results, and conclusions of this study are presented in the journal papers appended to this dissertation. The following is a summary of the most important findings as well as the explanation of the flow of the attached papers in this document.

The theoretical concept and operating mechanism of our liquid crystal diffractive lenses are first presented, separately. Then the fabrication and characterization methods are discussed in this section as well as the six papers in the appendices. Our lenses are comprised of a flat diffractive optical element (DOE) and a thin layer of liquid crystal sandwiched between two thin ITO glass substrates, one with the DOE pattern (Figure 4) and one with no pattern used as the electronic ground. An electric filed is applied across a thin film of mono-domain, aligned, nematic liquid-crystal (E7 from Merk) by accurate voltages applied to the patterned electrodes to vary the effective refractive index and hence produce a diffractive optical element (DOE). Each Fresnel zone is divided to multiple sub-zones (eight in Figure 6) in a way that maintains the square-root-of-radius relationship so as to quantize the continuous quadratic phase transmission function:

$$
r_{m, n}=\sqrt{2[(m-1)+n / L] \lambda f}, \quad n=1,2, \ldots, L
$$

where $\mathrm{n}$ is an index denoting the $\mathrm{n}^{\text {th }}$ boundary, and $\mathrm{m}$ is an index denoting the boundries of the sub zones, and $\mathrm{f}$ is the distance of the plane from center of the spherical wave that is the focal length of the lens, and $\lambda$ is the wavelength of the light. 

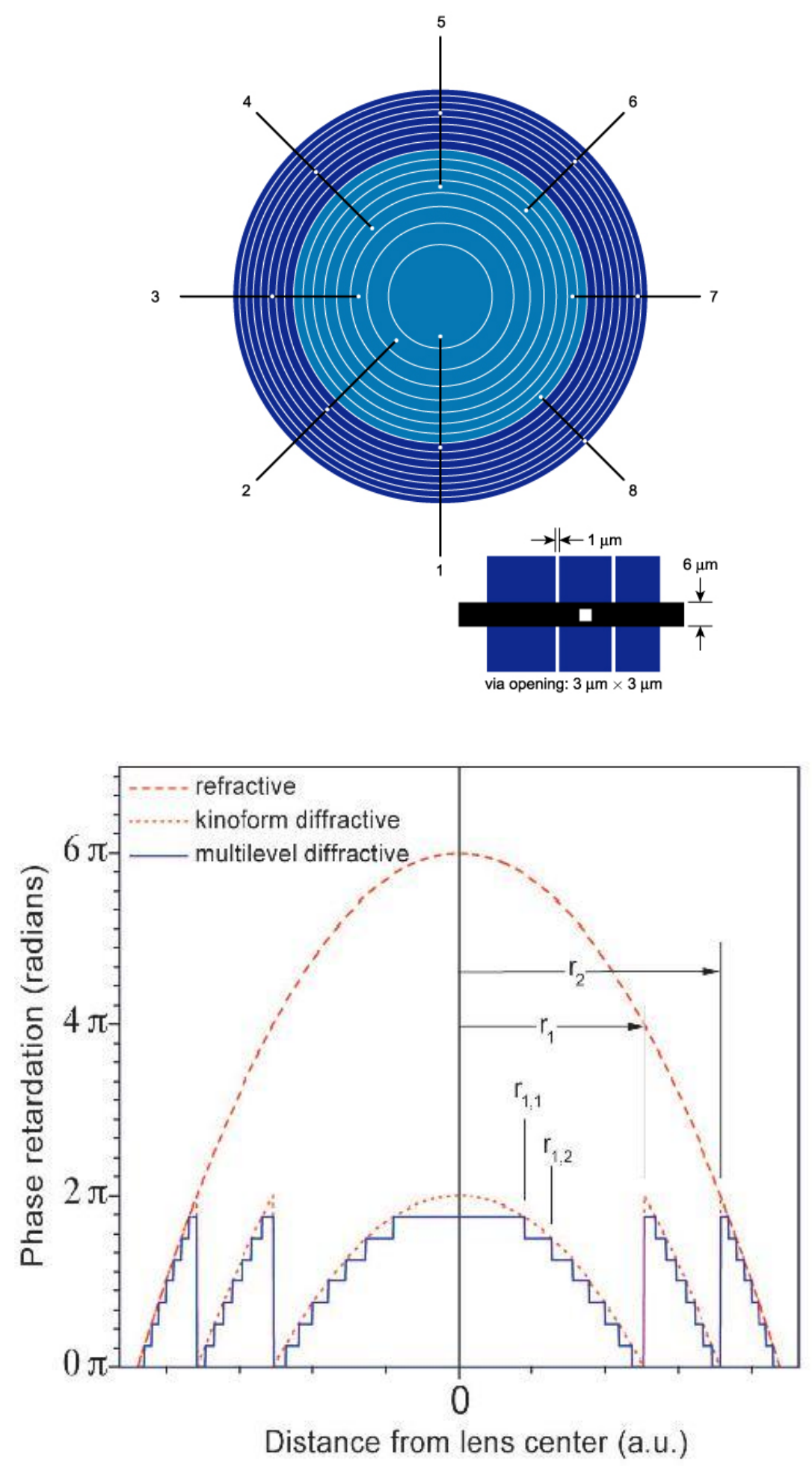

Figure 6: Binary Fresnel Zone Structure and the theoretical concept of the diffractive lens operation 
The electrical buses are established to shunt together all of the corresponding sub-zones in all of the zones. The electrodes of the same counting index for each subzone within each of the Fresnel zones are connected to bus bars through vias made in the insulating layer of $\mathrm{SiO}_{2}$. Isolation between the bus bars and the electrodes is achieved by a thin insulating layer of $\mathrm{SiO}_{2}$ that contains two micron vias only at proper electrode locations where electrical contacts between the electrodes and corresponding bus bars are needed (Figure 6). The focusing power of the lens can be switched between some specially related discrete values. The phase wrapping points can be re-established if the number of subzones is changed. The electrodes have fixed positions but the phase retardation they produce can be reset by changing the number of binary subzones. The effective refractive index of the liquid crystal can be varied with the applied voltage and together with the diffractive pattern that defines the phase-wrap points, phase profiles corresponding to various focal lengths can be achieved.

Liquid crystal is a phase of matter in between an isotropic liquid and a crystalline solid. In liquid crystal molecules, there is no positional order (as in liquids) but there exists a certain degree of orientational order (as in crystalline solids). Liquid crystals can be categorized in three different materialistic phases in general: smetic, choletric, and nematic. The nematic liquid crystal which is considered in this dissertation is characterized by a single axis of symmetry. In nematic liquid crystals, molecules form a uniaxially symmetric medium in which the axis of symmetry is determined by a director unit vector that represents the average orientation of the molecules. When the long axes of the liquid crystal molecules are parallel to the plane of the substrate (due to the specific 
rubbing direction) the alignment is called homogeneous which is our alignment of choice. In this type of alignment the device is said to operate in the electrically controlled birefringence (ECB) mode which is the most often used method.

The two principal refractive indices of a nematic liquid crystal are called the ordinary $\left(\mathrm{n}_{\mathrm{o}}\right)$ and extra-ordinary $\left(\mathrm{n}_{\mathrm{e}}\right)$ refractive indices. In the ECB operation mode a linearly polarized incoming light propagating parallel to the optic axis (LC director) will undergo the low voltage extra-ordinary $\left(\mathrm{n}_{\mathrm{e}}\right)$ refractive index and experiences ElectroOptic phase modulation while a light propagating perpendicular to the optic axis will experience the high voltage ordinary $\left(\mathrm{n}_{\mathrm{o}}\right)$ refractive index and will undergo no electrooptic effects. The birefringence of the liquid crystal is defined as the difference between the ordinary and extra-ordinary refractive indices. For E7 at room temperature and at the $555 \mathrm{~nm}$ wavelength:

$$
\Delta \mathrm{n}=\mathrm{n}_{\mathrm{e}}-\mathrm{n}_{\mathrm{o}}=1.75-1.52=0.23
$$

A simulation of how the liquid crystal molecules are oriented at various voltage levels is shown in Figure 7 for an 8-level binary liquid crystal diffractive device. The values of the refractive indices and phase retardations are calculated for a 5 micron thick layer of E7 nematic liquid crystal. As seen in this figure, at the threshold voltage of 1.1 volt the molecules are still almost aligned with the original orientation dictated by the buffing direction of the liquid crystal's alignment layer on the substrate. At these low voltage levels the light experiences the extra-ordinary refractive index of 1.7 and as the voltage is increased the molecules start to rotate and become more perpendicular to the initial director orientation and the light experiences the ordinary refractive index of 1.5. 


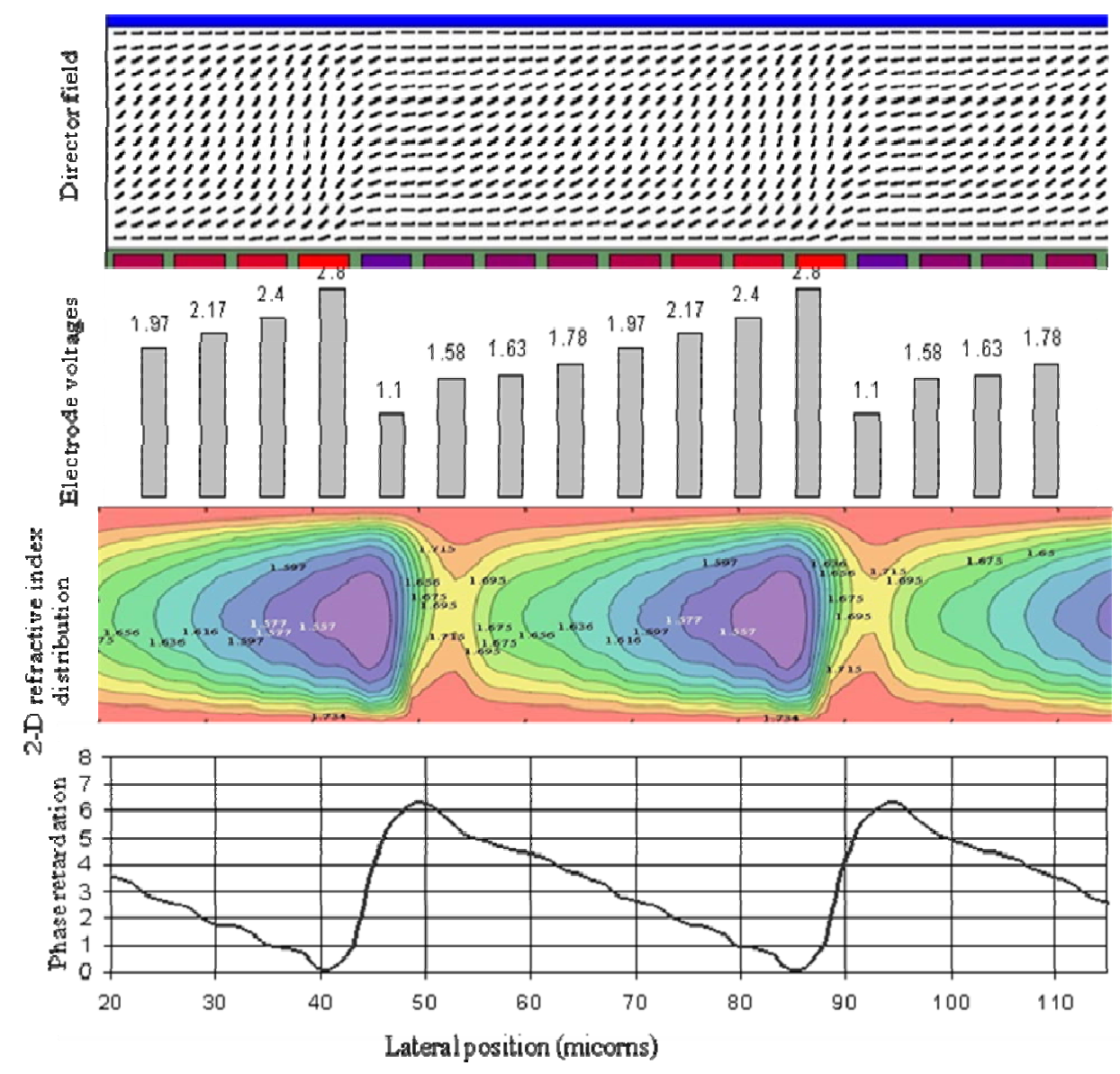

Figure 7: simulation of the liquid crystal cell at various voltage levels for an 8-level binary liquid crystal diffractive device (Prof. Efron's group)

The amplitude response of a liquid crystal cell versus voltage was measured using a photo detector and plotted as seen in Figure 8 (left). In this chart, the absolute amplitudes were normalized by the initial incident intensity. The phase measurement would be more complex than measuring the amplitude, and can be done using interferometric techniques. However, the phase values can easily be extracted from the registered amplitude response through the following equations: 


$$
\begin{gathered}
I=I_{0} \cos ^{2} \frac{\Gamma}{2} \\
\Gamma=2 \cos ^{-1} \sqrt{\frac{I}{I_{0}}}
\end{gathered}
$$
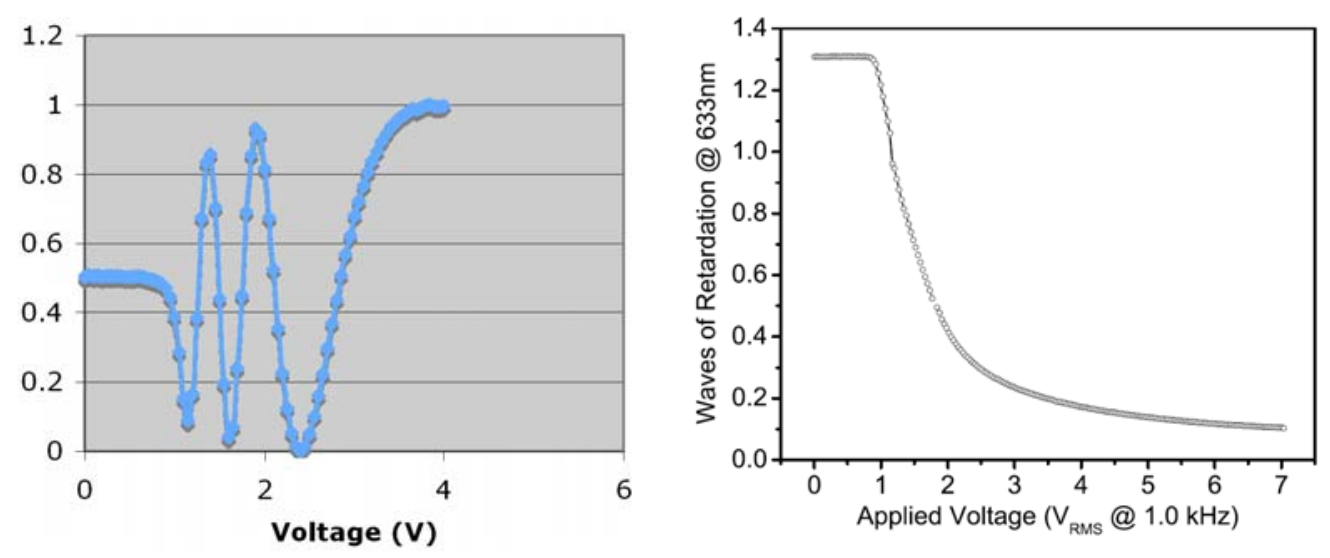

Figure 8: Measured values of the normalized amplitude versus voltage (left) and the extracted phase values versus voltage (right)

The general analytical expression for the phase response below can also be used to calculate an approximate theoretical phase response:

$$
\Gamma(V)=\frac{\pi d}{2 \lambda}\left(n_{e}-n_{o}\right)\left(\frac{n_{e}^{2}}{n_{o}^{2}}+\frac{n_{e}}{n_{o}}\right)\left(\frac{V^{2}-V_{t h}^{2}}{\frac{2}{3} V^{2}+\frac{K_{3}-K_{1}}{K_{1}} V_{t h}^{2}}\right)
$$


Our first three papers were centered on the ophthalmic applications. The first paper titled "Large-aperture switchable thin diffractive lens with interleaved electrode patterns" (Appendix A) describes the concept, design, fabrication, and test results from the first generation of our lenses that had 4- binary phase levels for the Fresnel zone structure. Our second journal paper "Switchable electro-optic diffractive lens with high efficiency for ophthalmic applications" (Appendix B) reports on an 8-level lens with higher diffraction efficiency with more detailed tests. We also demonstrated a switchable eyewear prototype in this paper for the vision care industry. The third paper "Highefficiency switchable flat diffractive ophthalmic lens with three-layer electrode pattern and two-layer via structures" (Appendix C) describes a modification in the fabrication method that makes it easier to fabricate lenses with higher number of phase levels, but the number of fabrication steps is increased. Our last three papers are geared towards the auto-focus and zoom lens applications, as well as correcting the chromatic aberration of our liquid crystal diffractive lens by adding a pressure-controlled fluidic lens. These papers are discussed in more detail in this section and covers materials which could not be included in the papers due to the space constraints.

Our fourth paper titled "Tunable-focus flat liquid crystal diffractive lens" (Appendix D) demonstrates a 12-level lens with a diffraction efficiency of up to $95 \%$ that can be tuned between five discrete focal lengths. The first step is to design the diffractive element with computer aided design (CAD) software, such as AutoCAD. AutoLisp code was used to generate the electrodes. For the first two generations, the vias and bus lines were drawn manually. This was not a problem when dealing with small number of phase levels, and small number of lenses per mask. However, for the next two generations of 
lenses the vias and electrodes were also included in the code so the program automatically draws them. Figure 9 shows a part of this multi-hundred line code.

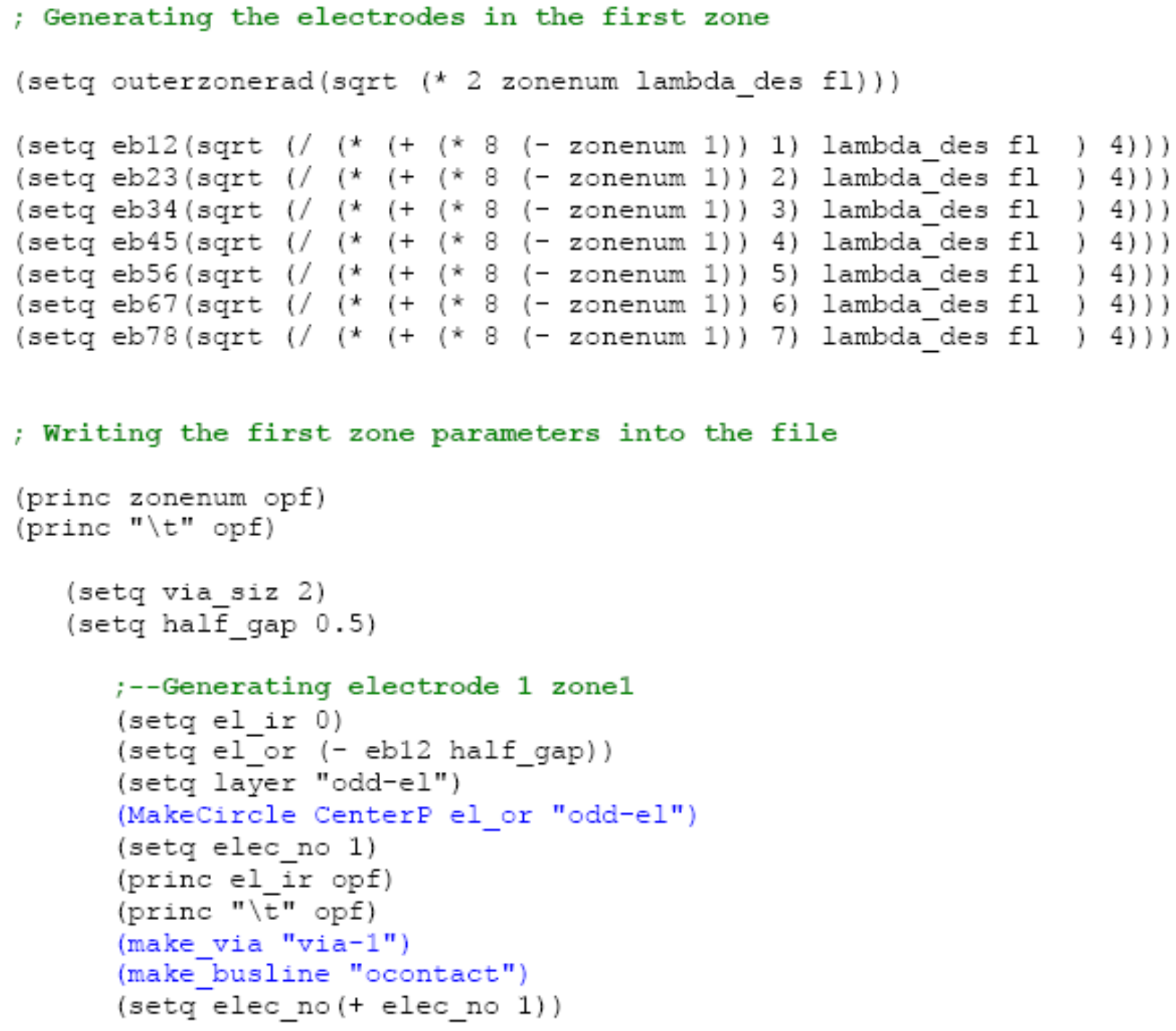

Figure 9: A sample of the AutoLISP code.

Additional features such as alignment marks and etch test structures are drawn manually. The DXF file generated by AutoCAD is converted into the GDSII format which is sent to the mask manufacturer. The latest generation of our masks is depicted in Figure 10 that 
contains 17 lenses with various optical powers and apertures and phase levels as summarized in Table 2.

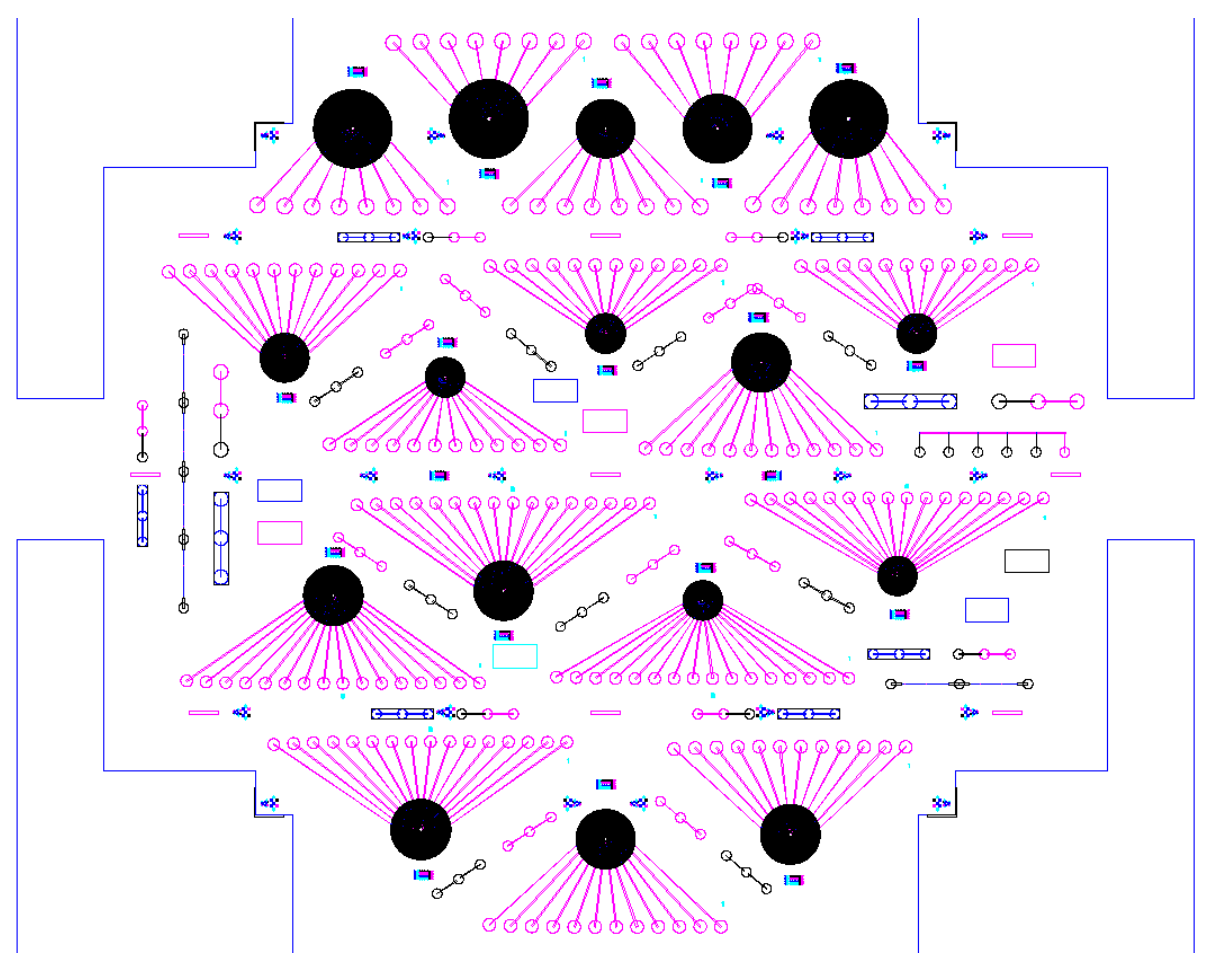

Figure 10: The Photo-mask Layout. 


\begin{tabular}{|c|c|c|c|c|c|}
\hline Phase Levels & $\begin{array}{c}\text { Aperture Dia. } \\
(\mathrm{mm})\end{array}$ & $\begin{array}{c}\text { Power } \\
(\mathrm{D})\end{array}$ & $\begin{array}{c}\text { Narrowest Ring } \\
\text { (micron) }\end{array}$ & $\begin{array}{c}\text { Gap Sizes } \\
\text { (micron) }\end{array}$ & $\begin{array}{c}\text { Via Sizes } \\
\text { (micron) }\end{array}$ \\
\hline \multirow{3}{*}{16} & 4 & 3.4 & 0.8 & 1.5 \\
\cline { 2 - 6 } & \multirow{3}{*}{16} & 3 & 4.7 & 1 & 2 \\
\cline { 2 - 6 } & \multirow{3}{*}{6} & 2.5 & 3.7 & $1,0.8$ & 1.8 \\
\cline { 3 - 6 } & \multirow{2}{*}{6} & 2 & 4.6 & 1 & 2 \\
\cline { 3 - 6 } & & 1.5 & 6.1 & $1.8,1.4$ & 2 \\
\hline
\end{tabular}

\begin{tabular}{|c|c|c|c|c|c|}
\hline \multirow{7}{*}{12} & \multirow{3}{*}{4} & 5 & 3.7 & 0.8 & 1.8 \\
\hline & & 4.5 & 4 & 1 & 1.8 \\
\hline & & 4 & 4.7 & 1 & 2 \\
\hline & 5 & 3.5 & 4.2 & $1.2,1$ & 1.8 \\
\hline & \multirow{3}{*}{6} & 3 & 4.3 & $1.2,0.8$ & 2 \\
\hline & & 2.5 & 5.1 & 1 & 2 \\
\hline & & 2 & 6.1 & 1.4 & 2 \\
\hline
\end{tabular}

\begin{tabular}{|c|c|c|c|c|c|}
\hline \multirow{3}{*}{8} & 6 & 5 & 3.8 & $1,0.8$ & 1.5 \\
\cline { 2 - 6 } & 7 & 4 & 3.9 & 1 & 1.8 \\
\cline { 2 - 6 } & \multirow{3}{*}{8} & 3 & 4.7 & 1 & 2 \\
\cline { 2 - 6 } & \multirow{2}{*}{8} & 2.5 & 5.6 & 1.2 & 2 \\
\cline { 2 - 6 } & & 2 & 7.1 & 1.6 & 2 \\
\hline
\end{tabular}

Table 2: Parameters of the lenses on the mask in figure 10.

In the next step, diffractive patterns are lithographically transferred and etched into the thin layers of conductive and transparent indium tin oxide (ITO) coated on a glass substrate. The fabrication techniques for these diffractive patterns are very similar to those used in the semiconductor industry. These processes require high quality lithography equipment and tight environmental control in terms of contamination, 
temperature, humidity, etc. (clean room of Class 100 or better.) Figure 11 shows one of the Class 100 clean rooms at the University of Arizona.

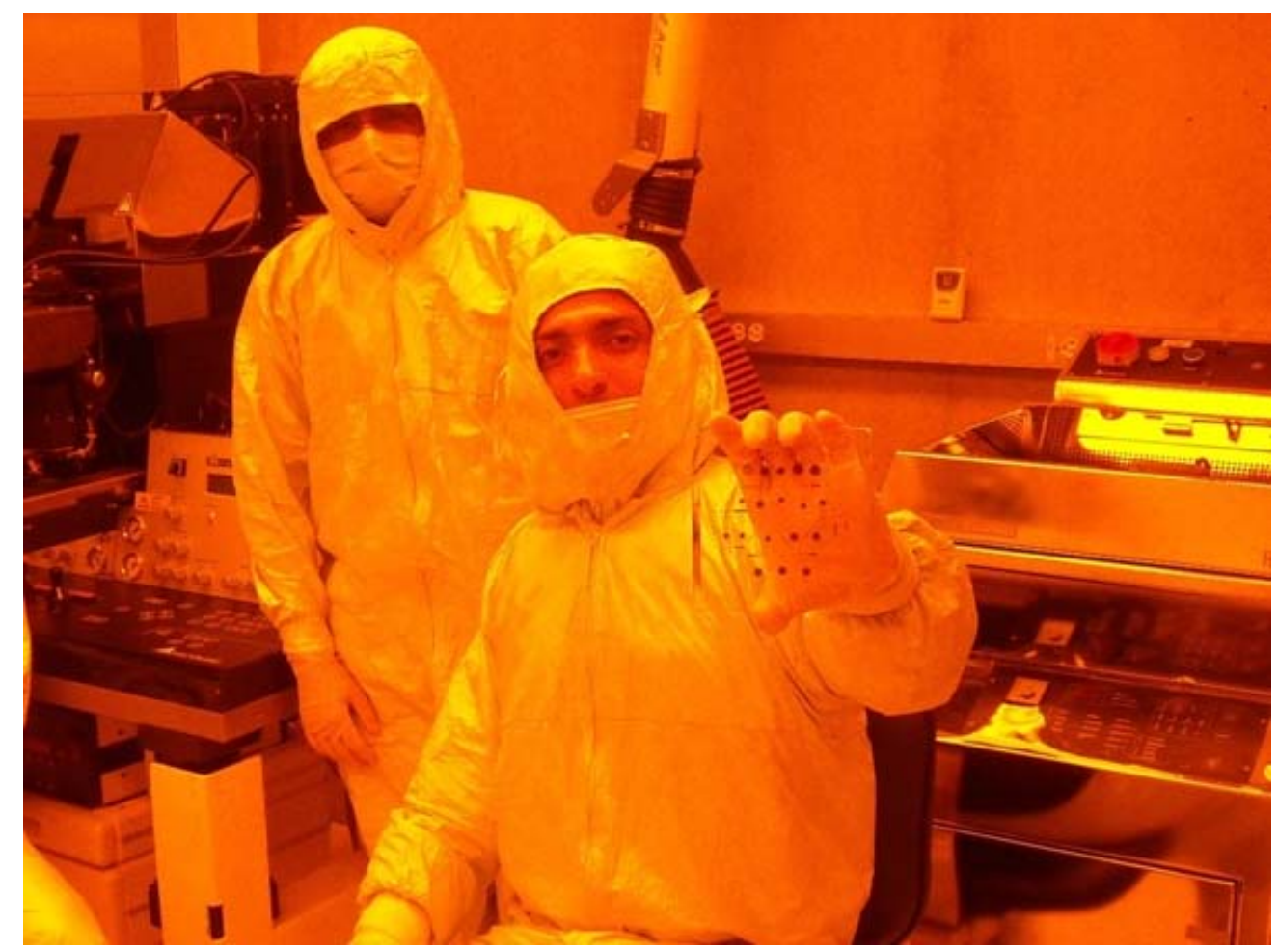

Figure 11: Karl-Suss mask aligner and spinner in our class-100 clean room.

The micro-fabrication of our lenses was by far the most cumbersome and time consuming part of the projects. This was in part due to the very small feature sizes (down to 0.8 micron) that are difficult to make with conventional contact printing lithography. But the more important obstacles were the deviations of our clean room environment and its tools from the industry standards. As an example, contamination levels were often too high mainly due to the neglect of other users. Figure 12 shows a comparison between two patterned electrodes with heavy (left) and no (right) contamination. Even at lighter 
contamination levels which we frequently observed, the chance of problem and having to redo the lithography for that layer would be high because even a very small particle of size 1 micron or higher could cause a short between the two adjacent ITO electrodes. We also encountered problems with some of the tools and machines. Most recently the quality of the Silicon Dioxide deposited by our sputtering system was low, and so we explored alternatives such as CVD and spin-on glass.
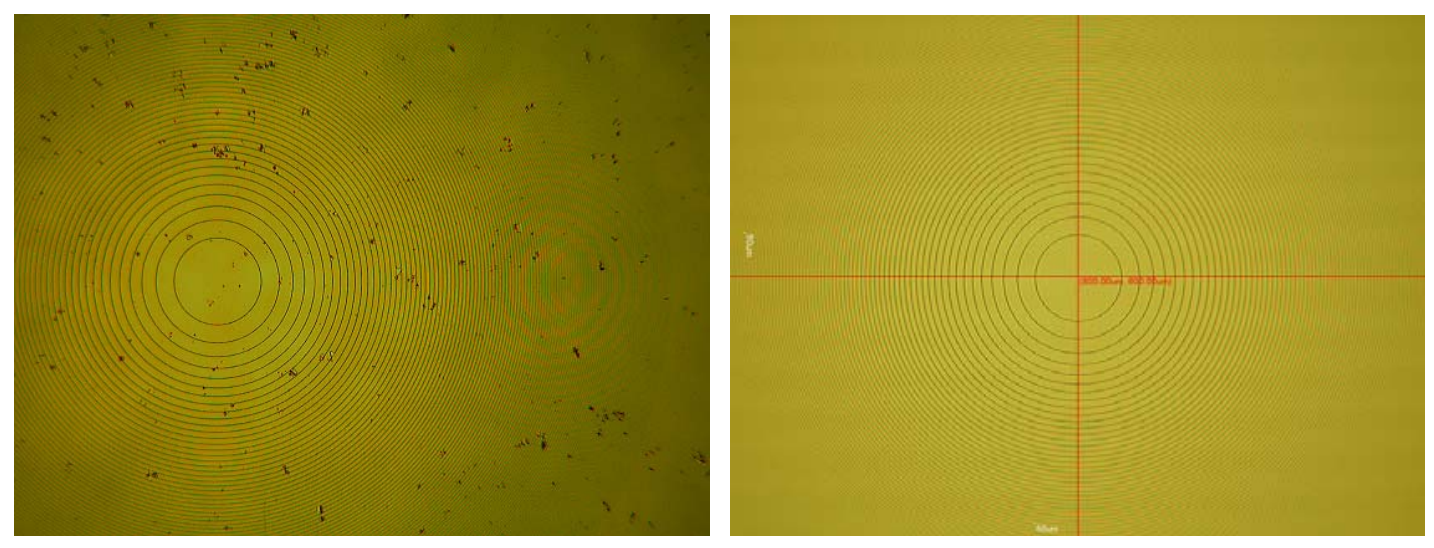

Figure 12: The comparison between a highly contaminated (left) and a clean (right) patterned electrodes.

The complete fabrication procedure is attached in Appendix G. Figure 13 shows a portion of one of the good lenses we fabricated with all the layers being clean and working. 


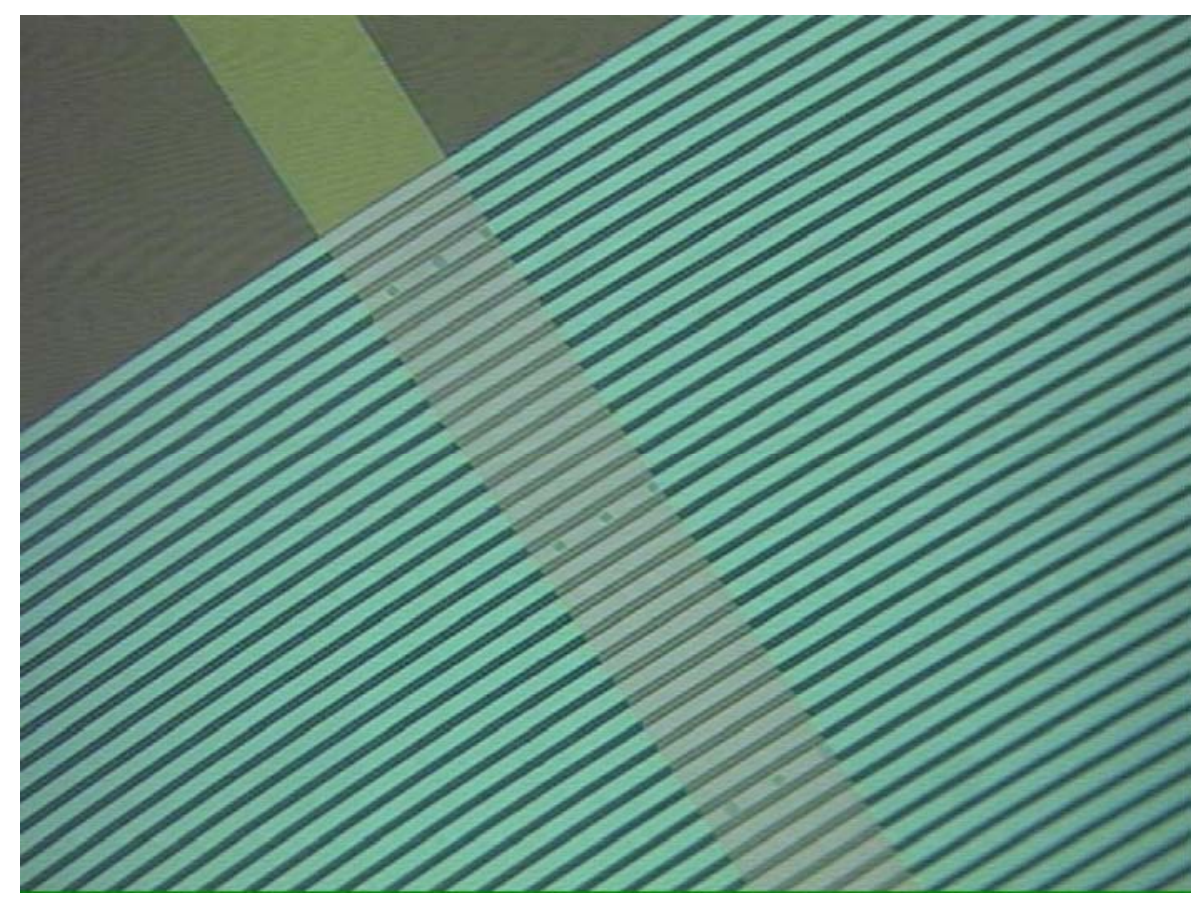

Figure 13: Edge of a fabricated lens showing the 9th bus line and the corresponding vias

After successful fabrication of the diffractive lens, a cell is formed and filled with liquid crystal as described in the papers as well as Appendix F in more detail. We tried a few different thicknesses for the liquid crystal layer (from 15 micron to 3 micron). The minimum required thickness for the liquid crystal in order to have a full $2 \pi$ phase change can be found from the following equations:

$$
\Delta \phi_{\max }=\frac{2 \pi}{\lambda} d \Delta n_{\max }=2 \pi \quad d_{\min }=\lambda / \Delta n_{\max }
$$

For the E7 (Merk) liquid crystal that we used $\Delta \mathrm{n}=0.225$, so at $\lambda=555 \mathrm{~nm}$ the minimum required thickness would be $2.5 \mu \mathrm{m}$.

Transmission curves of our assembled lenses were tested by a spectrophotometer to measure the thickness of the cell gap and also characterize the transmission of the lens 
at different wavelengths (Figure 14). The transmission is about $80 \%$ on average over the visible range but it drops as we move toward infrared or ultraviolet ranges due to the high absorption of the ITO glass. The transmission can be further increased by optimizing the thicknesses of the ITO and $\mathrm{SiO}_{2}$ films as well as employing anti-reflection coatings.

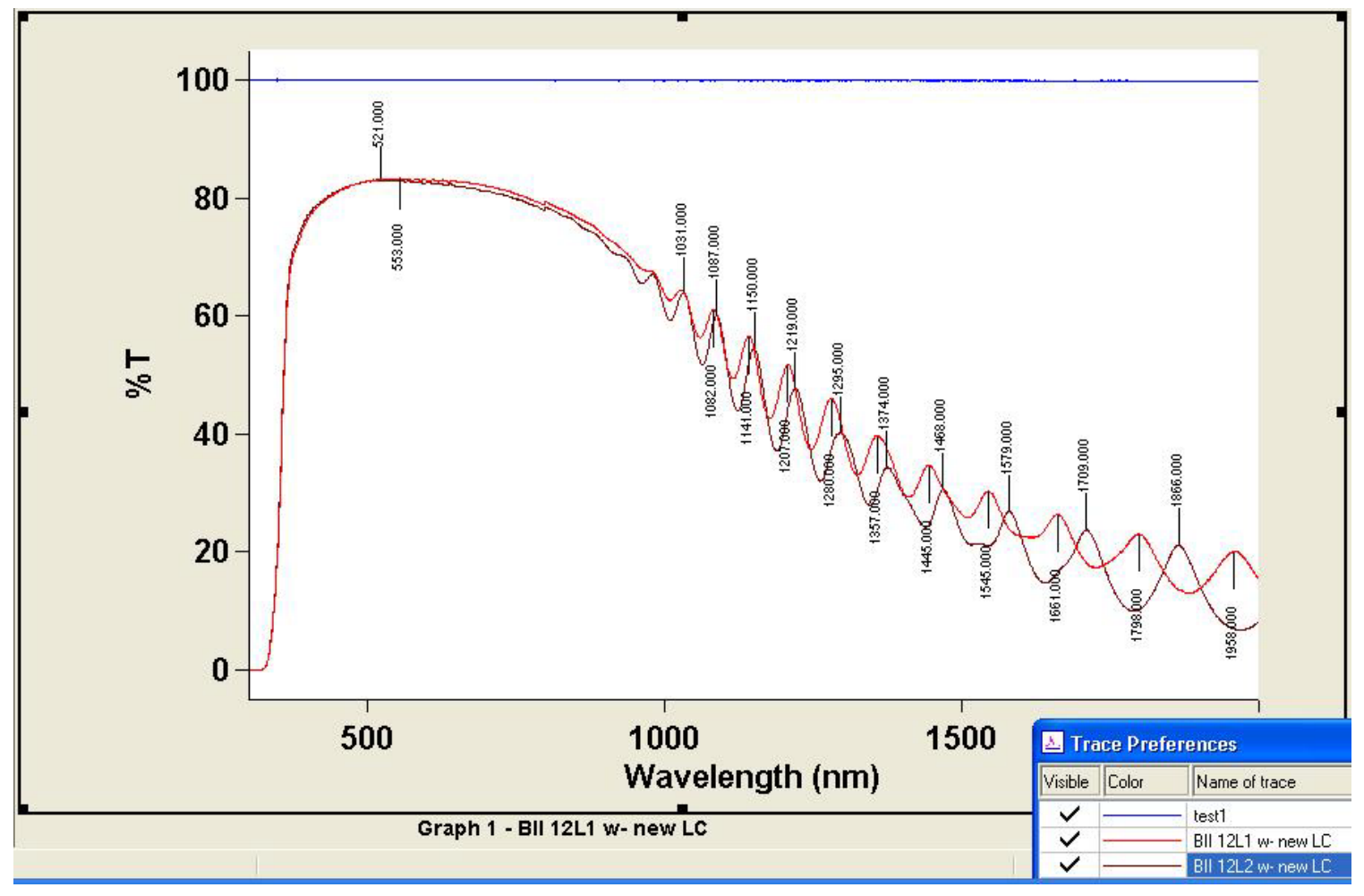

Figure 14: Transmission curve of one of our good 12-level lenses.

Several tests were performed to characterize the performance of our lenses including diffraction efficiency measurements, ellipsometry (polarization microscopy), interferometry, response time measurements, and various hybrid imaging. The methods are discussed in detail in the attached papers and the results are presented which show good performance for our lenses. 
In our fifth paper "Non-mechanical Bifocal Zoom Telescope" we demonstrated a variable magnification system using two of our liquid crystal lenses in combination. We designed the system based on a telescopic configuration but we could also make a zoom lens in an arbitrary configuration as shown if figure 15. This system employs two variable liquid crystal diffractive lenses and a fixed-focus static lens to increase to overall optical power of the system.

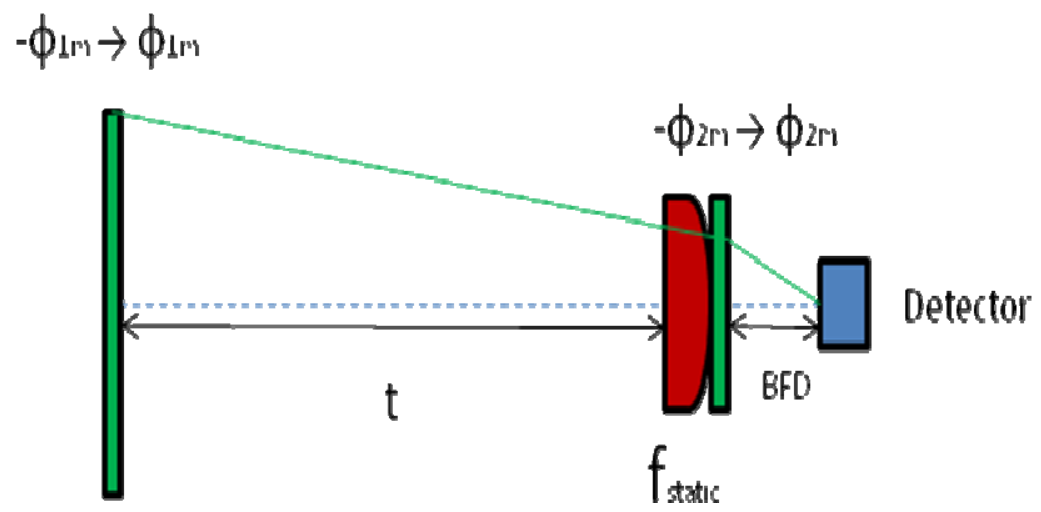

Figure 15: Schematic of an arbitrary zoom lens system.

Based on the desired zoom ratio and the maximum available power for the first (front) variable lens, the lens spacing and the required power range for the second variable lens (in the back) are determined. The desired Back Focal Distance (BFD) will dictate the focal length of the static lens and the focal length range of the overall system. This procedure is summarized in the following steps and equations: 


$$
\begin{aligned}
& \text { Zoom Ratio }=\frac{1+\phi_{1 \mathrm{~m}} \mathrm{t}}{1-\phi_{1 \mathrm{~m}} \mathrm{t}} \rightarrow \mathrm{t} \text { is determined } \\
& \left.\Delta \phi_{2}[\mathrm{t}]\right]:=1000 \frac{\frac{\phi_{1 \mathrm{~m}}}{\mathrm{R}[\mathrm{t}]}+\phi_{1 \mathrm{~m}}}{1-\phi_{1 \mathrm{~m}} \mathrm{t}} \rightarrow \Delta \phi_{2} \text { is determined } \\
& \mathrm{f}_{\text {static }}=\mathrm{f}_{2 \max }[\mathrm{BFD}]:=\frac{\left(1-t_{\circ} \phi_{1 \mathrm{~m}}\right) \text { BFD }}{1-\phi_{1 \mathrm{~m}}\left(\mathrm{t}_{\circ}+\mathrm{BFD}\right)} \rightarrow \mathrm{f}_{\text {static }} \text { and BFD are determined } \\
& \text { Focal length range: } \quad \Delta \mathrm{f}=\mathrm{t}_{\mathrm{O}} \mathrm{BFD} \Delta \phi_{2}\left[\mathrm{t}_{\mathrm{o}}\right]
\end{aligned}
$$

A Mathematica code was written to do the calculations using various parameters for the diffractive lenses. One example of this code is attached in Appendix H.

Our sixth and last paper "Adjustable Hybrid Diffractive/Refractive Achromatic Lens" attached in Appendix F addresses the correction of our diffractive lenses chromatic aberration by means of an adjustable pressure-controlled fluidic lens. The fluidic lens has a positive dispersion (opposite to the diffractive lenses) and can provide a more continuous variation. Therefore focal lengths of the fluidic lens are chosen such that they correct for the dispersion of the diffractive lens. We chose Methanol for the fluidic lens due to its high dispersion properties. The lenses showed good optical properties and the test results were close to the theoretical predictions as evident from the results presented in Appendix F. This paper is under review at the moment and in intended for the Optics Express journal of the Optical Society of America.

Diffractive lenses have a strong inherent chromatic aberration because the focal length of a diffractive lens has a strong dependence on wavelength given by $f=r_{I}{ }^{2} /(2 \lambda)$ where $r_{l}$ is the radius of the first Fresnel zone and $\lambda$ is the wavelength of the incident light. The focal length at any other wavelength $\lambda$, in $\mathrm{nm}$, can be scaled by the design 
wavelength according to $f(\lambda)=(555 / \lambda) f_{d}$ where $f_{d}$ is the design focal length at $555 \mathrm{~nm}$. Diffractive lenses have an Abbe number much smaller than their refractive counterparts. The Abbe number of a diffractive lens is unique in that it is equal to -3.45 , solely dependent upon the specified Fraunhofer $d, F, C$ wavelengths, as given by $\mathrm{V}_{\text {diffractive }}=\lambda_{d} /$ $\left(\lambda_{F}-\lambda_{C}\right)$. An approach was established to correlate the diffractive lens with the refractive lens. For certain focal lengths of the diffractive lens, $f_{d}$, we may choose the focal lengths and Abbe number of the fluidic lens such that they satisfy the achromat equation: $f_{f} V_{f}-3.45 f_{d}=0$, where $f_{f}$ and $V_{f}$ are the focal length and Abbe number of the fluidic lens, respectively. Refractive lenses have an Abbe number that is related to the indices of refraction as specified by the Fraunhofer $d, C, F$ lines where $\mathrm{V}_{\text {fluidic }}=\left(n_{d}-1\right) /\left(n_{F}-n_{C}\right)$. By knowing the focal length of the diffractive lens and the Abbe numbers of the refractive and diffractive lenses, one is able to determine the focal length range of the fluidic lens required to achieve an achromat.

For the future work, I suggest trying other types liquid crystal (specially the Polymer-Dispersed Liquid Crystals or the like that can work with any randomly polarized light) or even explore new electro-optic materials and find a way to reduce their operating voltages such as by doping them. It would be worthwhile to improve the diffractive pattern either by employing larger number of binary phase level Fresnel zones (such as $24,32,48$, etc) for higher overall diffraction efficiencies and better focal length tenability. Exploring other structures such as pixilated Spatial Light Modulators (SLMs) would also have a great potential. 


\section{REFERENCES}

1. H. Ren, S. Xu, Y.J. Lin, and S.T. Wu, "Adaptive-Focus Lenses," Optics \& Photonics News 19, 42-47 (2008).

2. C. A. López, C. Lee, and A. H. Hirsa, "Electrochemically activated adaptive liquid lens," Appl. Phys. Lett. 87, 134102 (2005).

3. M. Hain, R. Glöckner, S. Bhattacharya, D. Dias, S. Stankovic, and T. Tschudi, "Fast switching liquid crystal lenses for a dual focus digital versatile disc pickup", Opt. Comm., 188, 291-299, (2001).

4. H. Ren, Y.H. Fan, and S.T. Wu, "Tunable Fresnel lens using nanoscale polymerdispersed liquid crystals,” Appl. Phys. Lett. 83, 1515-1517 (2003).

5. S. Sato, A. Sugiyama and R. Sato, "Variable-Focus Liquid-Crystal Fresnel Lens," Jpn. J. Appl. Phys. 24 pp. L626-628 (1985)

6. S.C. Park, S.H. Lee, and J.G. Kim, "Compact Zoom Lens Design for a 5x Mobile Camera Using Prism," J. Opt. Soc. Korea 13, 206-212 (2009).

7. J.H. Sun, B.R. Hsueh, Y.C. Fang, J. MacDonald, and C.C. Hu, "Optical design and multiobjective optimization of miniature zoom optics with liquid lens element," Appl. Opt. 48, 1741-1757 (2009).

8. S. Kuiper, B.H.W. Hendriks, J.F. Suijver, S. Deladi, and I. Helwegen, "Zoom camera based on liquid lenses," Proc. SPIE, 6466, 64660F-1 (2007).

9. F. C. Wippermann, P. Schreiber, A. Bruer, and P. Craen, "Bifocal liquid lens zoom objective for mobile phone applications," Proc. SPIE 6501, 650109 (2007).

10. R. Peng, J. Chen, and S. Zhuang, "Electrowetting-actuated zoom lens with spherical-interface liquid lenses," J. Opt. Soc. Am. A 25, 2644-2650 (2008).

11. D. Y. Zhang, N. Justis, and Y. H. Lo, "Fluidic adaptive zoom lens with high zoom ratio and widely tunable field of view," Opt. Commun. 249, 175-182 (2005). 
APPENDIX A

LARGE-APERTURE SWITCHABLE THIN DIFFRACTIVE LENS WITH

INTERLEAVED ELECTRODE PATTERNS

APPLIED PHYSICS LETTERS 89, 141120 (2006)

\title{
Large-aperture switchable thin diffractive lens with interleaved electrode patterns
}

\begin{abstract}
Guoqiang Li, ${ }^{\text {) }}$ Pouria Valley, M. S. Giridhar, David L. Mathine, Gerald Meredith, Joshua N. Haddock, ${ }^{\text {b) }}$ Bernard Kippelen, ${ }^{\text {b) }}$ and N. Peyghambarian

College of Optical Sciences, University of Arizona, Tucson, Arizona 8572

(Received 12 April 2006; accepted 3 July 2006; published online 5 October 2006)

The authors report on a high-performance large-aperture switchable diffractive lens using nematic liquid crystal that can be used as an adaptive eyewear. The odd- and even-numbered ring electrodes are separated in two layers, avoiding the gaps between the neighboring electrodes and allowing high diffraction efficiency. It is easier to avoid shorts between neighboring conductive electrodes and fabricate lenses with larger aperture and smaller feature size. With a four-level phase modulation, a $15 \mathrm{~mm}$ aperture, $2 \mathrm{dpt}$ lens with small aberrations and diffraction efficiency of above $75 \%$ could be demonstrated with low operating voltages. The thickness of the liquid crystal is only $5 \mu \mathrm{m}$. The lens switching time is about $180 \mathrm{~ms}$. The on and off states of the electrically controlled lens allows near and distance vision, respectively. The focusing power of the lens can be adjusted to be either positive or negative. This structure can be extended to higher-level phase modulation with even higher efficiencies. 2006 American Institute of Physics. [DOI: 10.1063/1.2338646]
\end{abstract}

Spectacle correction of age-related optical changes in the eye has been increasingly important. With aging, the eye's lens loses some of its elasticity and becomes less able to focus incoming light. The result is that the eye has difficulty in switching easily between focusing on a near object and a distant object, a condition called presbyopia. To correct for this condition, various eyeglasses, including bifocal, trifocal, or progressive, or contact lenses have been developed to enable the eye to focus on both near and distant objects by looking through a different section of the lens, an approach referred to as area division. With the exception of the bifocal diffractive lens, ${ }^{1}$ the field of view for each type of vision is generally limited to a narrow corridor. Another choice is to use monovision lenses by which different focusing power is provided to each eye, one for near and the other for distant objects. However, in this case the binocular depth perception is affected. Ophthalmic lenses will be more capable and attractive if one could change their focusing power. ${ }^{2-4} \mathrm{Al}-$ though fluidic adaptive lenses have been demonstrated, ${ }^{5}$ an electrically controllable focusing lens is more suitable for this application. An electroactive lens allows for a greater field of view with the entire aperture and the focal length to be voltage controlled without mechanical motion. Different structures for liquid crystal (LC) adaptive lenses have been suggested for various applications, e.g., by filling empty lens shaped cavities with LC (Refs. 6 and 7) or by sandwiching $\mathrm{LC}$ between planar electrode plates ${ }^{8-21}$ and generating a refractive index gradient. The latter permits a power-failuresafe configuration for driving and thinner LC layers with fast switching, both of which are critical for ophthalmic lenses. However, the apertures of those lenses are not large enough, or high working voltages are required, or the LC layer is still relatively thick. The Fresnel zone structure allows relatively large aperture. A few binary Fresnel zone plates ${ }^{22-24}$ using $\mathrm{LC}$ as the active material have been demonstrated for imaging applications, but they did not provide viable solutions for

"Electronic mail: gli@optics.arizona.edu

Present address: School of Electrical and Computer Engineering, Georgia Institute of Technology, Atlanta, GA 30332. adaptive ophthalmic lenses. ${ }^{25}$ Therefore, it is important to investigate the approach that allows large aperture, fast response time, low operating voltages, high diffraction efficiency, and power-failure-safe configuration. In this letter, we present a design for high-efficiency switchable diffractive lens by separating odd- and even-numbered ring electrodes into two layers and thus eliminating the gaps between the neighboring electrodes. Compared to the design with the one-layer electrode pattern, another advantage of this concept is that it is easier to overcome shorts between neighboring conductive electrodes and fabricate lenses with larger aperture and smaller feature size.

The function of the diffractive lens is based on diffraction by a Fresnel zone pattern. ${ }^{26}$ By removing the multiple $2 \pi$ phase retardation from the refractive lens, a diffractive lens is obtained as shown in Fig. 1(a). The phase jump at each zone boundary is $2 \pi$ for the design wavelength $\lambda_{0}$, and the blazing profile in each zone provides for perfect constructive interference at the focal point. Figure 1(a) shows a four-level approximation (solid line) of the desired phase profile (dashed line). The structure is periodic in $r^{2}$ ( $r$ is the radius), and the period equals to $r_{1}^{2}$, where $r_{1}$ is the radius of the first zone. Each zone (subzone) has the same area as $r_{1}^{2}\left(r_{1}^{2} / N, N\right.$ is the discrete phase levels). The focal length of the diffractive lens is $f=r_{1}^{2} / 2 \lambda$. The diffraction efficiency of a multilevel diffractive lens is given by $\eta=\operatorname{sinc}^{2}(1 / N)$. Here we consider the nematic LC lens [Fig. 1(b)], where the phase profile is obtained by the electrically controlled birefringence effect. A nematic LC layer (E7 from Merck with birefringence greater than 0.2 ) is sandwiched between a patterned electrode substrate and a ground electrode substrate. The patterned electrode are fabricated by photolithographic processing of an indium tin oxide (ITO) film deposited on a glass substrate and consist of a circular array of rings. Both of the two electrode surfaces are coated with polyvinyalcohol as an alignment layer and are treated by rubbing to give a homogeneous molecular orientation. The refractive index experienced by the extraordinary beam is changed due to the reorientation of the LC molecule when a voltage is applied to the medium. The phase profile across 
141120-2 Li et al.

(a)
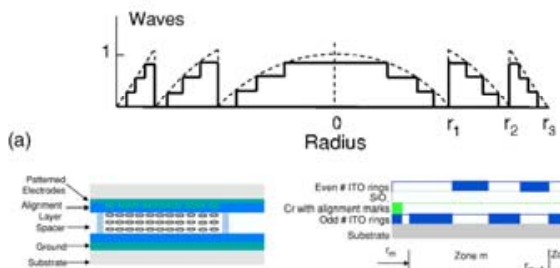

(b)
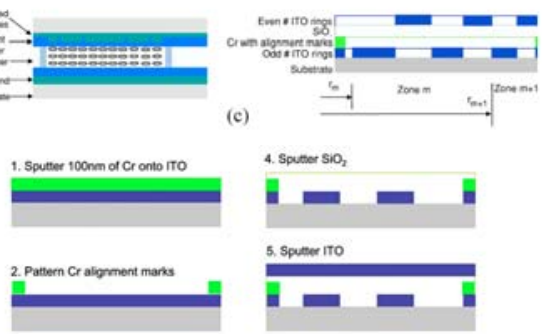

(d)

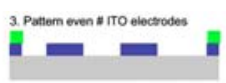

5. Sputeer ito
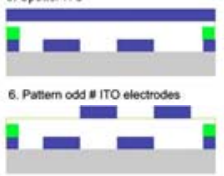

EG, 1. (Color online) Switchable LC diffractive lens. (a) Phase profile of a diffractive lens. Dashed line, continuous quadratic blaze profile; solid line, four-level digitized phase profile. (b) Structure of the flat LC lens. (c) Structure of the two-layer electrode pattern. (d) Fabrication procedure for the interleaved electrode pattern.

the lens is tailored by applying proper voltages to the patterned electrodes and as such, determines the diffraction efficiency. The phase profile may be affected by various factors, including the quantization error (number of phase levels in each zone), the gaps between the electrodes, fringing field effects in the transition area of neighboring zones, and fabrication errors. The quantization error can be reduced by increasing the number of phase levels in each zone. For instance, an ideal diffractive lens with two- and four-level digitization corresponds to efficiencies of $40.5 \%$ and $81.1 \%$, respectively. Simulations show that the gaps between the electrodes and different types of phase distortion at the electrode boundaries greatly affect diffraction efficiency and other performance parameters. To alleviate this effect, the odd- and even-numbered rings can be interleaved into two layers that are separated by an insulating layer such as $\mathrm{SiO}_{2}$. In this demonstration, all the subzones having the same counting index $n(n=1,2, \ldots, N)$ are connected together by a bus line, i.e., applied the same voltage, and thus have the same phase value. Figure $1(\mathrm{c})$ shows the cross section of the two-layer electrode pattern, where odd- and even-numbered rings are distributed in two layers and there are no gaps between two neighboring electrodes. The fabrication procedure for the interleaved electrode pattern is illustrated in Fig. 1(d). With this design, it is easier to avoid shorts between neighboring ITO electrodes and fabricate lenses with larger aperture and smaller subzones.

Here, we demonstrate a four-level, $15 \mathrm{~mm}$ aperture lens with a $2 \mathrm{dpt}$ focusing power at $555 \mathrm{~nm}$, the peak of the human photopic vision response. The width of the last subzone at the edge is $9.2 \mu \mathrm{m}$. After the lens is fabricated, various optical characterizations are performed. In the off state, transmission of the lens over the visible spectrum is above $85 \%$ if there is no antireflection coating on the surfaces. A polarized microscope is used to check the electro-optic function of each electrode. The lens is placed between two
Appl. Phys. Lett. 89, 141120 (2006)

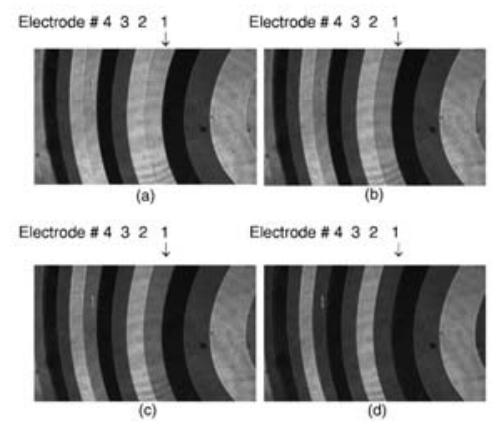

FIG. 2. Operation of the electrodes. Electrodes 2, 3, and 4 are set to certain voltages, respectively, while the voltage applied to electrode 1 was adjusted.

crossed polarizers, which are aligned with their transmission axes at $45^{\circ}$ and $-45^{\circ}$, respectively, to the horizontal axis. For each position, the intensity at the detector changes with the change of the phase difference $(\phi)$ between the ordinary and extraordinary components at the exit surface of the lens. The operation of each electrode can be inspected by observing the intensity variations over the area of said electrode. Figure 2 shows the intensity variation on electrode 1 when electrodes 2,3 , and 4 were set to certain voltages, respectively, and the voltage applied to electrode 1 was adjusted. The electrode boundaries can be clearly observed in the images.

To determine the diffraction efficiency as a function of lens area, the efficiency is measured for various beam sizes [Fig. 3(a)]. The efficiency for the $15 \mathrm{~mm}$ diameter area is above $75 \%$. As we expect, the lens without gaps has only a little decrease in efficiency when the activated area increases. The decrease is because the phase distortion caused by the fringing field has more significant effect on the outer zones. The efficiency of the center area is close to the theoretical value as the fringing field effect is negligible in this area. Dependence of the diffraction efficiency on the incidence angle is related to the field of view effect for normal use of

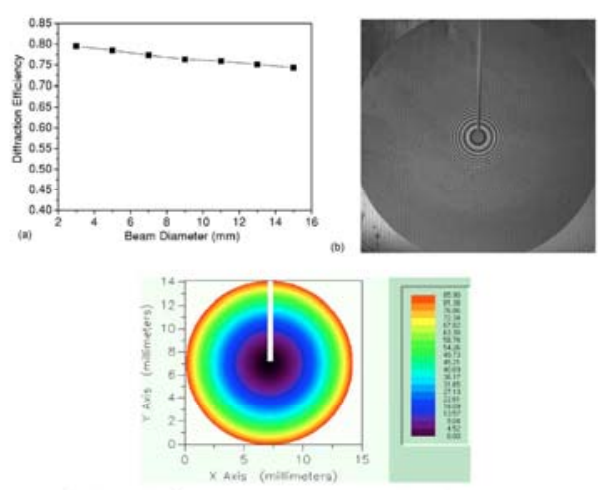

FIG. 3. (Color online) Some characterization results of the $15 \mathrm{~mm}$ aperture, four-level, 2 dpt lens, (a) Diffraction efficiency as a function of the beam diameter. (b) Interferogram obtained with the Mach-Zehnder interferometer. (c) Unwrapped phase map for a $14 \mathrm{~mm}$ aperture.

Downloaded 03 Nov 2006 to 150.135.248.49. Redistribution subject to AlP license or copyright, see http:l/apl.aip.org/apl/copyright.jsp 

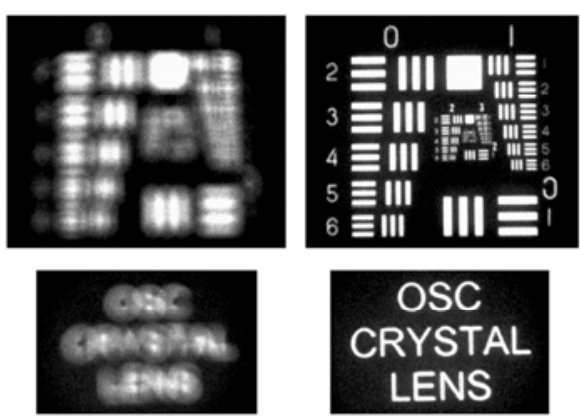

(a)

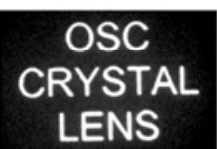

(b)

FIG. 4. Imaging using the 2 dpt electroactive diffractive lens with the model eye. The object is placed at a reading distance $(\sim 30 \mathrm{~cm})$. (a) The image is severely out of focus in the model eye when the diffractive lens is OFF. (b) When the diffractive lens is activated, the object is imaged clearly.

the spectacle lenses. From the experiment, the diffraction efficiency decreases monotonically as the increase of the incidence angle. It drops about $4 \%$ when the lens is tilted $20^{\circ}$ about the lateral axis.

A Mach-Zehnder interferometer operating at $543.5 \mathrm{~nm}$ was used to measure wave-front quality immediately behind the lens and to determine the focal length. The technique is based on interference between the spherical wave after the lens and a reference plane wave. Figure 3(b) shows an example of the measured interferograms. Five interferograms were taken with a phase shift between each interferogram and the wrapped phase map can be produced. The unwrapped phase map represents the actual optical path difference profile generated by the diffractive lens is shown in Fig. 3(c). A good spherical wave was obtained with very few higher order aberrations as indicated by a rms wave-front error of $0.0889 \lambda$. The focal length was found to be $50.855 \mathrm{~cm}$, the focal length at $555 \mathrm{~nm}$ was calculated as $49.80 \mathrm{~cm}$ which corresponds to a focusing power of $2.008 \mathrm{dpt}$. The focused spot size was also measured and found to be $47.9 \mu \mathrm{m}$, close to the diffraction-limited spot size of $45.1 \mu \mathrm{m}$. The response time and the decay time were measured to be 180 and $120 \mathrm{~ms}$, respectively. All these parameters indicate high performance of the lens. In addition, we also verified that, by changing the slope of the applied voltages to each zone, a negative $2 \mathrm{dpt}$ focusing power can be obtained with the same lens, and the diffraction efficiency is the same as the positive 2 dpt case.

As nematic liquid crystal is polarization sensitive, two LC lenses with orthogonal alignment directions are required to form a complete lens that works for randomly polarized light. The double-element complete lens has been demonstrated to provide near vision correction for a accommodation-free model eye consisting of a $60 \mathrm{dpt}$ refractive lens, an iris, and a charge-coupled device array. A white light source illuminates the object placed at a typical reading distance of approximately $30 \mathrm{~cm}$. When the diffractive lens is not activated, the image is blurred [Fig. 4(a)], but when the diffractive lens is turned on, the object is imaged clearly [Fig. 4(b)]. The on and off states of the lens allow near and distance vision, respectively.
In conclusion, we have demonstrated a highperformance switchable electro-optic adaptive diffractive lens for vision correction of presbyopia. By interleaving the odd-numbered and even-numbered electrodes into two layers, there is no gap between the neighboring electrodes. This helps to maintain the desired diffraction efficiency and it makes it easier to fabricate lenses with large aperture and small feature size without shorts between the ring electrodes. Diffraction efficiency of above $75 \%$ has been achieved for four-level lenses with $15 \mathrm{~mm}$ aperture. This design methodology can be extended to phase steps larger than 4 , where the electrodes can be addressed from an additional layer through vias. However, more fabrication steps are required. The focusing power of the lens can be adjusted to be either positive or negative, depending on the voltages applied to the patterned electrodes. Other advantages of this lens include compactness, lighter weight, low cost, and easier operation with low voltages and low power dissipation. For people who also need correction for distance vision, an additional refractive lens can be integrated with the switchable diffractive lens. Using nematic LC, two LC lenses set in orthogonal direction are required to make it polarization insensitive. In order to avoid light loss due to back reflections at the substrate interfaces, antireflection coating is necessary.

This work was supported in part by the Technology and Research Initiative Fund program of the State of Arizona.

${ }^{1} J$. A. Futhey, Proc. SPIE 1052, 142 (1989).

G. Smith and D. A. Atchison, The Eye and Visual Optical Instruments (Cambridge University Press, New York, 1997).

'G. Vdovin, M. Loktev, and A. Naumov, Opt. Fxpress 11, 810 (2003).

${ }^{4}$ A. W. Lohmann, Appl. Opt. 9, 1669 (1970); H. J. Caulfield, Opt. Laser Technol. 34. 1 (2002).

34, 1 (2002). Op. 9. I669 (1970); H. J. Cand

D.-Y. Thang, V. Lien, Y. Berdichevsky, J. Choi, and Y.-H. Lo, Appl. Phys. Lett. 82, $3171(2003)$

${ }^{6}$ S. Sato, A. Sugiyama, and R. Sato, Jpn. J. Appl. Phys., Part 2 24, L626 (1985).

'L. G. Commander, S. E. Day, and D. R. Selviah, Opt. Commun. 177, 157 (2000).

${ }^{8}$ S. T. Kowel, D. S. Cleverly, and P. G. Kornreich, Appl. Opt. 23, 278 (1984).

A. Nouhi and S. T. Kowel, Appl. Opt. 23, 2774 (1984).

${ }^{10}$ A. F. Naumov, M. Yu. Loktev, I. R. Guralnik, and G. Vdovin, Opt. Lett. 23, 992 (1998)

"M. Y. Loktev, V. N. Belopukhov, F. L. Vladimirov, G. V. Vdovin, G. D, Love, and A. F. Naumov, Rev, Sci. Instrum. 71, 3190 (2000).

${ }_{12} \mathrm{~N}$. A. Riza and M. C. Delule, Opt. Lett. 19, $1013(1994)$.

${ }^{13}$ P. W. McOwan. M. S. Gordon, and W. J. Hossack, Opt. Commun. 103, 189 (1993)

14 B. Wang, M. Ye, and S. Sato, Appl. Opt. 43, 3420 (2004).

is W. Klaus, M. Ide, Y. Hayano, S. Morokawa, and Y. Arimoto, Proc. SPIE 3635, 66 (1999).

Y. Sun, G. P. Nordin, S. T. Kowel, and B. Wang, Proc. SPIE 4987, 209 (2003).

${ }^{17}$ H. Ren and S.-T. Wu, Appl. Phys. Lett. 81, 3537 (2002).

${ }^{18}$ H.-S. Ji, J.-H. Kim, and S. Kumar, Opt. Lett. 28, 1147 (2003).

${ }^{19} \mathrm{~V}$. V. Presnyakov and T. V. Galstian, J. Appl. Phys. 97, 103101 (2005).

${ }^{20}$ T. Nose, S. Masuda, S. Sato, J. Li, L. Chien, and P. J. Bos, Opt. Lett. 22 . 351 (1997).

${ }^{21}$ W. N. Charman, Ophthalmic Physiol. Opt. 13, 427 (1993).

${ }^{22}$ W. W. Williams. N. J. Powell, and A. Purvis, Proc. SPIE 1168. 352 (1989).

${ }^{22}$ G. Williams, N. J. Powell, and A. Purvis, Proc. SPIE

${ }^{23}$ J. S. Patel and K. Rastani, Opt. Lett. 16, 532 (1991).

${ }^{24}$ H. Ren, Y.-H. Fan, and S.-T. Wu, Appl. Phys. Lett. 83, 1515 (2003).

${ }^{25}$ C. W. Fowler and E. S. Pateras, Ophthalmic Physiol. Opt. 10, 186 (1990).

${ }^{26} \mathrm{D}$. W. Prather, M. S. Mirotznik, and S. Shi, Mathematical Modeling in Optical Sciences (SIAM, Philadelphia, 2000); S. Sinzinger and J. Jahns, Microoptics (Wiley-VCH, Cambridge, 2003). 


\title{
SWITCHABLE ELECTRO-OPTIC DIFFRACTIVE LENS WITH HIGH EFFICIENCY
}

\author{
FOR OPHTHALMIC APPLICATIONS
}

\section{Switchable electro-optic diffractive lens with high efficiency for ophthalmic applications}

\begin{abstract}
Guoqiang Li"*, David L. Mathine*, Pouria Valley*, Pekka Äyräs", Joshua N. Haddock, M. S. Giridhar*, Gregory Williby*, Jim Schwiegerling $\star \S$, Gerald R. Meredith*, Bernard Kippelen ${ }^{\ddagger}$, Seppo Honkanen*, and Nasser Peyghambarian*t

*College of Optical Sciences, University of Arizona, Tucson, AZ 85721; „school of Electrical and Com puter Engineering, Georgia Institute of Technology, Atlanta, GA 30332; and śDepartm ent of Ophthalmology and Vision Sciences, University of Arizona, Tucson, AZ 85711
\end{abstract}

Communicated by Nicolaas Bloembergen, Univer sity of Arizona, Tucson, AZ, February 2, 2006 (received for review December 10, 2005)

Presbyopia is an age-related loss of accommodation of the human eye that manifests itself as inability to shift focus from distant to near objects. Assuming no refractive error, presbyopes have clear vision of distant objects; they require reading glasses for viewing near objects. Area-divided bifocal lenses are one example of treatment for this problem. However, the field of view is limited in such eyeglasses, requiring the user to gaze down to accomplish near-vision tasks and in some cases causing dizziness and discomfort. Here, we report on previously undescribed switchable, flat liquid-crystal diffractive lenses that can adaptively change their focusing power. The operation of these spectacle lenses is based on electrical control of the refractive index of a $5-\mu \mathrm{m}$-thick layer of nematic liquid crystal using a circular array of photolithographically defined transparent electrodes. It operates with high transmission, low voltage $(<2 \mathrm{~V}$ ) fast response $(<1$ sec), diffraction isficicion, efficiency $>90 \%$, small aberrations, and a power-failure-safe configuration. These results represent significant advance in state of-the-art liquid-crystal diffr active lenses for vision care and other
applications. They have the potential of revolutionizing the field of presbyopia correction when combined with automatic adjustable focusing power.

ophthalmic lens | switchable lens | vision correction

The use of nematic liquid crystals to implement switchable lenses has been proposed previously but had limited success for ophthalmic applications (1). Hybrid liquid-crystal refractive lenses incorporating convex and concave substrates have been demonstrated $(2,3)$. However, the large thickness of the liquid crystal layers $(>400 \mu \mathrm{m})$ make their response and recovery times long and their transmission low because of optical scattering. To reduce the thickness of the active layer, surface relief Fresne lens substrates have been proposed (4). However, in this geometry the lens is optically active in the electrically off-state, which is not desirable for ophthalmic applications where a loss of electrical power could suddenly result in near-vision correction during a critical distance vision task such as driving. In other approaches, thin uniform layers of liquid crystal were used, and refractive lenses were produced by the use of discrete electrodes (5) continuous highly resistive electrodes (6) or spatially dis(5), cont ectric fields (microlenses) (7). Hower, in these lenses either the range of focal length or their small diamete made them unsuitable for ophthalmic applications. We employ a photolithographically pattemed thin diffractive lens with large aperture, fast response time, and a power-failure-safe configuration to overcome these limitations. Although high-efficiency liquid-cryst al-based diffractive devices have been demonstrated for beam-steering $(8,9)$, less effort was given to the development of switchable diffractive lenses. The diffraction efficiencies of the lenses achieved for iming $(10-15)$ and other applica the lenses achieved for inging (10-15) and other application $(16,17)$ were too low to meet the requirements of ophthalmic applications.

Fig 1 a compares the shape (phase profile) of a refractive lens (dashed line) with an ideal diffractive lens (dotted line). The diffractive lens is produced by removing the multiple $2 \pi$-phase ret ardation from the refractive lens, resulting in multiple Fresnel zones. The phase jump at each zone boundary is $2 \pi$ for the design wavelength. The outer radius of each zone is given by (18)

$$
r_{m}=\sqrt{2 m \lambda f}, \quad m=1,2, \ldots, M
$$

where $m$ is a counting index that refers to successive Fresnel zone starting in the center, $\lambda$ is the wavelength, and $f$ is the focal length. To digitize the process, the continuous phase profile in each zone is divided into multiple subzones with a series of each zone is divided into multiple subzones with a series of
discrete phase levels (19) ("staircase" structure; Fig. 1a). The outer radius of each subzone is given by

$$
r_{m, n}=\sqrt{2[(m-1)+n / L] \lambda f}, \quad n=1,2, \ldots, L,
$$

where $L$ is the number of phase levels per zone, and $n$ is the counting index of the individual phase levels. Diffraction efficiency increases by increasing the number of subzones $L$, reach ing maximum values of $40.5 \%, 81.1 \%$, and $95.0 \%$ for lenses with two, four, and eight phase levels per zone, respectively.

Diffractive lenses with eight subzones, $10-\mathrm{mm}$ diameters, and focal lengths of 1 and $0.5 \mathrm{~m}(+1.0$ and +2 diopter of add power, respectively) were demonstrated at the peak of the human photopic response, $555 \mathrm{~nm}$. The schematic drawings of the electrode pattem and the fabrication procedure are shown in Fig. $1 b$ and $c$, respectively. Using photolithographic techniques, concentric and rotationally symmetric transparent indium tin oxide electrodes ( $50 \mathrm{~nm}$ in thickness), whose radii were determined by Eq. 2, were patterned on a float-glass substrate. A $1-\mu \mathrm{m}$ gap was required between adjacent electrodes to maint ain electrical isolation and ensure a smooth transition of the phase electrical isolation and ensure a smooth transition of the phase
profile introduced by the liquid crystal. Over the patterned indium tin oxide, a 200 -nm-thick electrically insulating layer of $\mathrm{SiO}_{2}$ is sputtered and into which small via openings $(3 \times 3 \mu \mathrm{m})$ were etched, allowing electrical contact to be made to the underlying electrodes. An electrically conductive layer of $\mathrm{Al}$ is subsequently sputtered over the insulating layer to fill the vias pendent electrical bus bars ( 6 - $\mu \mathrm{m}$ wide within the lens). Each bus bar connects the discrete phase level electrodes of equal counting index $n$ in all Fresnel zones (as shown in Fig. 1b) such that only eight external electrical connections (plus one ground connection) are required per lens.

The patterned substrate, as well as an additional substrate with a continuous indium tin oxide electrode that acts as the electrical ground, were spin coated with poly(vinyl alcohol) to act as liquid-cryst al alignment layer. The alignment layers were rubbed with a velvet cloth to achieve homogeneous alignment, and the two substrates were assembled. The commercial nematic liquid

Conflict of interest statement: No conflicts declared. Abbreviation: MTF, modulation transfer function.

To whom correspondence may be addressed. E-mail: glieoptics.arizona.edu or nnp@optics.arizona.edu.

(6) 2006 by The National A cademy of Sciences of the USA 
a

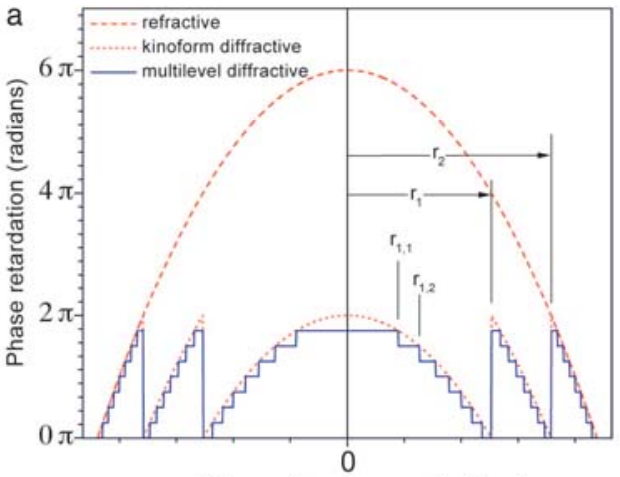

Distance from lens center (a.u.) $b$

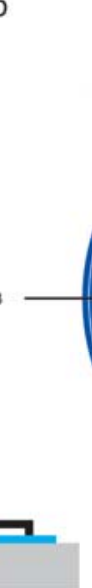

PVA
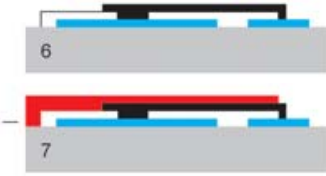

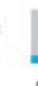

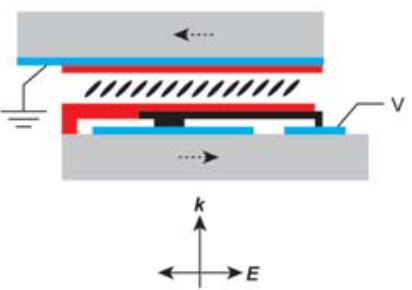

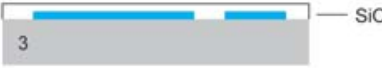
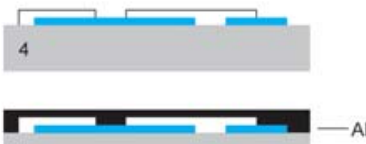

5

Fig. 1. Adaptive liquid-crystal diffractive lens. (a) Dashed line, phase profile of a conventional refractive lens; dotted line, phase profile to achieve a diffractive lens; staircase structure, multilevel quantization approximates the continuous quadratic blaze profile. a.u., arbitrary units. (b) Layout of the one-layer electrode pattern (two central zones shown). Adjacent zones are distinguished by color. An electrical insulation layer with vias is added (vias shown with white dots). Each bus connects to one electrode (subzone) in each zone. Dimensions of the vias, the bus line, and the gap between electrodes are illustrated in the lower right. (c) Processing steps for fabrication of the patterned electrodes and the conductive lines. The structure of the liquid-crystal lens is shown in the lower right, where $k$ is the wave vector, and $E$ is the polarization state of the incident light.

crystal E7 (Merck) was used as the electro-optic medium and was filled by capillary action into the empty cell at a temperature above the clearing point $\left(60^{\circ} \mathrm{C}\right)$ and then cooled at $1^{\circ} \mathrm{C} / \mathrm{min}$ to room temperature. The cell then was sealed with epoxy and connected to the drive electronics. The drive electronics consis of custom-fabricated integrated circuits that contain eight independently controlled output channels. Each channel generates a modified square waveform with variable peak-to-peak amplitude between 0 and $5 \mathrm{~V}$.

\section{Results}

The lens showed excellent performance (because of space limitations, we only describe the results for the 1-diopter lens) In the optically inactive state (voltage off) in which the lens has no focusing power, optical transmission is $85 \%$ over the visible spectrum, a value that can be increased by the use of ophthalmic quality substrates and antireflection coatings. Monochromatic $(543.5 \mathrm{~nm})$ polarized microscopy images of the lens under operation (see Methods) indicate that all eight electrode sets operated properly and provided discrete phase changes (Fig. 2a). Eight optimized drive voltages with amplitudes between 0 and 2 $V$ produced a maximum first-order diffraction efficiency of $91 \%$, near the $95 \%$ predicted by scalar diffraction theory. The measured diffraction efficiency as a function of lens area (see Methods) reaches $94 \%$ near the center of the lens, decreasing monotonically as the area is increased (Fig. $2 b$ ). The decrease is due to the fact that phase distortion caused by the fringing field at the zone boundaries has more significant effect at the outer zones as the width of each electrode becomes smaller. At the edges of the electrodes, the electric field lines are not perpendicular to the liquid-crystal lens substrate, and the fringing fields cause the phase transitions at the zone boundaries to be not as sharp as in the ideal case, thus inducing phase distortions and reducing the diffraction efficiencies $(20,21)$. The focused spot size is $\approx 135 \mu \mathrm{m}$, which is also close to the diffraction-limit value of $133 \mu \mathrm{m}$. The lens shows subsecond switching time.

Interferometric measurements at $543.5 \mathrm{~nm}$ (see Methods) show excellent imaging capability of the lens. Strong modulation 

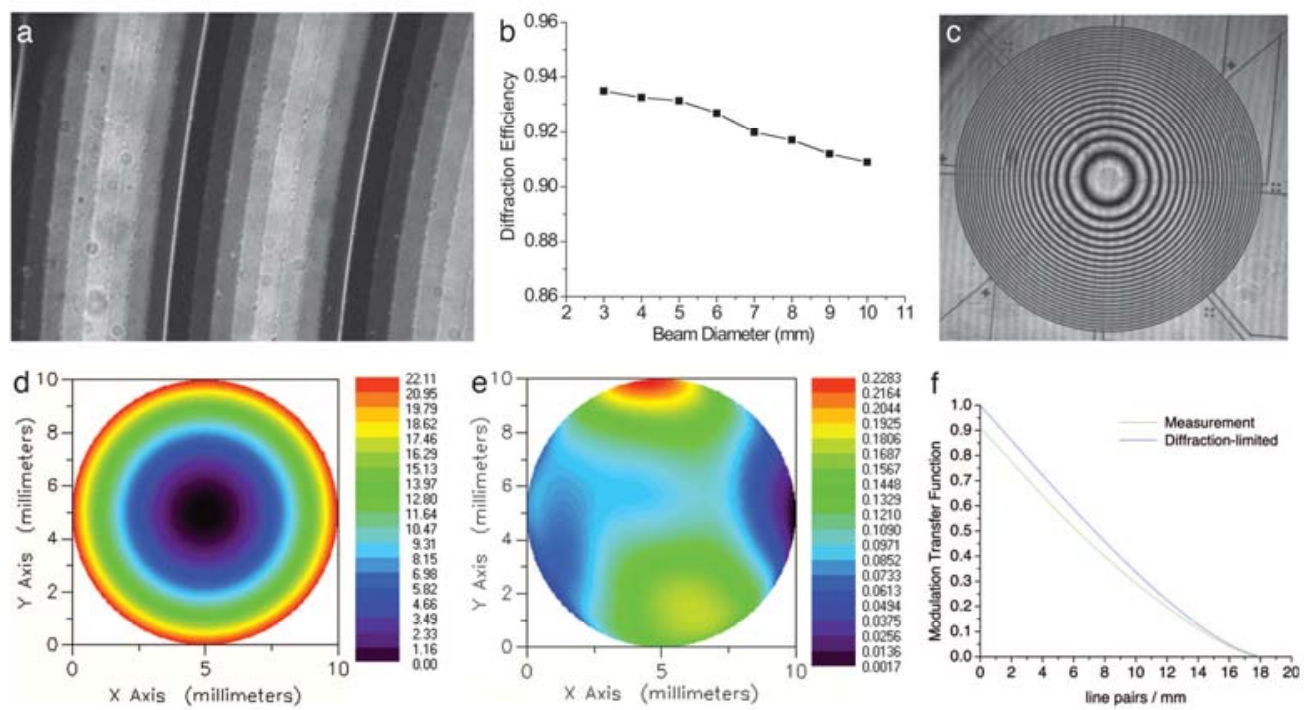

Fig. 2. Characterization of the 1-diopter lens. (a) Electro-optic response of the lens obtained with polar ized microscope. (b) Diffraction efficiency as a function of the beam diameter. (c) Interferogram obtained with the Mach-Zehnder interferometer. The interference pattern has very good fringe modulation across the lens. A close-up view of the interferogram shows that the eight subzones in each zone have different grayscale intensities, and the pattern is periodic. (d) Unwrapped phase map for a 10-mm aperture. (e) Phase map of the unwrapped phase minus tilting and focusing. ( $f$ ) MTF of the lens. The green line is obtained from the measurement data, and the blue line is for a diffraction-limited lens. The value at low spatial frequency is determined by the diffraction efficiency of the lens.

of the optical power is observed in interferogram of the lens in the optically active state (Fig. $2 c$ ). The unwrapped phase map of the lens is shown in Fig. $2 d$ with a peak-to-valley optical path length of $23.05 \lambda$. The focusing power was estimated to be 1.002 diopter, in excellent agreement with the design value. Very good spherical profiles were obtained in both $x$ and $y$ cross sections, indicating small aberrations. Higher-order aberrations were estimated by analyzing the difference between the measured wavefront and a best-fit spherical wave and tilt (Fig. 2e). The peak-to-valley range of the difference is $0.241 \lambda$, and the rms value is $0.039 \lambda$, which is comparable with a high-quality reading glass. The modulation transfer function (MTF) indicated near diffraction-limited performance (Fig. 2f). All properties of the lens, as shown in Fig. 2, make the switchable lens suitable for ophthalmic applications.

To test the imaging properties of the lens, a model human eye was constructed by using a fixed, +60 diopter achromatic doublet glass lens and a monochrome charge-coupled device (CCD) with a filter to match the human photopic response. Because homogeneously aligned nematic liquid crystals are polarization sensitive, two lenses with orthogonal alignment directions were used in series to create a single polarizationinsensitive lens. Two such lenses were aligned and cemented together. To simulate a typical near-vision task such as reading, a double-element lens was placed in front of the model eye and used to image a test object illuminated with unpolarized white light placed $30 \mathrm{~cm}$ in front of the lens. As can be seen in Fig. $3 a$, the model eye has insufficient power to form a sharp image, but by switching on the diffractive lens the image is brought into focus (Fig. $3 b$ ). The double-element lens has excellent optical transmission. To test the imaging performance of the lenses with actual human subjects, a pair of test spectacles has been con-

6102 | www.pnas.org/cgi/doi/10.1073/pnas.0600850103 structed (Fig. 4), and initial clinical results agree well with the model eye test. When the electro-optic lenses are both in the inactive state, there is no noticeable degradation in the quality of the distant vision. For chromatic aberration, an achromatic diffractive lens can be designed by introducing $p 2 \pi(p>1$, integer) phase jump at the zone boundaries for the design wavelength (22). In practice, the ocular lens itself has a chromatic aberration that is less than the diffractive lens. Assuming the brain is adapted to a certain degree of chromatic aberration, balancing the dispersion of the diffractive lens and the eye is less desirable. More clinical study needs to be performed on this aspect.

\section{Conclusion}

In conclusion, we have demonstrated switchable liquid-crystal diffractive lenses with high diffraction efficiency, high optical quality, rapid response time, and diffraction-limited performance. These flat lenses are highly promising to replace conventional area division refractive, multifocal spectacle lenses used by presbyopes. They have the potential of revolutionizing the field of presbyopia correction when it is combined with automatic adjustable focusing power. Negative focusing powers also can be achieved with the same lenses by changing the sign of the slope of the applied voltages. The use of these lenses is not limited to ophthalmology but can be extended to numerous other applications including dentistry where switchable lens elements with relatively large diameters are desirable.

\section{Methods}

When the device is fabricated, various optical characterizations are performed. 

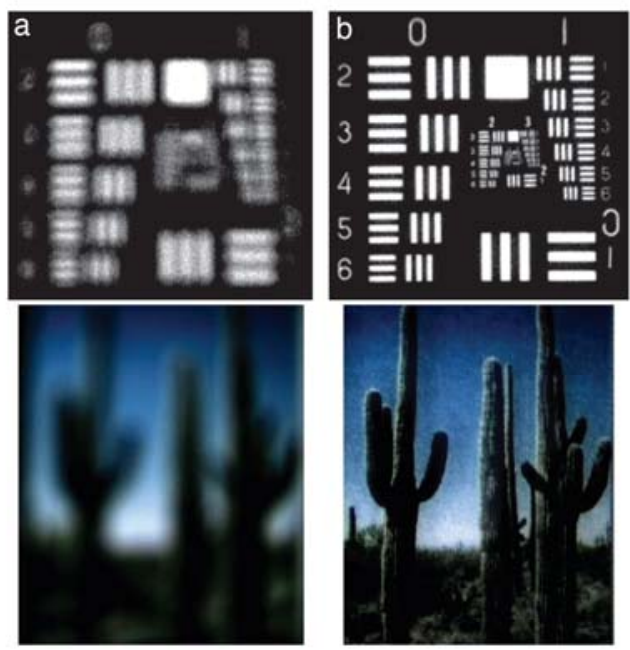

Fig. 3. Hybrid imaging using the 1-diopter electroactive diffractive lens wit the model eye. The function of the diffractive lens is to provide near-vision correction to the model eye. (a) The object is placed at a reading distance $(\sim 30$ $\mathrm{cm}$ ). The image is severely out of focus in the model eye when the diffractive lens is off. (b) When the diffractive lens is activated, the object is imaged clearly.

Polarized Microscopy. A computer-interfaced polarized optical microscope with a laser source at $543.5 \mathrm{~nm}$ was constructed on the optical bench and used to inspect the lenses on a microscopic scale and verify that all electrodes were operating properly. The lens was placed between crossed polarizers where the transmission axes were oriented at angles of $45^{\circ}$ and $-45^{\circ}$ with respect to the liquid-crystal alignment layer rub direction. For each position on the lens, the intensity seen by the charge-coupled device camera is a function of the voltagedependent phase retardation $\phi(V)$ between the ordinary and extraordinary wave components at the exit surface of the lens. The voltage dependent transmission between crossed polarizers $[T(V)]$ is given by

$$
T(V)=\sin ^{2}\left(\frac{\phi(V)}{2}\right),
$$

where the transmission is a maximum when $\phi(V)=\pi$ and a minimum when $\phi(V)=2 \pi(23)$. Therefore, the voltage-

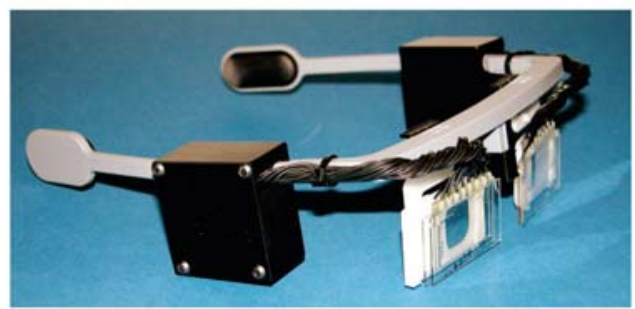

Fig. 4. A prototype of the assembled adaptive eyewear. dependent phase retardation of each electrode can be inspected by observing the intensity variations over its area.

Measurement of Diffraction Efficiency. Diffraction efficiency is the amount of light intensity that goes to a particular diffraction order compared with the sum of intensities in all of the diffraction orders. To determine the efficiency of the first diffraction orders. To determine the efficiency of the first diffracted order, a linearly polarized $543.5-\mathrm{nm}$ laser beam was
expanded to a diameter of $10 \mathrm{~mm}$ and allowed to pass through the active area of the lens. The power of the beam was measured in the focal plane with the lens inactive (total power) and then again with the lens activated (diffracted power). The ratio of diffracted power to total power is the diffraction efficiency. A small aperture in front of the detector was used to isolate the first-order light when the lens was activated, and as all measurements were made down-stream of the lens, they were automatically corrected for Fresnel losses. To measure the diffraction efficiency as a function of lens area, a variable aperture (positioned concentrically with respect to the lens) was placed in front of the diffractive lens to control the size of the beam. The diffraction efficiency then was measured as the diameter of the beam was increased from 3 to $10 \mathrm{~mm}$ in $1-\mathrm{mm}$ increments.

Interferometric Testing and MTF Calculation. The performance of the diffractive lenses also was evaluated by using a computerinterfaced, phase-shifting Mach-Zehnder interferometer with a linearly polarized $543.5-\mathrm{nm}$ laser source $(24,25)$. The lens under test was placed in the object arm of the interferometer and then imaged onto a charge-coupled device camera such that the captured interference patterns were formed by the converging wavefront generated at the exit face of the lens and the reference plane wave. A small aperture was placed between the imaging lens and the camera at the point of focus to isolate and test only the wavefront generated by the first diffracted order. Multiple $\pi / 2$ phase shifts were generated in the reference arm by using a piezoelectric transducer actuated mirror, and a phase unwrapping algorithm then was used to generate a phase map of the diffracted wavefront. From this unwrapped phase map of the wavefront immediately behind the diffractive lens, the focal length $(f)$ of the diffractive lens is calculated by using

$$
f=\frac{\rho^{2}}{2 O P D}
$$

where OPD is the peak-to-valley optical path difference from center to edge, and $\rho$ is the radius of the test area (26). Because the lenses were designed for operation at $555 \mathrm{~nm}$ but tested at $543.5 \mathrm{~nm}$, correction of the extracted focal length value was made by

$$
f(\lambda)=f_{0} \frac{\lambda_{0}}{\lambda},
$$

where $f_{0}$ and $\lambda_{0}$ are the design focal length and wavelength, respectively, and $\lambda$ is the measurement wavelength (27). Measurement of the peak-to-valley and rms errors in the wavefront were made subsequent to removing the best-fit sphere and any tilt from the phase map.

The imaging performance of the lens can be evaluated in terms of MTF, which represents the ratio of the image modulation to the object modulation at all frequencies. The wavefront of the first-order diffraction can be expressed by a sum of Zernike polynomials (28), and the MTF thus can be calculated by normalized autocorrelation of the generalized pupil function (26).

We thank the Technology and Research Inititative Fund program of the State of Arizona. 
1. Smith, G. \& Atchison, D. A. (1997) The Eye and Visual Optical Instruments (Cambridge Univ. Press, Cambridge, U.K.)

. Sato, S. (1985) Jpn. J. Appl. Phys. 18, 1679-1684.

Fowlet, C. W. \& Pateras, E. S. (1990) Ophthal. Physiol. Opt. 10, 186-194

4. Sato, S., Sugiyama, A. \& Sato, R. (1985) Jpn. J. Appl. Phys. 24, L626-L628.

Kowel, S. T., Clevetly, D. S. \& Kornteich, P. G. (1984) Appl. Opt. 23, 278-289. 6. Naumov, A. F., Loktev, M. Y., Gutaln k, I. R. \& Vdovin G. (1998) Opt. Leth 23, 992-994.

7. Masuda, S., Takahashi, S., Nose, T., Sato, S. \& Ito H. (1997) Appl. Opt. 36,

Friedman, L. J., Hobbs, D. S., Lieberman, S., Corkum, D. L., Nguyen, H. Q Reslet, D. P., Shatp, R. C. \& Dorschner, T. A. (1996) Appl. Opt. 35, 6236-6240. Reslet, D. P., Hobbs, D. S., Shatp, R. C., Friedman, L. J. \& Dotschner, T. A (1996) Opt. Lett. 21, 689-691.

10. Williams, G. Powell, N. J. \& Purvis, A. (1989) Proc. SPIE 1168, 352-357.

11. Patel, J. S. \& Rastani, K. (1991) Opt. Lett. 16, 532-534.

12. Dance, B. (1992) Laser Focus Word 28,34 .

13. Wiltshine, M. C. K (1993) GEC J. Res, 10, 119-125.

14. McOwan, P. W Gotdon, M. S. \& Hosact. W. J. (1993) Opt Commun 103, $189-193$.
15. Ren, H., Fan, Y.-H. \& Wu, S.-T. (2003) Appl. Phys. Lett. 83, 1515-1517 16. Laude, V. (1998) Opt. Comm 153, 134-152.

7. Hain, M., Glöcknet, R., Bhattachatya, S., Dias, D., Stankovic, S. \& Tschudi, T. (2001) Opt. Comm. 188, 291-299.

18. Kress, B. \& Mey, P. (2000) Digital Diffractive Optics (Wiley, New York).

19. Fatn, M. W. \& Veldkamp, W. B. (1994) in Handbook of Optics, ed. Bass, M. (McGraw-Hill, New Yotk), Vol. II, Chap. 8, p. 8.15.

20. Btinkley, P. F, Kowel, S. T. \& Chu, C. (1988) Appl. Opt. 27, 4578-4586.

21. Aptet, B., Efton, U. \& Bahat-Treidel, E. (2004) Appl. Opt. 43, 11-19.

22. Faklis, D. \& Motris, G. M. (1995) Appl. Opt. 34, 2462-2468

23. Wu, S. T., Efton, U. \& Hess, L. D. (1984) Appl. Opt. 23, 3911-3915,

24. Williby, G. (2003) Ph.D. dissertation (Optical Sciences Center, Univ. of Arizona, Tucson, AZ).

25. INTELLIWAVE (1997) (Engineering Synthesis Design, Inc., Tucson, AZ)

26. Botn, M. \& Wolf, E. (1999) Principles of Optics (Cambridge Univ. Press, Cambridge, U.K.), 7 th Ed.

. G. M. (1997) Appl. Opt. 36, 253-257.

J. C. \& Creath, K. (1992) in Applied Optics and Optical Engineering, eds. Shannon, R. R. \& Wyant, J. C. (Academic, London), Vol. XI, Chap. 1, p. 31 
APPENDIX C

HIGH-EFFICIENCY SWITCHABLE FLAT DIFFRACTIVE OPTHALMIC LENS

WITH THREE-LAYER ELECTRODE PATTERN AND TWO-LAYER VIA

STRUCTURE

APPLIED PHYSICS LETTERS 90, 111105 (2007)

\title{
High-efficiency switchable flat diffractive ophthalmic lens with three-layer electrode pattern and two-layer via structures
}

\begin{abstract}
Guogiang Li, ${ }^{\text {a) }}$ Pouria Valley, Pekka Äyräs, David L. Mathine, Seppo Honkanen, and N. Peyghambarian

College of Optical Sciences, University of Arizona, Tucson, Arizona 85721

(Received 2 January 2007; accepted 3 February 2007; published online 13 March 2007)

A general design for switchable, flat, liquid crystal diffractive lens with three-layer electrode pattern and two-layer via structures is reported for near-, intermediate-, and distance-vision corrections of presbyopic eyes. The microfabricated transparent concentric ring electrodes are distributed in two layers and different voltages are applied to each electrode through bus lines in another layer. Connection between the electrodes and the bus lines is achieved by vias (conducting holes for vertical interconnections) in the third dimension. For demonstration, a lens is first tuned with a focal length of $1 \mathrm{~m}$ ( 1 diopter add power), eight-level phase modulation, and diffraction efficiency above $92 \%$ and then reconfigured to operate as a 2 diopter four-level lens with a diffraction efficiency of $78 \%$. The lens operates with low voltages and a rise time of $150 \mathrm{~ms}$. This design methodology allows the aperture needed for practical use and a power-failure-safe configuration. $\odot 2007$ American Institute of Physics. [DOI: 10.1063/1.2712773]
\end{abstract}

Switchable liquid crystal (LC) lenses have attracted significant research interest. Various LC lenses ${ }^{1-6}$ with variable focus have been applied for optical imaging. interconnection, ${ }^{2}$ data reading in optical storage, ${ }^{3}$ and vision correction. ${ }^{4}$ They have advantages such as compactness, lightweight, and electrical control of focal length without mechanical movement. ${ }^{7,8}$ Nematic LCs allow low operating voltages, and their high birefringence allows reducing the thickness of the LC cells and hence reducing the switching time. The structures of the previously demonstrated LC lenses consist of hybrid lenses, use of either discrete electrodes, ${ }^{11}$ continuous highly resistive electrodes, ${ }^{12,13}$ or spatially distributed electric fields, ${ }^{14}$ binary diffractive lenses, ${ }^{15,16}$ polymer dispersed LC with gradient exposure, ${ }^{5}$ etc. Spectacle correction of presbyopic eyes using adaptive lenses is extremely important since a large population suffer from presbyopia. There are critical requirements on such a lens, including relatively large aperture, fast switching time, low voltages, high efficiency, high image quality, and power-off-safe design ${ }^{17}$ (i.e., when the electrical power is off, the LC lens is optically inactive; this mode is safe for distance-vision task such as driving), Recently we demonstrated a switchable diffractive LC lens ${ }^{17}$ that satisfies these requirements. In that design, the ring electrodes for modulating the eight-level (number of phase digitization) phase profile are patterned in one layer with a $1 \mu \mathrm{m}$ gap between the neighboring electrodes. In order to avoid the lateral gaps between the electrodes, we did a preliminary experiment with interleaved electrode pattern. ${ }^{18}$ However. with that design the number of phase levels is limited only to 4. Here we propose a novel and general design for a flat thin diffractive lens with high performance and powerful functions for vision care. It has a three-layer microfabricated electrode pattern and two-layer via structures for flexible interconnection and no-gap pattern. It allows any evennumbered phase levels greater than 4 and provides the capability of correction for near, intermediate, and distance

"Electronic mail: gli@optics.arizona.edu visions. Such a lens has the potential of revolutionizing the field of presbyopia correction.

The diffractive lens is designed at the peak of the human photopic response, $555 \mathrm{~nm}$. The radii of each zone and subzone are given by ${ }^{17}$

$$
r_{m, n}=\sqrt{2[(m-1)+n / L] \lambda f}, \quad n=1,2, \ldots, L,
$$

in which $m$ is a counting index that refers to successive zones starting in the center, $n$ the counting index of the individual phase levels, $L$ the number of phase levels per zone, $\lambda$ the wavelength, and $f$ the focal length. A nematic LC layer is sandwiched between a ground electrode substrate and a patterned electrode substrate. The ground electrode substrate contains a uniform conductive indium tin oxide (ITO) layer and the patterned electrodes are fabricated by photolithographic processing. For higher diffraction efficiency and ease of control, the patterned electrodes have a ring shape defined by Eq. (1). If all the ring electrodes for tailoring the refractive index are patterned in one layer, there must be gaps between neighboring electrodes. When the aperture of the lens is large, the gaps will distort the desired phase profile and hence reduce the diffraction efficiency. ${ }^{19}$ In order to avoid this effect, the odd-numbered and even-numbered electrodes are interleaved into two layers. The discrete electrodes for equal phase level in different zones are connected by a bus line through vias. The module was fabricated from the bottom to the top. All the bus lines are distributed in one layer in the bottom, and the number of the bus lines is equal to the number of phase levels in each zone. Therefore there are three layers of electrodes and two layers of vias in total. The layer for the bus lines, the layer for the odd-numbered electrodes, and the layer for the even-numbered electrodes are isolated by two insulator layers of $\mathrm{SiO}_{2}$, where vias are made for conduction between the bus lines and the ring electrodes. The fabrication procedure for the module is illustrated in sequences in Fig. 1. The etching time for $\mathrm{SiO}_{2}$ is optimized by checking the conductivity between ITO test structures, while the etching time for ITO is optimized by eliminating the electric shorts between different electrodes, 


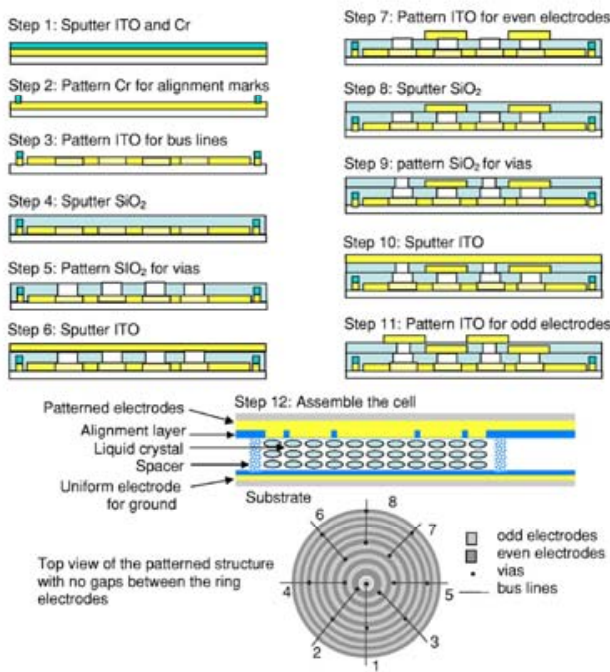

FIG. 1. (Color online) Processing steps for fabrication of the LC diffractive lens with three-layer patterned electrodes and two-layer via structures. At the bottom of the figure, the top view of the finished layers is shown with eight bus lines as an example. The design can be used for any higher phase levels.

i.e., electrodes for different phase levels. The new design makes it easier to fabricate lenses with higher-level phase steps and larger aperture and overcomes the shorts between the electrodes. This method can be used for design of LC lens of any phase levels. It should be noted that, unlike the conventional binary optics, in this design the increase of the phase levels (e.g., to 16 levels) in each zone does not increase the fabrication steps. The lens assembly procedure has been described in Ref. 17. The initial orientation of the molecules is parallel to the polarization of the incident beam, which is an extraordinary beam, and its effective refractive index can be changed in the range from $n_{e}$ to $n_{0}$ due to the reorientation of the $\mathrm{LC}$ molecule when a voltage is applied. The material has a positive dielectric anisotropy $(>0.2)$, which provides enough phase modulation for the visible wavelength with the 5 - $\mu \mathrm{m}$-thick cell.

A polarized optical microscope ${ }^{17}$ was used to inspect the lenses on a microscopic scale. Images of the eight-level 1 diopter lens under operation indicate that all eight electrode sets operated properly and provided discrete phase changes [Fig. 2(a)]. Eight drive voltages with amplitudes between 0 and $2 \mathrm{~V}_{\mathrm{rm}}$ were optimized and the maximum first-order diffraction efficiency (ratio of first-order diffracted power to the total transmitted power) of $92.5 \%$ was obtained for the whole lens area. The diffraction efficiency was measured as a function of the beam diameter. It reaches $94 \%$ near the center of the lens and decreases monotonically as the beam diameter is increased [Fig. 2(b)]. The diffraction efficiency at different locations of the lens is shown in Fig. 2(c). Although there is a small decrease due to the phase distortion caused by the fringing field at the zone boundaries, it is flatter than that of the design with gaps between the electrodes. The diffraction efficiency as a function of incidence angle is de-

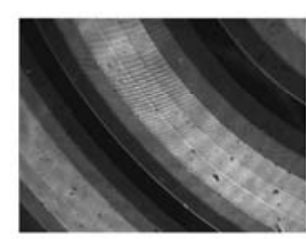

(a)
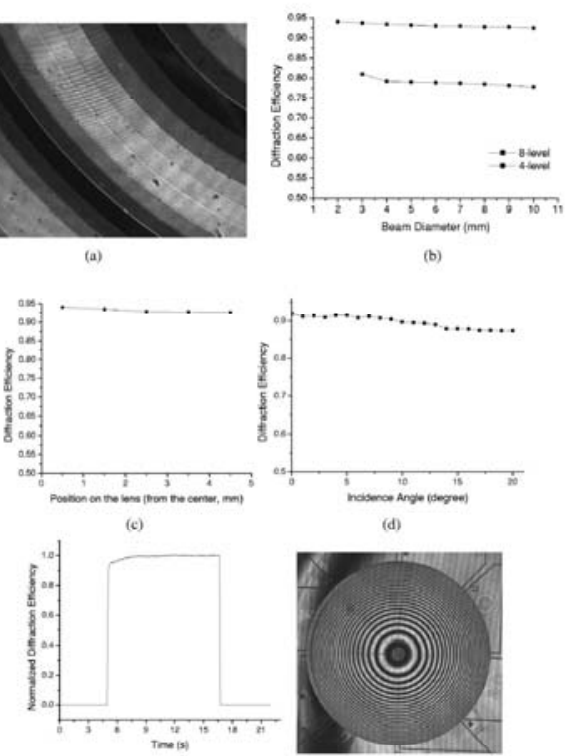

FIG. 2. Characterization of the LC lens. (a) Electro-optic response of individual electrode rings. [(b)-(d)] Diffraction efficiency as a function of the beam diameter, position on the lens, and incidence angle, respectively. (e) Switching dynamics of the lens. (f) Interferogram.

pieted in Fig. 2(d). It drops about $4 \%$ when the incidence angle is $20^{\circ}$. As shown in Fig. 2(e), switching of the lens is relatively fast. The rise time between $10 \%$ and $90 \%$ of the diffraction efficiencies was $150 \mathrm{~ms}$ and the full decay time was $134 \mathrm{~ms}$. The wave front immediately behind the lens can be measured using a phase-shift interferometer. Figure 2(f) shows one interferogram formed by the test wave and the reference wave. The rms error of the spherical wave front was measured to be 0.0688 waves. The parallelism between the two substrates of the lens may cause variation of the rms error. In addition, for the same lens, negative focusing power (-1 diopter) can also be achieved with the same diffraction efficiency by changing the sign of the slope of the applied voltages.

For vision correction of presbyopic eyes, polarization insensitive switchable lenses are needed. As homogeneously aligned nematic LC is polarization sensitive, two lenses with orthogonal buffing directions were integrated as a single polarization insensitive lens. Figure 3 depicts the alignment of the two lenses. The two lenses are mounted on translation stages. The incident light is linearly polarized at $45^{\circ}$. A charge coupled device (CCD) detector is put at the focal plane. When the two lenses are displaced, the two focal spots are separated (see upper right of Fig. 3). When the two lenses are well aligned, the two focal spots are overlapped (see lower right of Fig. 3) and the lenses are cemented together. For real application, the lenses will be centered on the line of sight when the user is looking at a near object, i.e., the locations of the centers of the LC lenses should be made to coincide with where the optical axes of the eyes pass through 


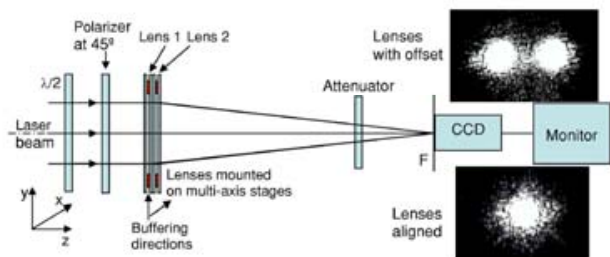

FIG. 3. (Color online) Assembly of a complete lens.

the spectacles when viewing a near object. This way any off-axis component is minimized and the optical performance is maximized. Optical simulation showed that the two lenses and the human eye model can generate good image spots. To demonstrate vision correction, a model eye was constructed using a fixed +60 diopter achromatic doublet glass lens and a CCD. The chromatic aberration of this system is $\sim 0.5$ diopter. The double-element LC lens was put in front of the model eye and an object was placed $\sim 35 \mathrm{~cm}$ in front of the lens with unpolarized white light illumination. When the complete LC lens is off, the model eye has insufficient power to form a sharp image [Fig. 4(a)]. But by switching on the diffractive lens, the image is brought into focus with excellent contrast [Fig. 4(b)].

Based on the current design, the focal length of the lens can be switched between a few digital values by reducing the zone period digitally, and each corresponds to a different diffraction efficiency. Let us assume an eight-level lens with the design focal length $f=F$ [Fig. 4(c)]. If each zone is divided into two, and voltage $V_{1}$ is applied to all the first and fifth subzones, $V_{2}$ to all the second and sixth subzones, $V_{3}$ to all the third and seventh subzones, and $V_{4}$ to all the fourth and eighth subzones, then the lens has a focal length of $F / 2$ with an efficiency corresponding to four-level phase digitization [lower part of Fig. 4(c)]. Actually every four subzones

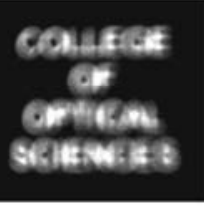

(a)

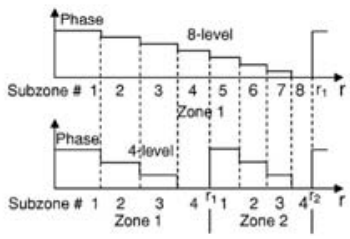

(c)

\section{COLLEGE} OF

OPTICAL

SCIENCES
COLLEGE OF OPTICAL SCIENCES

forms a new zone for the lens with the focal length $F / 2$. Furthermore, if each zone is divided into four, the lens has a focal length of $F / 4$ with an efficiency corresponding to a two-level device. In this case, every two subzones forms a new zone. With the eight-level 1 diopter lens described above, diffraction efficiency of $78 \%$ was obtained when it is optimized for the four-level 2 diopter case. The diffraction efficiency as a function of the lens aperture is shown in Fig. 2(b) and hybrid imaging with the model eye is shown in Fig. 4(d). For the same condition, the 2 diopter LC lens would allow the model eye to see objects at a closer distance than the 1 diopter lens. The main effect on the image is the brightness. Therefore the same electro-optic LC lens can be switched for near, intermediate, and distant visions with each using the whole lens aperture.

In conclusion, we have demonstrated a flat, thin, switchable LC diffractive lens with three-layer patterned electrodes and two-layer via structures, which is a general design for multilevel phase modulation. The lens has high optical quality and good response time, and this design allows larger aperture. The focusing powers of the whole aperture can be switched between different values. This kind of lens may afford an alternative to replace area division multifocal spectacle lenses. Antireflection coatings can be used to avoid light loss at the substrates. Usually, correction of presbyopia needs an add power less than 3 diopters and a $15 \mathrm{~mm}$ aperture for the center area is enough. In this case for an eightlevel lens, the smallest subzone is $\sim 3.1 \mu \mathrm{m}$. With the stateof-the-art facilities, it is feasible to make such lenses. For correcting a residual refractive error for myopia or hyperopia, a curved substrate can be used or the lens can be used together with a contact lens for eyes that need minor correction for distance vision. The other concern is the temperature dependence of the lens performance. The refractive indices of the LC change due to the temperature variation $\left(n_{e}\right.$ has a larger change than $n_{o}$ ). A temperature sensor and a variable voltage circuit are needed for compensation. The birefringence disappears above the clearing point in the isotropic phase.

'J. A. Davis, D. M. Cottrell, R. A. Lilly, and S. W. Connely, Opt. Lett. 14. 420 (1989).

'M. Ferstl and A.-M. Frisch, J. Mod. Opt. 43, 1451 (1996)

M. Hain, R. Glockner, S. Bhattacharya, D. Dias, S. Stankovic, and T. Tschudi, Opt. Commun. 188, 291 (2001).

C. W. Fowler and E. S. Pateras, Ophthalmic Physiol. Opt. 10. 186 (1990). SV. V. Presnyakov and T. V. Galstian, J. Appl. Phys. 97, 103101 (2005).

${ }^{6}$ H.-S. Ji, J.-H. Kim, and S. Kumar, Opt. Lett. 28, 1147 (2003).

${ }^{7}$ A. W. Lohmann, Appl. Opt. 9, 1669 (1970).

${ }^{8}$ H. J. Caulfield, Opt. Laser Technol. 34, 1 (2002)

'S. Sato, Jpn. J. Appl. Phys, 18, 1679 (1979).

S. Sato, Jpn. J. Appl. Phys, 18, 1679 (1979). (1985). A. Sugiyama, and R. Sato, Jpn. J. Appl. Phys., Part 2 24, L626 (1985).

"Y. Sun, G. P. Nordin, S. T. Kowel, and B. Wang, Proc, SPIE 4987, 209 (2003)

${ }_{12}^{12}$ N. A. Riza and M. C. DeJule, Opt. Lett. 19, 1013 (1994).

${ }^{13}$ A. F. Naumov, M. Y. Loktev, I. R. Guralnik, and G. Vdovin, Opt. Lett. 23, 992 (1998).

M. Ye and S. Sato, Mol. Cryst. Liq. Cryst. 422, 197 (2004).

$15 \mathrm{~J}$. S. Patel and K. Rastani, Opt, Lett. 16, 532 (1991)

${ }^{16}$ H. Ren, Y.-H. Fan, and S.-T. Wu, Appl. Phys. Lett. 83, 1515 (2003),

${ }^{17} \mathrm{G}$. Li, D. Mathine, P. Valley, P. Ayräs, J. Haddock, M. Giridhar, G. Williby, J. Schwiegerling, G. Meredith, B. Kippelen, S. Honkanen, and N. Peyghambarian, Proc. Natl. Acad. Sci. U.S.A. 103, 6100 (2006).

Peyghambarian, Proc. Natl. Acad. Sci. U.S.A. 103, $6100(2006)$.
${ }^{18}$ G. Li, P. Valley, M. Giridhar, D. Mathine, G. Meredith, J. Haddock, B.

Kippelen, and N. Peyghambarian, Appl. Phys. Lett. 89, 141120 (2006).

${ }^{19}$ B. Apter, U. Efron, and E. Bahat-Treidel, Appl. Opt. 43, 11 (2004).

FlG. 4. Hybrid imaging using the varifocal LC lens. The LC lens is (a) off and (b) activated with 1 diopter power. (c) An eight-level 1 diopter LC lens can be reconfigured to a four-level 2 diopter lens. (d) Hybrid imaging with the 2 diopter power.

to AIP license or copyright, see http://apl.aip.org/apl/copyright.jsp 
APPENDIX D

TUNABLE-FOCUS FLAT LIQUID-CRYSTAL DIFFRACTIVE LENS

\title{
Tunable-focus flat liquid-crystal diffractive lens
}

\author{
Pouria Valley,* David L. Mathine, Mohammad Reza Dodge, Jim Schwiegerling, \\ Gholam Peyman, and N. Peyghambarian \\ College of Optical Sciences, University of Arizona Tucson, Arizona 85721, USA \\ *Corresponding author: pouria $\oplus$ uarizona.edu \\ Received November 23, 2009; revised December 11, 2009; accepted December 14, 2009: \\ posted December 23, 2009 (Doc. ID 120292): published January 26, 2010 \\ We demonstrate an innovative variable-focus flat liquid-crystal diffractive lens (LCDL) with $95 \%$ diffraction \\ efficiency and millisecond switching times using a $\pm 2.4 \mathrm{~V}$ ac input. This lens is based on the electrical modu- \\ lation of a $3 \mu \mathrm{m}$ layer of nematic liquid-crystal sandwiched between a Fresnel zone electrode structure and \\ a reference substrate. Each zone is divided into 12 subzones to digitize the phase profiles and define the \\ subzones and re-estab \\ subzones and re-establishing the wrapping points. Potential applications include zooms with no movin \\ parts and autofocus lenses for compact imaging devices. (1) 2010 Optical Society of America \\ OCIS codes: $050.1965,230.3720,010.1080$.
}

Significant efforts have been made to create compact lenses with variable focal lengths and no moving parts for optical imaging devices with space constraints. Various methods for making such adaptivefocus lenses by shape change or refractive index change are currently under investigation including electrowetting, dielectric and membrane lenses, liquid-crystal refractive lenses [1], and surfacetension lenses [2]. Liquid-crystal diffractive lenses (LCDLs) were reported before, but they have low diffraction efficiency $[3,4]$. High-efficiency binary switchable LCDLs were reported by the authors for vision care applications [5-7], using a multielectrode driving scheme for generating diffractive optical elements [8].

In this Letter, we report on quasimonochromatic tunable-focus LCDL using flat binary Fresnel zone structured electrodes with multiple focal lengths and 12 phase levels fabricated on a thin layer of conductive, transparent indium-tin-oxide (ITO) using conventional microphotolithography techniques. By varying the refractive index of a very thin layer of a high-birefringence nematic liquid crystal (E7 from Merck), using the proper voltage distribution across the electrodes we can change the focal length of the lens.

Flat LCDLs have several advantages compared to the aforementioned techniques. First, very low power consumption with electronics drive voltages of less than $2.4 \mathrm{~V}$ (using a $\pm 2.5 \mathrm{~V}$ ac input) as compared to several tens of volts or above hundred volts required for most other lenses. Second, fast switching times of $<150 \mathrm{~ms}$ (focusing or rise) and $20 \mathrm{~ms}$ (defocusing or fall) compared to seconds for thick liquid crystal or liquid lenses. Third, scalability of the lens aperture up to $10 \mathrm{~mm}$ or more [5-7], whereas most other lenses are limited to about $5 \mathrm{~mm}$ or less for practical purposes. Fourth, small form factor with no need for a pump, actuator, servomotor, or considerable edge space that are needed for membrane and liquid lenses. Fifth, stability and robustness, insensitivity to vibrations, gravity, and capillary effects that limit membrane and electrowetting lenses. Sixth, high transmission compared to thick liquid crystal lenses with high scattering and absorption. Other benefits

include easy fabrication, safety against harmful leakage, and flatness. The disadvantages of our reported lens in its current form are polarization sensitivity and chromatic aberration, which are addressed in the experimental results section.

The lens module described in this Letter [Fig. 1(a)] is composed of a flat diffractive optical element (DOE) and a thin layer of liquid crystal sandwiched between two thin ITO glass substrates, one with the DOE pattern and one with no pattern used as the electronic ground. The effective refractive index of the liquid crystal can be varied with the applied voltage and together with the diffractive pattern that defines the phase-wrap points, phase profiles corresponding to various focal lengths can be achieved.

One type of DOE structure employed here is the binary Fresnel zone plate. Each zone is divided into 12
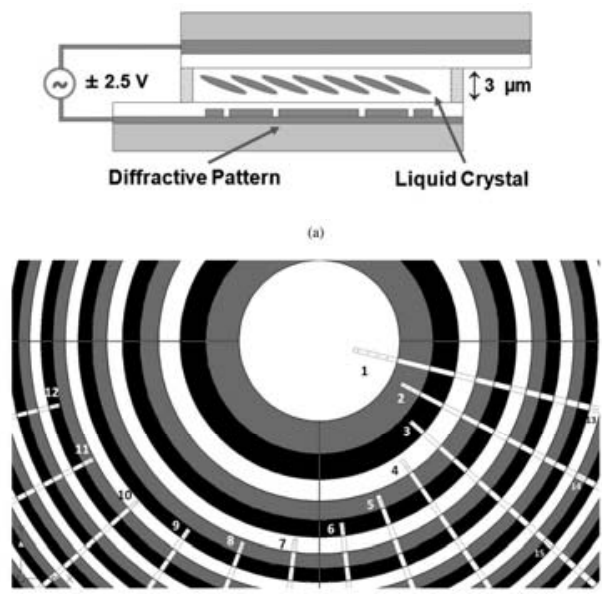

(b)

Fig. 1. Schematic of flat LCDL. (a) Side view. (b) Top view of the central zone and the shunting concept. In this example, every four electrodes are shunted to construct a three-level lens from a 12-level lens. Electrodes with the same color share the same voltages. 
subzones in a way that maintains the square-root-ofradius relationship so as to digitize the continuous quadratic phase transmission function [5-7]. Twelve electrical buses are established to shunt together all of the corresponding subzones in all of the zones. The subzone electrodes are separated by $1 \mu \mathrm{m}$ gaps to maintain electrical isolation between the electrodes. Isolation between bus bars and electrodes is achieved by a thin insulating layer of $\mathrm{SiO}_{2}$ that contains $2 \mu \mathrm{m}$ vias only at proper electrode locations where electrical contacts between the electrodes and corresponding bus bars are needed [Fig. 1(b)]. The narrowest electrode at the edge for the lens reported in this Letter has a width of $5 \mu \mathrm{m}$ with a radius of $3 \mathrm{~mm}$, and the innermost electrode has a radius of $192 \mu \mathrm{m}$.

Shunting of electrodes needs to be in a way that the correct retardation step sequence is achieved as moving outward radially. For example, going from 12 electrodes per zone to three electrodes per zone happens by electrically shunting the following subzones of all zones together so that the shunted electrodes will all have the same voltages: $(1,4,7,10),(2,5,8$, $11)$, and $(3,6,9,12)$ as shown in Fig. 1(b) by white, gray, and black regions. This way, the number of $2 \pi$ zone boundaries is increased by a factor of four, so a location that was labeled the zone boundary $m$ now becomes the zone boundary $4 \mathrm{~m}$. For example, the first zone boundary as marked by 12th subzone in Fig. 1(b) now becomes the fourth zone boundary. So the focal length is switched from $f$ to $1 / 4$ of $f$ based on the digitized Fresnel zone equation: $f=r_{m}^{2} / 2 m \lambda$, where $r_{m}$ is the radius of the $m$ th zone, $\lambda$ is the wavelength, and $m$ is an index denoting the $m$ th zone boundary [9]. Thus, by properly shunting every 2,3 , 4 , and 6 electrodes together we get $1 / 6,1 / 4,1 / 3$, and $1 / 2$ of $f$, respectively, in addition to the original design focal length $f$

Fabrication technique of the DOE for our lenses using conventional microphotolithography is similar to that of the cost effective semiconductor industry. The process involves deposition, patterning, and etching of the following thin films: $\mathrm{Cr}$ for alignment marks, ITO for bus lines and electrodes (150 nm thick with a resistivity of $20 \Omega / \square$ ), and $\mathrm{SiO}_{2}$ for vias.

The substrates are thoroughly cleaned with acetone, isopropyl alcohol, deionized water, ultrasonic cleaner, and oxygen plasma before each step. Thin films are deposited on a glass substrate using the Oxford IonFab $300+$ ion beam sputtering system. Then the substrate is spin coated with SPR-220 positive photoresist from Rohm-and-Hauss (R\&H) at $4000 \mathrm{rpm}$, soft baked at about $100^{\circ} \mathrm{C}$ on a hot plate, exposed to $350 \mathrm{~nm}$ UV light through a photomask using Karl-Suss MA6 aligner, developed using R\&H 352 developer for about $1 \mathrm{~min}$, and hard baked at about $120^{\circ} \mathrm{C}$ on a hot plate. The exposed, unpatterned areas on the films are etched away using the appropriate mixture of acids. Special care is needed to tightly control the process in terms of resolution and contamination, because the feature sizes are as small as $0.8 \mu \mathrm{m}$.

After fabrication of DOE is complete, nylon alignment layers are spin coated on the DOE substrate and the ITO reference electrode. Then, the layers are rubbed in antiparallel directions to provide homogeneous orientation for the liquid-crystal molecules. Glass fiber spacers are used to create a small and uniform gap of about $3 \mu \mathrm{m}$ between the two substrates to form a cell. The liquid crystal is drawn into the cell by the capillary action to fill the lens cell. Finally, wires are connected to ITO contact pads of the bus lines using silver paint to complete the assembly.

The lens is driven with twelve independently controllable voltage drivers. Each driver produces a modified square wave with a fundamental frequency of approximately $5 \mathrm{kHz}$ and voltages up to $\pm 2.5 \mathrm{~V}$. The waveforms are controllable with a dc analog input voltage that is adjusted to optimize the diffraction efficiency [10].

First, we measure the diffraction efficiency, which is defined as the fraction of light intensity passing through the lens that is focused into a specific diffraction order. The theoretical first-order diffraction efficiency of a binary $N$-level diffractive lens is given by: $\eta=\operatorname{sinc}^{2}(1 / N)$.

With twelve levels the maximum efficiency is $97.7 \%$ in theory. Figure 2 shows the values of the diffraction efficiency measured as a function of aperture size (circles) demonstrating more than $90 \%$ diffraction efficiencies. Figure 2 also depicts diffraction efficiency at various focal lengths (triangles) that correspond to different phase level digitization. The interelectrode gaps reduce the diffraction efficiency because of reduction in the effective area of the lens, and cause phase distortions owing to the fringing field effect near the zone boundaries [11]. These effects become weaker at smaller apertures because the gap size becomes small compared to the electrode widths moving toward the central zones and diffraction efficiency will increase. Fabrication errors such as over etching of the gaps can cause further reduction in the diffraction efficiency.

The response time of the lens was measured by recording the output of a high speed Si detector (Thorlabs DET10A) as a function of time. At the lowest optimal operating voltages (between 1.0 and $2.4 \mathrm{~V}$ ) the $10 \%-90 \%$ rise time of the intensity at the focal point

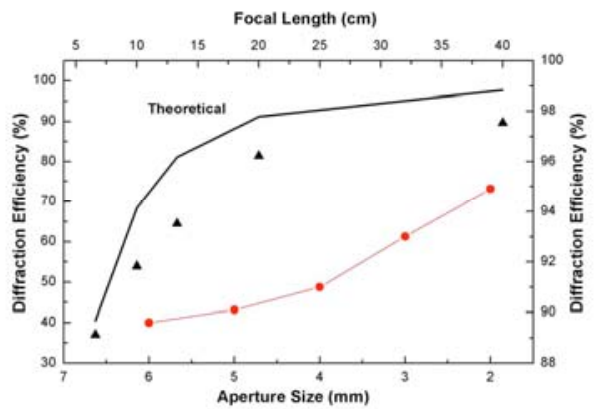

Fig. 2. (Color online) Diffraction efficiency measurements for various aperture sizes when $f=40 \mathrm{~cm}$ (circles) and for various focal lengths when the aperture diameter is $6 \mathrm{~mm}$ (triangles). 
was $150 \pm 6 \mathrm{~ms}$ (Fig. 3). The response time of each subzone is a function of the driving voltage across it [12]. Operating the lens at higher voltages results in faster response time as expected. For an average of $30 \%$ and $40 \%$ increase in the voltages, the response time was reduced to $105 \mathrm{~ms}$ and $55 \mathrm{~ms}$, respectively. The defocusing time for all sets of voltages was about $20 \mathrm{~ms}$.

Several imaging tests were performed with various target objects at different distances (Fig. 4). We used a broadband white light source to illuminate a photograph used as the object, with a $540 \mathrm{~nm}$ filter (10 nm FWHM to eliminate the chromatic aberration of lenses. The light reflected from object passes through a linear polarizer with its axis parallel to the buffing direction of the lens cell. A refractive lens $f$ $=50 \mathrm{~mm}$ ) is put in between the LCDL and the CCD camera to add more optical power to the system. The chromatic aberration of our lens can be reduced either by a proper hybrid design of a diffractive and refractive lens or by adoption of multiorder or harmonic diffraction using larger phase shift through employing thicker liquid crystals [13]. The need for a polarizer can also be eliminated by using two cells with orthogonal polarized buffing directions [5-7].

In this experiment the photograph was placed at different distances in front of the lens and was brought into focus employing different focusing powers of the LCDL (Fig. 4). The lens is originally designed as a 12 level, 2.5D $(f=40 \mathrm{~cm})$ lens that can be transformed to a six level, $5 \mathrm{D}(f=20 \mathrm{~cm})$; four level, $7.5 \mathrm{D}(f=13.3 \mathrm{~cm})$; three level, 10D $(f=10 \mathrm{~cm})$; and two level, 15D $(f=6.6 \mathrm{~cm})$ lens. The relative image sizes shown are exactly as captured by the CCD camera. Although the diffraction efficiency drops as going to shorter focal lengths, the image quality is still good. By making lenses with higher powers and greater number of phase levels, larger dynamic ranges and higher diffraction efficiencies can be achieved.

In conclusion, we have demonstrated a flat LCDL that is capable of providing multiple focal lengths with high optical qualities. It is compact, robust, operates at low voltage levels, and has fast switching dynamics, which makes it a good candidate for nonmechanical autofocus and zoom lenses in compact quasimonochromatic imaging systems.

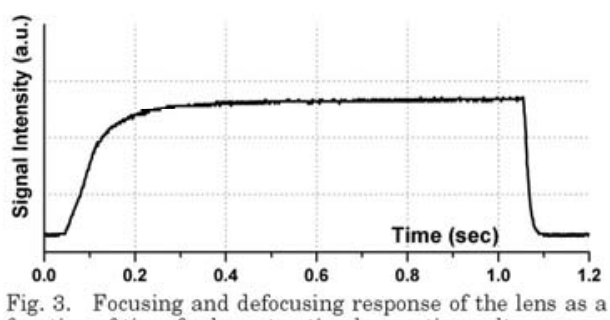

Fig. 3. Focusing and defocusing response of the lens as a function of time for lowest optimal operating voltages.

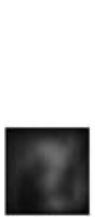

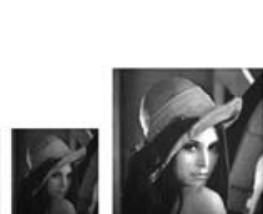

(c)

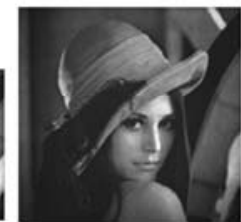

(d)

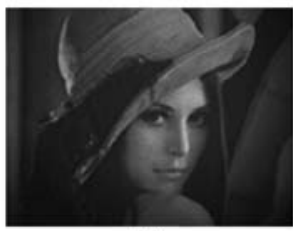

(e)

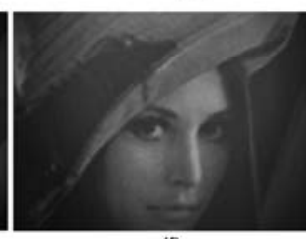

(f)
Fig. 4. Images taken from the object at different distances employing different focal lengths of the lens. (a) When LCDL is off (no voltages applied). (b) $f=40.0 \mathrm{~cm}$. (c) $f$ $=20.0 \mathrm{~cm}$. (d) $f=13.3 \mathrm{~cm}$. (e) $f=10.0 \mathrm{~cm}$. (f) $f=6.6 \mathrm{~cm}$.

The authors acknowledge support from the National Science Foundation (NSF) through CIAN ERC under grant EEC-0812072. We thank Veysi Demir and Michael Gaj for their collaborations.

\section{References}

1. H. Ren, S. Xu, Y. J. Lin, and S. T. Wu, Opt. Photonics News 19(10), 42 (2008).

2. C. A. López, C. Lee, and A. H. Hirsa, Appl. Phys. Lett. 87, 134102 (2005).

3. M. Hain, R. Glöckner, S. Bhattacharya, D. Dias, S. Stankovic, and T. Tschudi, Opt. Commun. 188, 291 (2001).

4. H. Ren, Y. H. Fan, and S. T. Wu, Appl. Phys. Lett. 83, 1515 (2003)

5. G. Li, D. L. Mathine, P. Valley, P. Äyräs, J. N. Haddock, M. S. Giridhar, G. Williby, J. Schwiegerling, G. R. Peyghambarian, Proc. Natl. Acad. Sci. USA 103, 6100 (2006).

6. G. Li, P. Valley, M. S. Giridhar, D. L. Mathine, G. Meredith, J. N. Haddock, B. Kippelen, and N. Peyghambarian, Appl. Phys. Lett. 89, 141120 (2006).

7. G. Li, P. Valley, P. Ayräs, D. L. Mathine, S. Honkanen, and N. Peyghambarian, Appl. Phys. Lett. 90, 111105 (2007).

8. P. F. McManamon, T. A. Dorschner, D. L Corkum, L. J, Friedman, D. S. Hobbs, M. Holz, S. Liberman, H. Q. Nguyen, D. P. Resler, R. C. Sharp, and E. A. Watson, Proc. IEEE 84, 268 (1996)

9. B. Kress and P. Meyrueis, Digital Diffractive Optics (Wiley, 2000).

10. T. Hoda, P. Ayras, L. LaRussa, N. Peyghambarian, and D. L. Mathine, Electron. Lett, 42, 1 (2006).

11. B. Apter, U. Efron, and E. Bahat-Treidel, Appl. Opt. 43, $11(2004)$

12. S. T. Wu, Appl. Opt. 28, 48 (1989)

13. D. W. Sweeney and G. E. Sommargren, Appl. Opt. 34, 2469 (1995) 
APPENDIX E

NONMECHANICAL BIFOCAL ZOOM TELESCOPE

\section{Nonmechanical bifocal zoom telescope}

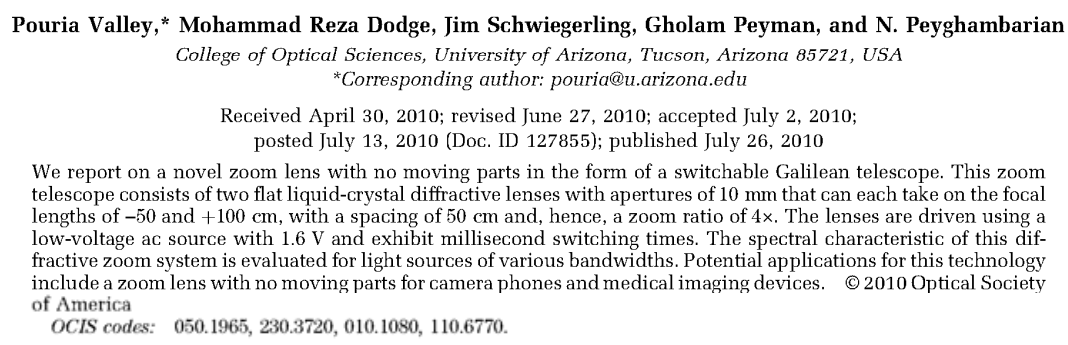

Zoom lenses with little [1-3] or no [4-11] mechanical movements have recently gained attention for imaging applications, such as mobile camera phones, in which size, cost, speed, complexity, and robustness have tight requirements. Other potential applications with simila requirements include medical imaging and vision correction devices. Traditional zoom lenses require several optical elements with delicate driving motors and precise mechanical cams to adjust the spacing between the groups of elements. This makes the system bulky, costly, relatively slow, complicated, and fragile.

To make a nonmechanical zoom lens, various methods have been investigated including electrowetting liquid lenses [4], pressure controlled fluidic lenses [5], reflective systems [6], deformable mirrors, and liquid-crystal spatial light modulators [7-11]. In each case, the foca length can actively be varied either by changing the surface shape (liquid lenses or deformable mirrors) or the refractive index (liquid-crystal lenses) by applying pressure or an electric field. Some of the aforementioned methods suffer from a need for a high voltage supply, as well as high power consumption, alignment issues, smal apertures, bulky or complicated peripheral drivers, and low dynamic range and resolution.

In this Letter, we demonstrate a low-voltage, nonmechanical zoom lens using two active liquid-crystal dif fractive lenses [12] in a Galilean telescope configuration. The Galilean telescope consists of a positive (convex) objective and a negative (concave) eye lens, separated by the summation of the focal lengths of the two lenses. Unlike the Keplerian or astronomical telescope, the Galilean telescope has a shorter overall length and produces an erect image with a magnification proportional to the ratio of the objective lens focal length to the eye lens focal length. A reversed Galilean telescope, similarly, is formed by using a negative objective and a positive eye lens. The lens spacing is the same as the Galilean telescope, but the systems have reciprocal magnification. This reversed system permits a large area of the visual field to be condensed into a small image. This is useful in vision correction for patients with visual field constriction, such as those with retinitis pigmentosa.

Both of the diffractive lenses used here have an aperture of $10 \mathrm{~mm}$, a maximum design focal length of $100 \mathrm{~cm}$ and eight levels of binary phase quantization, which results in 23 Fresnel zones each having eight subzones. The desired phase profile is achieved by electrically controlled effective birefringence of a nematic liquid crystal sandwiched between a flat Fresnel zone electrode substrate (Fig. 1) and a ground reference substrate, which both contain a transparent and conductive indiumtin-oxide (ITO) thin film

To maintain electrical isolation between the electrodes, either a small gap is introduced between them or the odd-numbered and even-numbered electrodes are formed in two separate layers with an insulating layer of $\mathrm{SiO}_{2}$ in between. The latter technique is adopted in this Letter, which gives an improved diffraction efficiency compared to the former technique because the interelectrode gaps are eliminated. However, the two-layer technique requires additional fabrication steps and accurate alignment of the odd and even electrodes. The electrodes of the same counting index for each subzone within each of the Fresnel zones are connected to bus bars through vias made in the insulating layer of $\mathrm{SiO}_{2}$.

Fabrication of these lenses involves the microphotolithography steps summarized in Fig. 2. After the photolithography process, both the patterned electrodes and the reference substrates are spin coated with a nylon alignment layer and are rubbed in the antiparallel direction to provide a homogeneous molecular orientation for the liquid crystal. Then, $2 \mu \mathrm{m}$ glass fiber spacers are dispersed onto the surface. E7 (Merck) liquid crystal is then used to fill the empty cell through capillary action at a

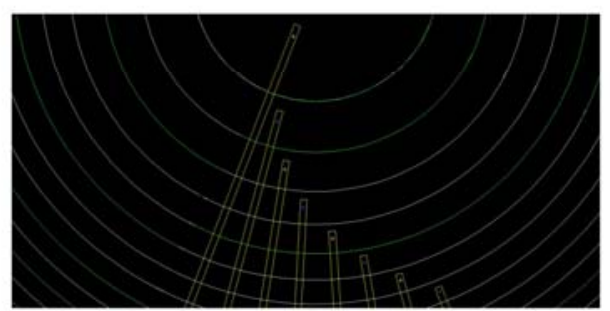

Fig. 1. (Color online) Top view of the Fresnel zone electrode structure near the center with $10 \mathrm{~mm}$ diameter and $100 \mathrm{~cm}$ focal length. Odd and even electrodes are patterned on two separate ITO layers, isolated by an $\mathrm{SiO}_{2}$ layer containing the vias that connect the bus lines and electrodes. 


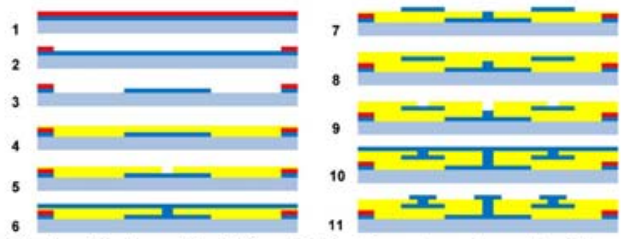

Fig. 2. (Color online) Microfabrication steps: 1, sputter $\mathrm{Cr}$ on an ITO glass substrate; 2 , pattem the alignment marks; 3 , pattern the odd electrodes; 4 , sputter $\mathrm{SiO}_{2} ; 5$, pattern the first vias; tern the odd electrodes; 4 , sputter $\mathrm{SiO}_{2} ; 5$, pattern the first vias,
6 , sputter ITO; 7 , pattem the even electrodes; 8 , sputter $\mathrm{SiO}_{2} ; 9$, pattem the second vias; 10 , sputter ITO; 11 , pattem the bus lines (all films are $150 \mathrm{~nm}$ thick.

temperature above the clearing point $\left(60^{\circ} \mathrm{C}\right)$. The cell is then cooled at $1{ }^{\circ} \mathrm{C} / \mathrm{min}$ to room temperature. Finally, the cell is sealed and connected to drive electronics through a set of thin stranded wires.

A simple resistive circuit with $L$ potentiometers, where $L$ is the number of the binary phase level quantization, is used to drive the diffractive lenses (Fig. 3). The resistances and the input voltage, and, hence, the driving electric field across different Fresnel subzones, is adjusted to introduce the appropriate phase shift for maximum diffraction efficiency. The voltages are applied simultaneously and are monotonically increasing from the first to the last subzone. The focal lengths can electronically be switched to fractions of the maximum foca length in milliseconds [13]. The sign of the focal length can easily be changed by reversing the order in which the voltages are applied to the lenses and, hence, reversing the slope of the phase profile. Therefore, one can produce a focal length of $50 \mathrm{~cm}$ from a $100 \mathrm{~cm}$ lens by properly shunting every two electrodes and a simple rearrangement of the voltage distribution.

For the imaging experiments, we used a fixed refractive lens with an object placed at its front focal point so as to collimate the rays entering the diffractive telescope, as shown in Fig. 4. Another fixed refractive lens was placed in the image space behind the second diffractive lens to focus the collimated rays emerging from the telescope onto a CCD camera positioned at the back foca distance of the refractive lens. We also used a linear polarizer with its axis parallel to the buffing direction (i.e. the alignment direction of the liquid-crystal molecules) in the diffractive lenses. Since the polarizer is placed behind the diffractive lens, it has no effect on the diffraction efficiency. The polarization dependence of each lens can be eliminated by addition of a second diffractive lens with orthogonal buffing direction. A 1951 USAF resolution test chart was used as the object.

When both of the diffractive lenses are off (no voltages applied), they have no optical power, and the object is imaged onto the CCD with a magnification dictated solely by the refractive lenses [Fig. 5(b)]. When a voltage is applied to the diffractive lenses such that a reversed Galilean configuration with $f_{\text {obj }}=-50 \mathrm{~cm}, f_{\text {eye }}=100 \mathrm{~cm}$

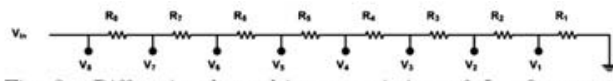

Fig. 3. Diffractive lens driver consisting of $L=8$ potentiometers. $V_{\text {in }}$ is a square wave of $1.6 \mathrm{~V}$ and $1 \mathrm{KHz}$.
August 1, 2010 / Vol. 35, No. 15 / OPTICS LETTERS

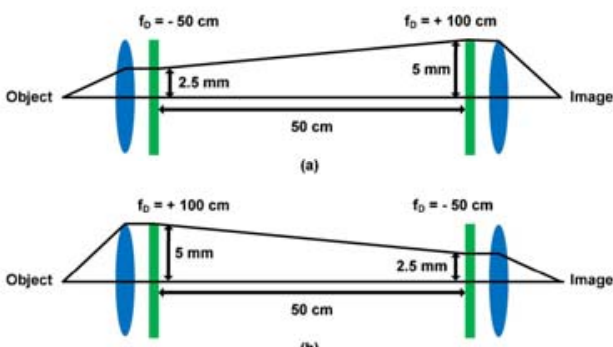

(b)

Fig. 4. (Color online) Schematic of the nonmechanical bifocal zoom lens in (a) reversed Galilean (zoom out) and (b) Galilean (zoom in) configurations. Fixed-focus refractive lenses (blue ellipse) have the same focal length, but the focal length of the diffractive lenses (green rectangles) is switched electronically. Graph not to scale.

[Fig, 4(a)] is obtained, the image is minified by a factor of $1 / 2$ [Fig. 5(a)]. When the voltage is adjusted to the Galilean configuration with $f_{\mathrm{obj}}=100 \mathrm{~cm}, f_{\text {eye }}=-50 \mathrm{~cm}$ [Fig. 4(b)], the image is magnified by a factor of 2 [Fig. 5(c)]. Here, a quasi-monochromatic source with a $10 \mathrm{~nm}$ bandwidth is used. Also, both of the refractive lenses have the same focal length of $5 \mathrm{~cm}$, but, if the focal lengths are chosen differently, the system magnification in each state would be the product of the telescope magnification and the ratio of the refractive lenses focal lengths. However, the overall magnification or zoom ratio is given by $\left(f_{\text {obj }} / f_{\text {eye }}\right)^{2}$, which is $4 \times$ for our case.

The measured diffraction efficiency of the eight-level diffractive lenses used here is about $85 \%$ (as compared with $95 \%$ in theory), and, when shunted to produce the focal length of $50 \mathrm{~cm}$ from the original $100 \mathrm{~cm}$ design focal length, the efficiency drops to about $65 \%$ because the quantization level of the diffractive lens is reduced to a four-level system ( $81 \%$ theoretical) [13]. So the overall efficiency for this zoom system is $0.65 \times 0.85=0.55$ for both configurations. By using diffractive lenses with a larger number of phase quantization, the diffraction efficiency will increase and the image quality and throughput will improve. Also, by designing diffractive lenses with shorter focal lengths, the overall length of the zoom system can be reduced and the zoom ratio is increased. We intend to report on these improvements in our future work, as well as explore zoom lenses with arbitrary configuration and intermediate zooming steps.

The fringing fields effect on the phase profile has a significant role in reducing the efficiency. This effect is even
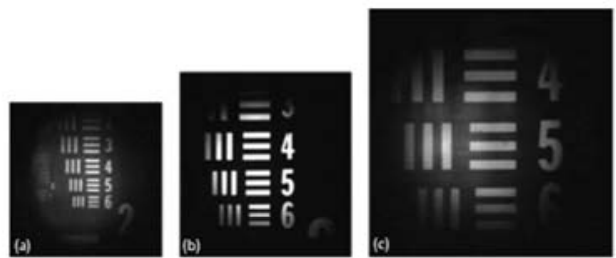

Fig. 5. Images taken with the zoom lens when (a) zoomed out, (b) no zoom, and (c) zoomed in, demonstrating $4 \times$ overall magnification. 

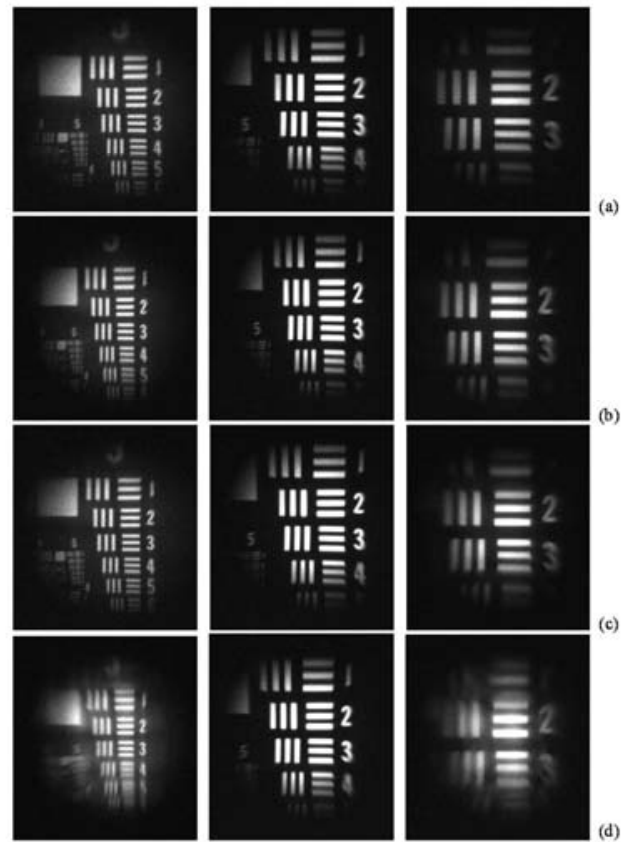

Fig. 6. Images taken with a white light source passing through different bandpass filters with bandwidths of (a) 10 , (b) 30 , (c) 90 , and (d) $300 \mathrm{~nm}$

more important when the electrodes are rearranged to increase the optical power. This is the main reason that the image quality is slightly degraded when zooming in or out. The liquid-crystal dispersion and the spectral bandwidth contribute to a lower efficiency, too. The efficiency falloff, which is up to $10 \%$ here, can be lowered or minimized when a material with a lower dispersion is used or when the birefringence is an increasing function of the wavelength.

To examine the spectral characteristics of our diffractive zoom lens, four imaging experiments were performed using light sources of various bandwidths. Four different bandpass filters with bandwidths of $10,30,90$, and $300 \mathrm{~nm}$ were placed in front of a broadband whitelight source and three images (zoomed out, no zoom, and zoomed in) were taken for each case. All other parameters of the setup remained the same. Because of the strong wavelength dependence of the diffractive lenses, some chromatic aberration is expected under a broadband light source. As seen in Fig. 6, for a broadband light of $300 \mathrm{~nm}$, the image quality is degraded consider- ably [Fig. 6(d)], but up to $90 \mathrm{~nm}$ the image quality is still adequate [Figs. 6(a)-6(c)]. Therefore, by using a sequential image acquisition algorithm, a full-color image can be obtained from a sequence of quasi-monochrome redgreen-blue (RGB) images and by combining those RGB images by electronic post-image-processing using software correction. Alternatively, the chromatic aberration of diffractive lenses can be reduced either by a proper hybrid design of a diffractive and refractive lens or by adoption of multiorder or harmonic diffraction using a larger phase shift through employing thicker liquid crystals [13].

In conclusion, we have demonstrated a novel zoom lens in a telescopic configuration using two active liquidcrystal diffractive lenses. This configuration can quickly change the focus and zoom with no macroscopic moving parts. It can produce images of adequate quality for light bands of up to $100 \mathrm{~nm}$. A few different methods can be used to obtain full-color images with small aberrations. Potential applications include compact and lightweight cameras in cell phones, webcams, and medical and ophthalmic imaging devices.

The authors acknowledge National Science Foundation (NSF) support via the Center for Integrated Access Network Engineering Research Center, grant EEC0812072, and thank David Mathine, Michael Gaj, and Pekka Äyräs for their collaboration.

\section{References}

1. J. Schwiegerling and C. Paleta-Toxqui, Appl. Opt. 48, 1932 (2009).

2. S. C. Park, S. H. Lee, and J. G. Kim, J. Opt. Soc. Korea 13, 206 (2009).

3. S. Kuiper, B. H. W. Hendriks, J. F. Suijver, S. Deladi, and I. Helwegen, Proc. SPIE 6466, 64660F (2007).

4. R. Peng, J. Chen, and S. Zhuang, J. Opt. Soc. Am. A 25, 2644 (2008).

5. D. Y. Zhang, N. Justis, and Y. H. Lo, Opt. Commun. 249 , 175 (2005).

6. K. Seidl, J. Knobbe, and H. Grüger, Appl. Opt. 48, 4097 (2009).

7. D. V. Wick, "Active optical zoom system," U.S. patent 6,977,777 (June 18, 2004).

8. D. V. Wick, T. Martinez, D. M. Payne, W. C. Sweatt, and S. R. Restaino, Proc. SPIE 5798, 151 (2005).

9. B. E. Bagwell, D. V. Wick, R. Batchko, J. D. Mansell, T. Martinez, S. Serati, G. Sharp, and J. Schwiegerling, Proc. SPIE 6289, 628908 (2006)

10. T. Martinez, D. V. Wick, D. M. Payne, J. T. Baker, and S. R. Restaino, Proc. SPIE 5234, 375 (2004).

11. E. C. Tam, Opt. Lett. 17, 369 (1992).

12. P. Valley, D. L. Mathine, M. R. Dodge, J. Schwiegerling, G. Peyman, and N. Peyghambarian, Opt. Lett. 35, 336 (2010).

13. D. W. Sweeney and G. E. Sommargren, Appl. Opt. 34, 2469 (1995). 


\title{
ADJUSTABLE HYBRID DIFFRACTIVE/REFRACTIVE ACHROMATIC LENS
}

\author{
Pouria Valley ${ }^{*}$, Nickolaos Savidis, Jim Schwiegerling, Mohammad Reza Dodge, Gholam Peyman, \\ and N. Peyghambarian \\ College of Optical Sciences, University of Arizona Tucson, AZ 85721 USA \\ "pouria@u.arizona.edu
}

\begin{abstract}
We demonstrate a variable focal length achromatic lens that consists of a flat liquid crystal diffractive lens and a pressure-controlled fluidic refractive lens. The diffractive lens is composed of a flat binary Fresnel zone structure and a thin liquid crystal layer, producing high efficiency and millisecond switching times while applying a low ac voltage input. The focusing power of the diffractive lens is adjusted by electrically modifying the sub-zones and re-establishing phase wrapping points. The refractive lens includes a fluid chamber with a flat glass surface and an opposing elastic polydimethylsiloxane (PDMS) membrane surface. Inserting fluid volume through a pump system into the clear aperture region alters the membrane curvature and adjusts the refractive lens' focal position. Primary chromatic aberration is corrected through the coupling of the fluidic and diffractive lenses at selected focal lengths. Potential applications include miniature color imaging systems, medical and ophthalmic devices, or any design that utilizes variable focal length achromats.
\end{abstract}

(C)2010 Optical Society of America

OCIS codes: (050.1965) Diffractive lens; (230.3720) Liquid-crystal devices; (80.3630) Lenses.

\section{References and links}

1. Donald C. O'Shea, Thomas J. Suleski, Alan D. Kathman, Dennis W. Prather, Diffractive Optics: Design, Fabrication, and Test (SPIE Press Book, 2004).

2. P. Valley, D. L. Mathine, M. R. Dodge, J. Schwiegerling, G. Peyman, and N. Peyghambarian, "Tunable-focus flat liquidcrystal diffractive lens," Opt. Lett. 35, 336-338 (2010).

3. P. Valley, M. R. Dodge, J. Schwiegerling, G. Peyman, and N. Peyghambarian, "Nonmechanical bifocal zoom telescope," Opt. Lett. 35, 2582-2584 (2010).

4. S. Sato, A. Sugiyama and R. Sato, "Variable-Focus Liquid-Crystal Fresnel Lens,” Jpn. J. Appl. Phys. 24 pp. L626-628 (1985)

5. H. Ren, Y.H. Fan, and S.T. Wu, "Tunable Fresnel lens using nanoscale polymer-dispersed liquid crystals," Appl. Phys. Lett. 83, 1515-1517 (2003).

6. B.E. Bagwell, D.V. Wick, R. Batchko, J.D. Mansell, T. Martiez, S. Serati, G. Sharp, and J. Schwiegerling, "Liquid crystal based active optics," Proc. SPIE 6289, 628908 (2006).

7. D.Y. Zhang, N. Justis, and Y.H. Lo, "Integrated fluidic adaptive zoom lens," Opt. Lett. 29, 2855-2857 (2004).

8. H. Ren, D. Fox, P. A. Anderson, B. Wu, and S.T. Wu, "Tunable-focus liquid lens controlled using a servo motor," Opt. Express 14, 8031-8036 (2006)

9. R. Marks, D. L. Mathine, G. Peyman, J. Schwiegerling, and N. Peyghambarian, "Adjustable fluidic lenses for ophthalmic corrections," Opt. Lett. 34, 515-517 (2009).

10. T. Stone, N. George, "Hybrid diffractive-refractive lenses and achromats," Appl. Opt. 27, 2960-2971 (1988)

11. G. Zhou, H. M. Leung, H. Yu, A. S. Kumar, and F. S. Chau, "Liquid tunable diffractive/refractive hybrid lens," Opt. Lett. 34, 2793-2795 (2009).

12. John E. Greivenkamp "Field guide to geometrical optics," SPIE Press (2004)

13. M. Daimon and A. Masumura, "Measurement of the refractive index of distilled water from the near-infrared region to the ultraviolet region," Appl. Opt. 46, 3811-3820 (2007) 


\section{Introduction}

A traditional achromatic lens or achromat corrects for primary chromatic aberration by matching the focal points of a red and a blue wavelength. The dispersion is reduced over the visible spectrum, however residual secondary chromatic aberration remains at the green wavelength. Achromatic doublets consist of a crown glass followed by a flint. The crown glass has a positive focal length with low dispersion while the flint has a negative power with high dispersion. The combination of these two glass disparities is designed to match the red and blue wavelengths to a common focus along the optical axis (z-axis) to eliminate primary chromatic aberration.

It is known that diffractive lenses have strong chromatic aberration [1]. We have previously reported on designing and demonstrating adjustable focus diffractive lenses using liquid crystal as the variable index medium $[2,3]$. In this paper, we present an adjustable liquid crystal diffractive lens and an adjustable pressure-controlled fluidic lens that in combination (Figure 1) that minimizes primary chromatic aberration. Individually, the tunable liquid crystal diffractive lenses [2-6] and fluidic lenses [7-9] have a variable focal length. At each focal location chromatic aberration is observable when uncorrected. Chromatic aberration is greater in elements with larger dispersion, such as diffractive lenses, and becomes more significant for materials with lower Abbe numbers. Diffractive and holographic lenses have been proposed to replace the traditional achromatic doublets for fixed focal length designs [1, 10]. More recently, a useful hybrid variable focal length fluidic/diffractive lens was demonstrated; however, the diffractive component applies a fixed focal length [11]. We have extended this work to include a variable focal length diffractive component. In effect, we have created a variable achromatic doublet with no moving parts.

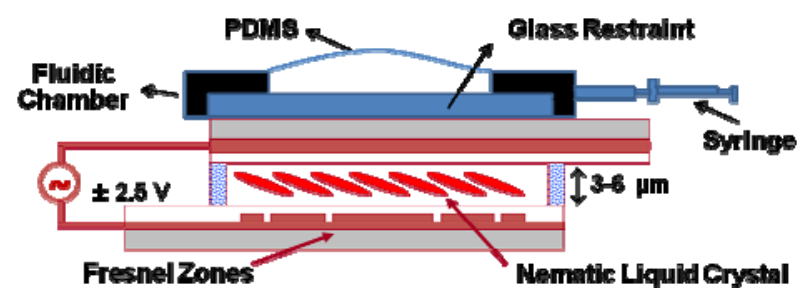

Fig. 1. Schematic of the hybrid liquid crystal diffractive lens and fluidic lens.

Diffractive lenses have an Abbe number much smaller than their refractive counterparts [1]. The Abbe number of a diffractive lens is unique in that it is equal to -3.45 , solely dependent upon the specified Fraunhofer $d, F, C$ wavelengths, as given by $\mathrm{V}_{\text {diffractive }}=\lambda_{d} /\left(\lambda_{F}-\lambda_{C}\right)[1,10]$. The focal length of a Fresnel zone-based diffractive lens is given by $f=r_{l}{ }^{2} /(2 \lambda)$ where $r_{l}$ is the radius of the first Fresnel zone and $\lambda$ is the wavelength of the incident light [1]. Therefore, the focal length at any other wavelength $\lambda$, in nm, can be scaled by the design wavelength according to $f(\lambda)=(555 / \lambda) f_{d}$ where $f_{d}$ is the design focal length at 555 nm.

An approach must be established to correlate the diffractive lens with the refractive lens. For certain focal lengths of the diffractive lens, $f_{d}$, we may choose the focal lengths and Abbe number of the fluidic lens such that they satisfy the achromat equation: $f_{f} V_{f}-3.45 f_{d}=0$, where $f_{f}$ and $V_{f}$ are the focal length and Abbe number of the fluidic lens, respectively. Refractive lenses have an Abbe number that is related to the indices of refraction as specified by the Fraunhofer $d, C, F$ lines where $\mathrm{V}_{\text {fluidic }}=\left(n_{d}-1\right) /\left(n_{F}\right.$ $\left.-n_{C}\right)$. By knowing the focal length of the diffractive lens and the Abbe numbers of the refractive and diffractive lenses, one is able to determine the focal length range of the fluidic lens required to achieve an achromat. Table 1 identifies which focal length ranges are necessary for a variable fluidic lens to produce an achromat with diffractive lenses ranging between $67 \mathrm{~mm}$ and $1000 \mathrm{~mm}$ focal lengths at specified Abbe numbers of the refractive lens. Optical glasses have an Abbe range between 25 and 65 [12], however using optical fluids increases this range of achievable Abbe numbers. Once the focal lengths are determined to identify what is necessary to match the diffractive lenses, the proper Abbe number and hence fluid may be chosen to achieve this goal. 


\section{Liquid Crystal Diffractive Lens}

We began by designing diffractive elements with known focal lengths and Abbe numbers. We developed two variable focal length diffractive lenses. Diffractive lens A possesses a design focal length of $1000 \mathrm{~mm}$ at $\lambda=555 \mathrm{~nm}$ with a clear aperture of $10 \mathrm{~mm}$ and eight binary phase quantization that results in a maximum diffraction efficiency of $94.9 \%$ in theory. Diffractive lens B possesses a design focal length of $400 \mathrm{~mm}$ at $\lambda=555 \mathrm{~nm}$ with a clear aperture of $6 \mathrm{~mm}$ and twelve levels of binary phase quantization that results in a maximum diffraction efficiency of $97.7 \%$ in theory. By properly shunting the electrodes, the diffractive lens A can provide focal lengths of $1000 \mathrm{~mm}, 500 \mathrm{~mm}$, and $250 \mathrm{~mm}$, and the diffractive lens B can provide $400 \mathrm{~mm}, 200 \mathrm{~mm}, 133 \mathrm{~mm}, 100 \mathrm{~mm}$, and $67 \mathrm{~mm}$ focal lengths. [2, 3]

Desired phase profiles are achieved by shifting the effective refractive index of a nematic liquid crystal. The nematic liquid crystal is sandwiched between a flat Fresnel zone electrode substrate and a ground reference substrate, where both substrates contain a transparent and conductive Indium-Tin-Oxide (ITO) layer. To maintain the electrical isolation between the electrodes in the diffractive lens A, the oddnumbered and even-numbered electrodes are formed in two separate layers with an insulating layer of $\mathrm{SiO}_{2}$ in between [3]. For the diffractive lens B, one-micron gaps are implemented as isolators between the electrodes that reduce the fabrication steps [2]. The electrodes (or subzones) of the same counting index within each of the Fresnel zones are connected to bus bars through vias made in the insulating layer of $\mathrm{SiO}_{2}$.

Fabrication of our diffractive lenses involves a few steps of deposition, lithography and etching. Ion beam sputtering was the deposition method used to produce uniform films of around $150 \mathrm{~nm}$ thick. Then photo-lithography was carried out using a diluted S-1805 photoresist (from Rohm and Hoss) and Karl-Suss MA6 mask aligner. The patterns were then etched using the appropriate acids/etchants for each layer. After the micro-fabrication process, both the patterned electrodes and the reference substrates are spin-coated with a nylon alignment layer. The substrates are then baked at $115^{\circ} \mathrm{C}$, buffed unidirectionally, and put together in the anti-parallel geometry to provide a homogeneous molecular orientation for the liquid crystal. Glass fiber spacers are used in the cell assembly. The lens cell is filled with the liquid crystal (E7 from Merck) via the capillary action at a temperature above the clearing point $\left(60^{\circ} \mathrm{C}\right)$ and cooled slowly to the room temperature. Finally, the cell is sealed and connected to drive electronics through a set of thin stranded wires.

Two resistive circuits with eight and twelve potentiometers drive the diffractive lenses. The resistances and the input voltage, hence the driving electric field across different Fresnel subzones, are adjusted to introduce the appropriate phase shift for the maximum diffraction efficiency. The voltages are applied simultaneously and are monotonically increasing from the first to the last subzone. The focal lengths are electronically switchable to fractions of the maximum design focal length in milliseconds. It is also possible to achieve negative focal lengths by reversing the order in which the voltages are applied to the diffractive lenses, thus reversing the slope of the phase profile. $[2,3]$ 


\section{Fluidic Refractive Lens}

The refractive lens is a plano-convex singlet with a predetermined amount of fluid inserted into the lens chamber. During preparation, fluid is inserted in excess within the chamber as to induce a vacuum pressure to evacuate air. If the air is not evacuated then it is treated as a second index within the clear aperture, resulting in a drastic alteration of desired lens properties. Only the membrane curvature changes when fluid is pumped into the chamber since the frame is metal and the opposing side is transparent glass.

The deformable membrane layer is a moldable optically clear elastomer with uniform thickness. Fabrication of the deformable membrane involves a pre-backing process while the PDMS mixture is formulated within molds designed on a flat $5 \lambda$ glass surface. The mixture is deposited within the molds which are then stirred within a vacuum system as to remove the excess air within the PDMS. The thickness of the membrane depends on the amount of material deposited within each mold. After the air is evacuated, the PDMS is placed into an oven at $90^{\circ} \mathrm{C}$ for an hour. We then remove the PDMS and peel off the membrane layers, which have a designed thickness of approximately 30-120 microns [9]. The membrane layers are removed with nylon tweezers and trimmed to remove excess material. Removal of excess material is necessary as to ensure a flat membrane to be clamped into the chamber.

The chamber is a metal frame which the membrane locks into, possessing a clear aperture within the center and flanges on its periphery. Control of the lens' output shape is achieved by controlling the clear aperture's opto-mechanical shape, where this clear aperture has a circular shape as to produce a rotationally symmetric fluidic lens. There is a retainer ring that has equal and opposite flanges relative to the metal frame. Through pressure, the membrane is applied onto the flat metal frame and the retainer ring locks the membrane onto the frame. This flat frame is then aligned and squeezed into a two part assembly, creating the singlet chamber. The chamber is then mounted onto a frame that has openings to place onto a rail. The chamber posses a single fluidic fitting that connects the fluid chamber to a syringe.

A syringe is placed into a pump system that alters the fluid output, permitting for control of the fluidic lens' radius of curvature and focal length. The applied pump controllers operate at a maximum of $0.0125 \mathrm{ml} / \mathrm{sec}$, an operation rate of $50 \mu \mathrm{l}$ in 4 seconds. This corresponds to a focal shift of about $10 \mathrm{~mm}$ when there is high lens curvature and a shift of about 50 to $100 \mathrm{~mm}$ per $50 \mu \mathrm{l}$ evacuation at lower curvatures. Due to drastic fluid removal effects at flatter curvatures, it was opted to decrease the amount of fluid inserted or removed at higher radii of curvature. A plano-convex lens is developed over this region and only positive focal lengths are selectively outputted.

\section{Methods and Test Results}

It is non-trivial to place the proper fluid into the chamber when compensating for the diffractive lens. Identifying the proper fluid came from a four step process. Firstly, one must identify the membranes radius of curvature range. This allows for one to physically characterize the limitations of the fluidic lens. Also, by identifying the radius of curvature with a known fluid, it is possible to quantify the focal length range of any fluid by knowing the new fluid's index of refraction. Once one knows the achievable radii of curvature and focal lengths of the fluidic lens, it is necessary to specify the focal lengths needed to compensate for the diffractive lens. For our experimental setup, we have already specified the Abbe number of the diffractive lenses and also the focal lengths achievable by our diffractive lenses. Table 1 took these values into consideration and found the focal length solutions of fluids at a wide scope of Abbe values. Therefore, we match the physical focal length range of the fluidic lens to a reasonable Abbe number so that a high percentage of achromatic doublets are achievable. The final step is to identify a fluid with the proper index of refraction and Abbe number as was previously assessed. It is also important that the fluid found is non-reactive or absorptive with the membrane that one is applying. Through this approach we satisfy the achromat equation: $f_{1} V_{1}+f_{2} V_{2}=0$, where $V$ is the Abbe number. 
Table 1. Values needed for fluidic lens focal lengths fg (in $\mathbf{~ m m}$ ) at different fluid Abbe number values and given diffractive lens focal lengths at the design wavelength of $555 \mathrm{~nm}$.

\begin{tabular}{|c|c|c|c|c|c|}
\hline $\begin{array}{l}\text { Diffractive lens powers } \\
\text { and focal lengths }\end{array}$ & $\begin{array}{l}\text { Needed fg if } \\
\text { Vf }=25\end{array}$ & $\begin{array}{l}\text { Needed fg if } \\
\text { Vf }=30\end{array}$ & $\begin{array}{l}\text { Needed fg if } \\
\text { Vf }=35\end{array}$ & $\begin{array}{l}\text { Needed fg if } \\
\text { Vf }=40\end{array}$ & $\begin{array}{c}\text { Needed fg if } \\
\text { Vf }=45\end{array}$ \\
\hline $1 \mathrm{D}(1000 \mathrm{~mm})$ & 690.00 & 345.00 & 252.56 & 230.00 & 172.50 \\
\hline $2.0 \mathrm{D}(500 \mathrm{~mm})$ & 345.00 & 172.50 & 126.28 & 115.00 & 86.25 \\
\hline $2.5 \mathrm{D}(400 \mathrm{~mm})$ & 276.00 & 138.00 & 101.02 & 92.00 & 69.00 \\
\hline $4.0 \mathrm{D}(250 \mathrm{~mm})$ & 172.50 & 86.25 & 63.14 & 57.50 & 43.13 \\
\hline $5.0 \mathrm{D}(200 \mathrm{~mm})$ & 138.00 & 69.00 & 50.51 & 46.00 & 34.50 \\
\hline $7.5 \mathrm{D}(133.33 \mathrm{~mm})$ & 92.00 & 46.00 & 33.67 & 30.67 & 23.00 \\
\hline $10 \mathrm{D}(100 \mathrm{~mm})$ & 69.00 & 34.50 & 25.26 & 23.00 & 17.25 \\
\hline $15 \mathrm{D}(66.66 \mathrm{~mm})$ & 46.00 & 23.00 & 16.84 & 15.33 & 11.50 \\
\hline $20 \mathrm{D}(50 \mathrm{~mm})$ & 34.50 & 17.25 & 12.63 & 11.50 & 8.63 \\
\hline $25 \mathrm{D}(40 \mathrm{~mm})$ & 27.60 & 13.80 & 10.10 & 9.20 & 6.90 \\
\hline $30 \mathrm{D}(33.333 \mathrm{~mm})$ & 23.00 & 11.50 & 8.42 & 7.67 & 5.75 \\
\hline $35 \mathrm{D}(28.57 \mathrm{~mm})$ & 19.71 & 9.86 & 7.22 & 6.57 & 4.93 \\
\hline $40 \mathrm{D}(25 \mathrm{~mm})$ & 17.25 & 8.63 & 6.31 & 5.75 & 4.31 \\
\hline $50 \mathrm{D}(20 \mathrm{~mm})$ & 13.80 & 6.90 & 5.05 & 4.60 & 3.45 \\
\hline \multirow[t]{2}{*}{$60 \mathrm{D}(16.66 \mathrm{~mm})$} & 11.50 & 5.75 & 4.21 & 3.83 & 2.87 \\
\hline & $\begin{array}{c}\text { Needed fg if } \\
\mathrm{Vf}=25\end{array}$ & $\begin{array}{c}\text { Needed fg if } \\
\text { Vf }=30\end{array}$ & $\begin{array}{c}\text { Needed fg if } \\
\text { Vf }=35\end{array}$ & $\begin{array}{c}\text { Needed fg if } \\
\text { Vf }=40\end{array}$ & $\begin{array}{c}\text { Needed fg if } \\
V f=45\end{array}$ \\
\hline $1 \mathrm{D}(1000 \mathrm{~mm})$ & 138.00 & 115.00 & 98.57 & 86.25 & 76.67 \\
\hline $2.0 \mathrm{D}(500 \mathrm{~mm})$ & 69.00 & 57.50 & 49.29 & 43.13 & 38.33 \\
\hline $2.5 \mathrm{D}(400 \mathrm{~mm})$ & 55.20 & 46.00 & 39.43 & 34.50 & 30.67 \\
\hline $4.0 \mathrm{D}(250 \mathrm{~mm})$ & 34.50 & 28.75 & 24.64 & 21.56 & 19.17 \\
\hline $5.0 \mathrm{D}(200 \mathrm{~mm})$ & 27.60 & 23.00 & 19.71 & 17.25 & 15.33 \\
\hline $7.5 \mathrm{D}(133.33 \mathrm{~mm})$ & 18.40 & 15.33 & 13.14 & 11.50 & 10.22 \\
\hline $10 \mathrm{D}(100 \mathrm{~mm})$ & 13.80 & 11.50 & 9.86 & 8.63 & 7.67 \\
\hline $15 \mathrm{D}(66.66 \mathrm{~mm})$ & 9.20 & 7.67 & 6.57 & 5.75 & 5.11 \\
\hline $20 \mathrm{D}(50 \mathrm{~mm})$ & 6.90 & 5.75 & 4.93 & 4.31 & 3.83 \\
\hline $25 \mathrm{D}(40 \mathrm{~mm})$ & 5.52 & 4.60 & 3.94 & 3.45 & 3.07 \\
\hline $30 \mathrm{D}(33.333 \mathrm{~mm})$ & 4.60 & 3.83 & 3.29 & 2.87 & 2.56 \\
\hline $35 \mathrm{D}(28.57 \mathrm{~mm})$ & 3.94 & 3.29 & 2.82 & 2.46 & 2.19 \\
\hline $40 \mathrm{D}(25 \mathrm{~mm})$ & 3.45 & 2.88 & 2.46 & 2.16 & 1.92 \\
\hline $50 \mathrm{D}(20 \mathrm{~mm})$ & 2.76 & 2.30 & 1.97 & 1.73 & 1.53 \\
\hline \multirow[t]{2}{*}{$60 \mathrm{D}(16.66 \mathrm{~mm})$} & 2.30 & 1.92 & 1.64 & 1.44 & 1.28 \\
\hline & $\begin{array}{l}\text { Needed fg if } \\
\text { Vf }=50\end{array}$ & $\begin{array}{l}\text { Needed fg if } \\
\text { Vf }=55\end{array}$ & $\begin{array}{c}\text { Needed fg if } \\
\text { Vf }=60\end{array}$ & $\begin{array}{c}\text { Needed fg if } \\
V f=65\end{array}$ & $\begin{array}{l}\text { Needed fg } \\
\text { if } \mathrm{Vf}=70\end{array}$ \\
\hline $1 \mathrm{D}(1000 \mathrm{~mm})$ & 69.00 & 62.73 & 57.50 & 53.08 & 49.29 \\
\hline $2.0 \mathrm{D}(500 \mathrm{~mm})$ & 34.50 & 31.36 & 28.75 & 26.54 & 28.75 \\
\hline $2.5 \mathrm{D}(400 \mathrm{~mm})$ & 27.60 & 25.09 & 23.00 & 21.23 & 19.71 \\
\hline $4.0 \mathrm{D}(250 \mathrm{~mm})$ & 17.25 & 15.68 & 14.38 & 13.27 & 12.32 \\
\hline $5.0 \mathrm{D}(200 \mathrm{~mm})$ & 13.80 & 12.55 & 11.50 & 10.62 & 9.86 \\
\hline $7.5 \mathrm{D}(133.33 \mathrm{~mm})$ & 9.20 & 8.36 & 7.67 & 7.08 & 6.57 \\
\hline $10 \mathrm{D}(100 \mathrm{~mm})$ & 6.90 & 6.27 & 5.75 & 5.31 & 4.93 \\
\hline $15 \mathrm{D}(66.66 \mathrm{~mm})$ & 4.60 & 4.18 & 3.83 & 3.54 & 3.29 \\
\hline $20 \mathrm{D}(50 \mathrm{~mm})$ & 3.45 & 3.14 & 2.88 & 2.65 & 2.46 \\
\hline $25 \mathrm{D}(40 \mathrm{~mm})$ & 2.76 & 2.51 & 2.30 & 2.12 & 1.97 \\
\hline $30 \mathrm{D}(33.333 \mathrm{~mm})$ & 2.30 & 2.09 & 1.92 & 1.77 & 1.64 \\
\hline $35 \mathrm{D}(28.57 \mathrm{~mm})$ & 1.97 & 1.79 & 1.64 & 1.52 & 1.41 \\
\hline $40 \mathrm{D}(25 \mathrm{~mm})$ & 1.73 & 1.57 & 1.44 & 1.33 & 1.23 \\
\hline $50 \mathrm{D}(20 \mathrm{~mm})$ & 1.38 & 1.25 & 1.15 & 1.06 & 0.99 \\
\hline $60 \mathrm{D}(16.66 \mathrm{~mm})$ & 1.15 & 1.05 & 0.96 & 0.88 & 0.82 \\
\hline
\end{tabular}

De-ionized (DI) water was first used to characterize the fluidic lens' radius of curvature range. DI water has an Abbe number of 55.74 and the indices of refraction are known for a wide scope of wavelength ranges [13]. The focal lengths of the DI water fluidic lens were first measured using red (HeNe $633 \mathrm{~nm}$ ), green $(\mathrm{HeNe} 543 \mathrm{~nm}$ ), and blue (Argon $488 \mathrm{~nm}$ ) lasers with the previously described pump controls. All three laser beams were aligned to the optical axis. The combination of the lasers allows to either individually test the lenses with specific wavelengths or to concurrently test multiple wavelengths as shown in Figure 2. The dichroic mirrors have specified bands in which they reflect and transmit. We found the proper dichroic mirrors to pass the previous wavelengths while reflecting the incoming perpendicular laser wavelengths. By mixing the lasers or adding an aperture stop, we varied the desired input wavelengths. 
We then used a single beam expander using an achromatic objective lens and another achromatic collimating lens to collimate the three beams in this single optical axis. A color CCD camera on a rail was employed to find the best focus spot. The spots were not of a perfect sphere since there was a slight amount of astigmatism observed at the focal spot. This is caused by a slight distribution in tension around the flanges and it is observed more drastically at longer focal lengths.

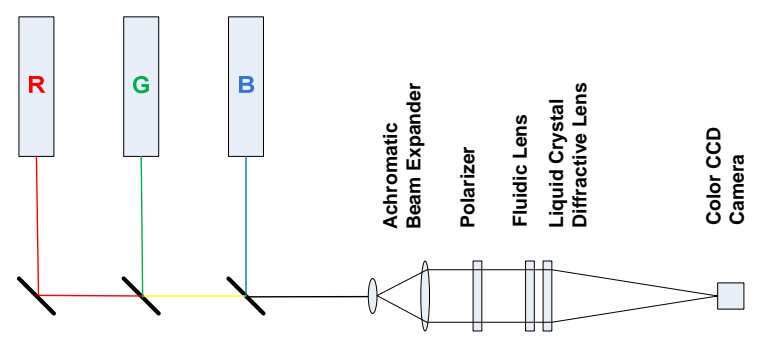

Fig. 2. The test setup: three laser beams aligned and collimated to measure the focal lengths of the diffractive and fluidic lens by a color CCD camera on a rail.

By identifying the distance from the fluidic lens, we measured the focal locations of lens at a specified amount of fluid with selected wavelengths. Radius of curvature, $r$, was determined from the focal lengths, $f$, of the DI water lens by noting the surface power $\Phi=1 / f=(n '-n) / r$. The radius of curvature was calculated for red, green, and blue wavelengths and averaged. It was found that the differentiation of the radii of curvature from the three wavelengths was from $0.02 \%$ to $0.8 \%$ for every specified amount of fluid pumped into the chamber, showing high repeatability. This plano-convex fluidic lens produced this accuracy for radius of curvature from $20 \mathrm{~mm}$ to $100 \mathrm{~mm}$, corresponding to a focal length range of $60 \mathrm{~mm}$ to $300 \mathrm{~mm}$ for DI water. Higher radii of curvature are achievable, where we tested to as high as $300 \mathrm{~mm}$ radius of curvature, equating to a focal length of $900 \mathrm{~mm}$ for DI water. Focal lengths around $600 \mathrm{~mm}$ to $900 \mathrm{~mm}$ output an accuracy variation between 2 and $5 \%$ of the expected focal spot. Thus, we are able to achieve focal lengths above $60 \mathrm{~mm}$ with DI water and know the physical range of the radii of curvature to be from $20 \mathrm{~mm}$ and up for this fluidic lens.

Table 1 identifies which Abbe number we would need the desired fluid to be with focal lengths in the relative area of $50 \mathrm{~mm}$ or higher. A value of $50 \mathrm{~mm}$ was chosen since selecting the fluid's index of refraction would permit for a slight variation in the focal length control. It is seen within the table that DI water would not be the fluid of choice since only one achromat focus is achievable with an Abbe number of 55. A fluid with an Abbe number of 15 would achromatize four of the diffractive lens' focal lengths and an Abbe number of 10 would achromatize five focal lengths. Theoretically, a fluid with an Abbe number of 5, as observed from Table 1, would produce approximately all possible achromatic combinations from either diffractive lens developed here. Thus, finding a fluid with known indices of refraction and characterized Abbe numbers from 5 to 15 offers the capability of a variable focal length achromat.

Methanol (Methyl alcohol) was chosen for the fluidic lens due to its high dispersion value and non-reactivity with the PDMS membrane. Methanol has an Abbe number of 13.66 and is widely available as it is a cleaning agent. In this case, the required focal length range of the fluidic lens would be from 16.8 to $101.0 \mathrm{~mm}$ in use with the diffractive lens B and from 63.14 to $252.6 \mathrm{~mm}$ in use with the diffractive lens A. As was previously stated, the diffractive lens A provides focal lengths of 1000,500 , and $250 \mathrm{~mm}$, and the diffractive lens B provides 400, 200, 133, 100, and $67 \mathrm{~mm}$ focal lengths. As seen from Table 1, all three possible focal lengths of the diffractive lens A, and two out of five focal lengths of the diffractive lens B (400 and 200) can be achromatized. Using Table 1, we identify the focal lengths of the fluidic lens for every focal length of the diffractive lens. Results of the two combined focal lengths develops a predicted achromatic focal length at green wavelength through $\Phi_{\text {expected }}=\Phi_{\text {diffractive }}+\Phi_{\text {fluidic }}$ and $f_{\text {expected }}=1 / \Phi_{\text {expected }}$. The five expected achromatic focal lengths, $f_{\text {expected}}$, for green must be achieved through the experimental setup for the achromat to work properly.

Focal lengths of the diffractive and fluidic lenses were first measured separately using the red $(\mathrm{HeNe} 633 \mathrm{~nm})$, green $(\mathrm{HeNe} 543 \mathrm{~nm})$, and blue (Argon $488 \mathrm{~nm}$ ) lasers. We used a linear polarizer with 
the diffractive lens to account for the polarization effects of the nematic liquid crystal. We can remove the polarizer if we add another liquid crystal diffractive lens with an orthogonal alignment to the first diffractive lens. As expected, the red light comes into focus first for the diffractive lens since it has negative dispersion. The test results for the diffractive lens are shown in Figure 3. The fluidic lens illustrates a nonlinear response as fluid is withdrawn. As the membrane reaches higher radii, the membrane becomes flatter. The sensitivity to the amount of fluid increases within this range since a smaller amount of fluid varies the curvature. The measured values of the fluidic lens focal length for the three wavelengths are depicted in Figure 4 in terms of the amount of fluid injected in the lens.

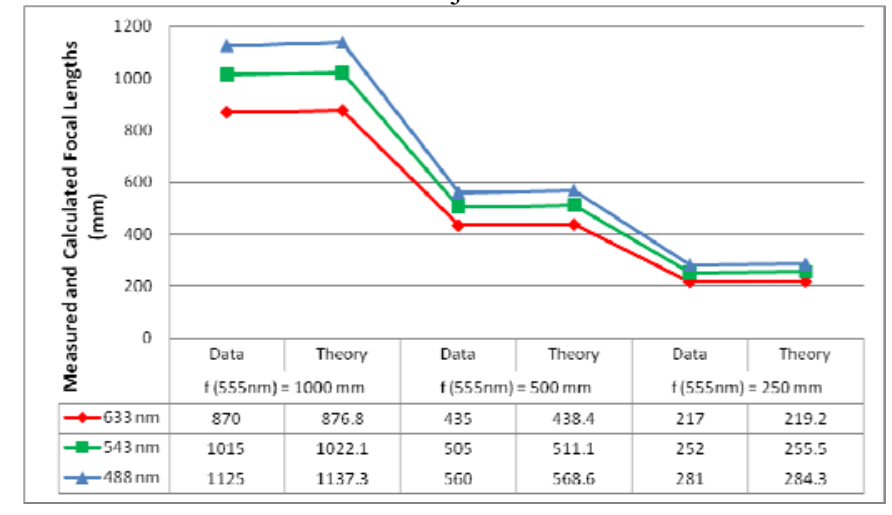

(a)

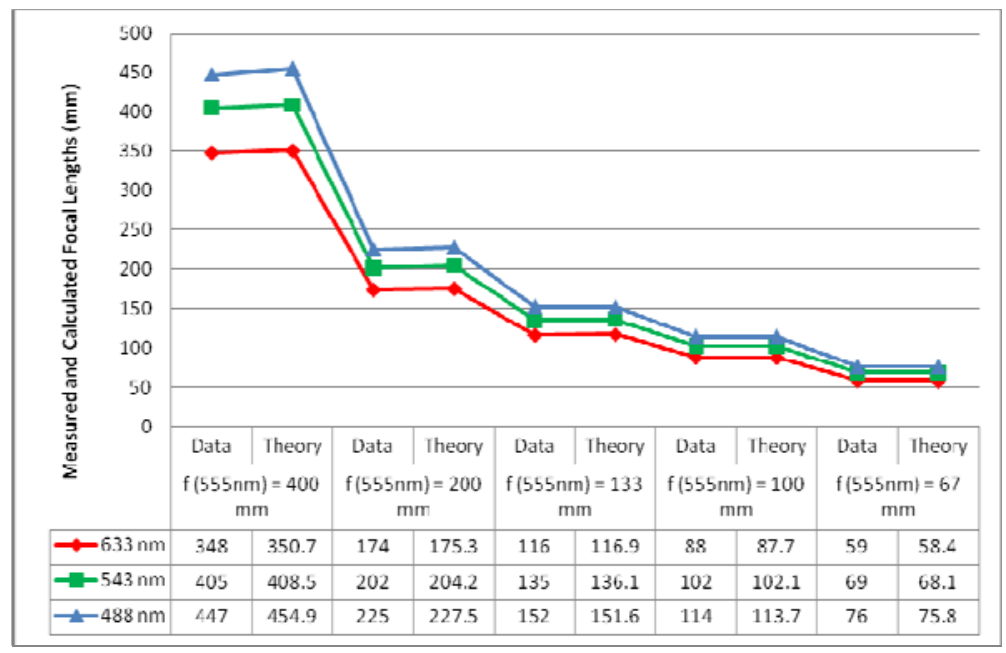

(b)
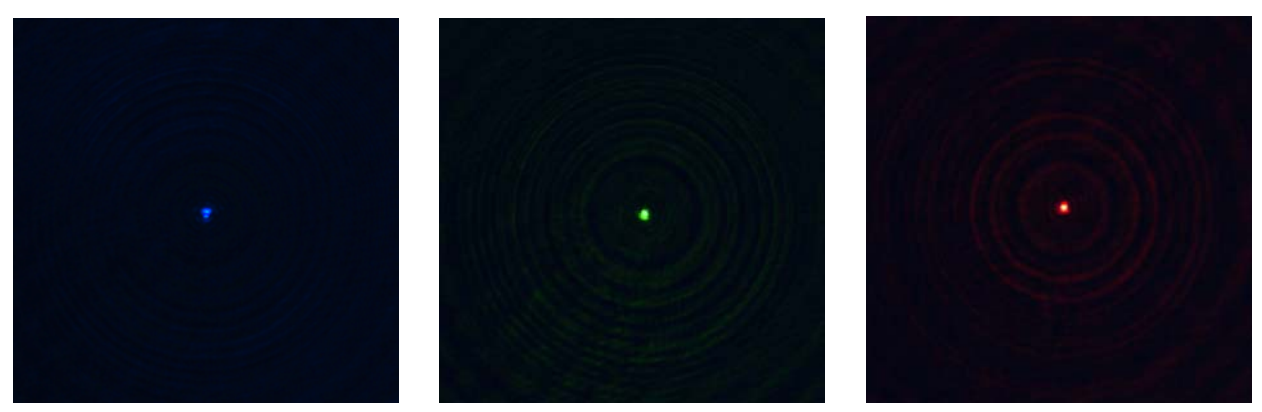

(c) 
Fig. 3. Diffractive lens results: measured and calculated focal lengths at the three test wavelengths versus the design focal length at $555 \mathrm{~nm}$ for the (a) diffractive lens A and (b) diffractive lens B. (c) Sample images of the best focus spot for the diffractive lens B when it is set to the focal length of $133 \mathrm{~mm}$.

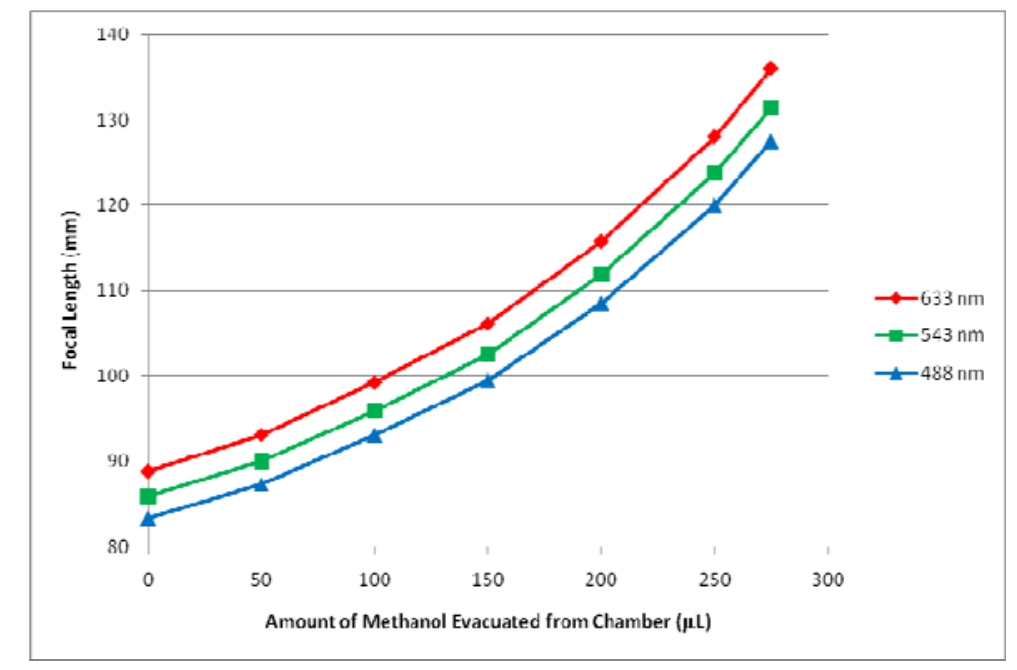

Fig. 4. Chromatic dispersion of variable focal plano-convex lens applying methanol at the three test wavelengths when set for $80 \mathrm{~mm}$ focal length and higher.

The final step in producing the variable focal length achromat is the combination of the liquid crystal diffractive lens with the pressure controlled methanol fluidic lens. After adjusting the focal lengths of each lens to the appropriate values dictated by the achromat equation we measured the overall focal length of the hybrid lens at the red, green, and blue wavelengths. The experiment verified that the focus spots of the red and blue wavelengths coincided very closely. Figure 5 (a-e) depict the focal spots for the red and the blue wavelengths as the focal lengths of the diffractive and the fluidic lenses are varied according to the achromat equation. As the focal length is decreased, the spot size and aberrations are reduced as expected; however, the background scattered light is slightly increased. This is caused by the reduction in diffraction efficiency at the shorter focal lengths as the number of binary phase levels decreases [2, 3]. Figure 5(f) shows the values of the overall focal length of the hybrid diffractive/fluidic lens for the green, blue and red test wavelengths. As expected, the green light comes to focus first and then the red and blue lights will come into focus at the same plane. The measured focal lengths of the hybrid lens closely matched with the expected focal length values seen from figure 5(f). 

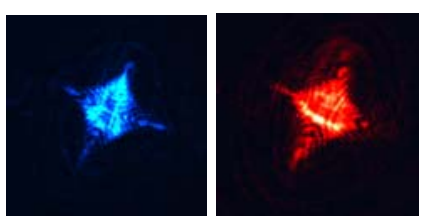

(a)
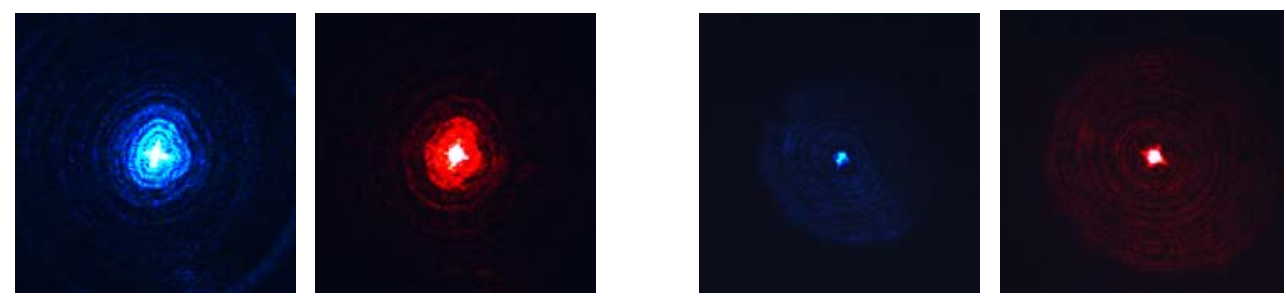

(d)

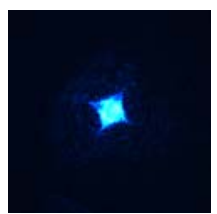

(b)

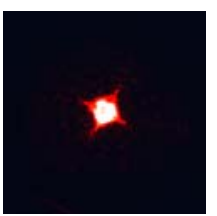

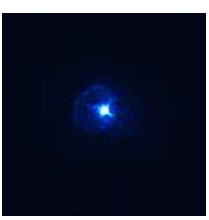

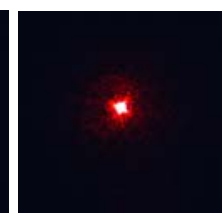

(c)

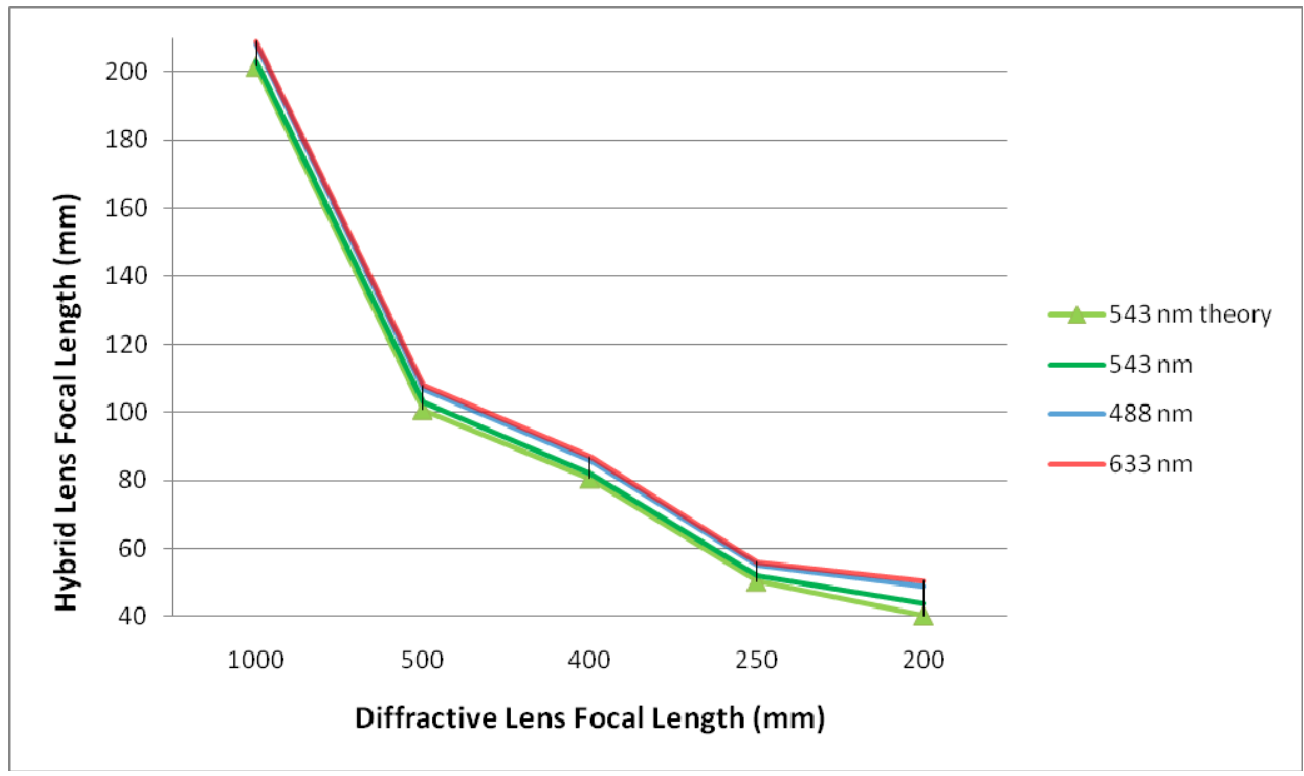

(f)

Fig. 5. Focal spots when the diffractive and fluidic lenses are combined to produce the best focus for the red and the blue lights, when at the green wavelength: (a) $\mathrm{f}_{\text {diffractive }}=1000 \mathrm{~mm}, \mathrm{f}_{\text {fluidic }}=252 \mathrm{~mm} ;(\mathrm{b}) \mathrm{f}_{\text {diffractive }}=500 \mathrm{~mm}, \mathrm{f}_{\text {fluidic }}=126 \mathrm{~mm} ;(\mathrm{c}) \mathrm{f}_{\text {diffractive }}=250 \mathrm{~mm}, \mathrm{f}_{\text {fluidic }}$ $=63 \mathrm{~mm} ;(\mathrm{d}) \mathrm{f}_{\text {diffractive }}=400 \mathrm{~mm}, \mathrm{f}_{\text {fluidic }}=101 \mathrm{~mm}$; (e) $\mathrm{f}_{\text {diffractive }}=200 \mathrm{~mm}, \mathrm{f}_{\text {fluidic }}=51 \mathrm{~mm}$; (f) overall focal length of the hybrid system for the green, red and blue wavelengths.

In conclusion, we have demonstrated a variable focal length achromatic lens that consists of a variable liquid crystal diffractive lens and a variable pressure-controlled fluidic lens. We used two diffractive lenses that produce multiple discrete focal lengths with an Abbe number of -3.45 . The fluidic lens can provide a more continuous variation, and its focal lengths are chosen such that they correct for the dispersion of the diffractive lens. We chose Methanol for the fluidic lens due to its high dispersion properties. Then we combined the fluidic lens and one diffractive lens at a time to minimize the dispersion between the red and blue wavelengths. The lenses showed acceptable optical properties and the test results were close to the theoretical predictions. This adjustable hybrid lens has no moving parts and would be useful for compact color imaging applications, and medical and ophthalmic imaging devices. 


\section{APPENDIX G}

\section{FABRICATION PROCEDURE}

Follow this procedure for cleaning the 4 inch wide octagonal shaped ITO coated glass slides.

1. First make sure there are no loose glass pieces on the wafer left from scribing and cutting procedure by rinsing the wafer under DI water.

2. Using ultralow particulate wipe, scrub the wafer under running DI water for 1-3 minutes. Make sure all areas of the wafer have been adequately scrubbed.

3. Place the wafer in a Petri dish and cover it with acetone. Scrub it with a clean low ultralow particulate wipe for 1-3 minutes. Rinse the wafer with acetone.

4. Cover the wafer with Isopropyl alcohol in a Petri dish and scrub it for at least one minute. Rinse it with IPA.

5. Rinse the wafer with water for 30 seconds. Rinse it with clean, no impurity IPA.

6. Use nitrogen to dry the wafer. Start drying it from the center and proceed to the edge.

7. Examine the wafer under microscope. Usually the wafer is clean enough after these steps. 


\section{PRIMING THE WAFERS:}

1. Tape the wafer to the cover of a Petri dish, using Capton tape. Avoid attaching the tape to any part of the wafer. Cover the part of the tape which sits on the wafer with aluminum foil so that only the foil is in touch with wafer.

2. Using a clean syringe draw $0.5 \mathrm{ml}$ HMDS, inject it in a Petri dish on a hot-plate at $60 \mathrm{C}$, and place the cover with taped wafer on it. The HMDS will evaporate from the bottom and condense on the slide after about 5-10 minutes.

3. After 5-10 minutes, make sure the surface of the wafer is completely covered with HMDS, and place the cover on a hot-plate at 120C and bake the coating for 3-5 minutes.

4. Make sure the wafer is cover at all times so that no particulate will be added and stuck on the surface.

5. All procedures must be performed under hood and good ventilation system. HMDS is carcinogenic.

6. Non-uniform coating of HMDS can result in a non-uniform coating of photoresist. Before spin coating photoresist, the wafer must be treated in oxygen plasma for 30 seconds. 


\section{SPIN COATING PROCEDURE:}

1. Heat the wafer on a hot place at $200 \mathrm{C}$ at least for 5 minutes if the wafer is not primed. Remove the wafer from the hot plate and allow 2 minutes to cool down to room temperature.

2. If the wafer is primed, take the wafer out of plasma, and wait for 30 seconds before coving it with photoresist.

3. Place the wafer on the 4 inch chuck on the spinner, and dispense dilute photoresist on the wafer. Use a solution of 2 parts Shipley 1805 and 1 part propylene glycol monomethyl ether acetate. Use a 0.45 PTFE filter to remove particulates in the mixture. Cover the wafer with the mixture thoroughly.

4. Wait for 2 minutes.

5. Use these parameters for the spin coater recipe: lid: closed, maximum speed: 2500RPM, ramp: 500RPM/sec, spinning time: 15 seconds. The whole procedure is going to take about 25 seconds because 15 seconds only refers to the time that the spinner is rotating at the maximum speed. It takes 5 seconds to reach that speed and 5 seconds to slow down to zero.

6. Place the coated wafer on a hotplate at $115 \mathrm{C}$ for 3 minutes. Notice while removing the wafer from the spinner solvent evaporation from the coated film creates a temporary fringe pattern that disappears in seconds. The coated film should be uniform and look pinkish for this speed. This procedure results in a thin film usually thinner than 200nm. 


\section{PATTERNING THE ELECTRODES:}

Electrode patterning includes the following steps:

1. Cutting ITO coated sheets.

2. Cleaning the wafers.

3. Coating a thin film of $\mathrm{SiO} 2$ on the ITO layer (50nm). [Ask Olli to do it]

4. Coating a thin film of chromium $(50 \mathrm{~nm})$ on $\mathrm{SiO} 2$ layer. [Use e-beam evaporator]

5. Priming chromium coated wafer.

6. Spin coating photoresist on the wafer and prebaking the photoresist.

7. Exposing the baked photoresist film to UV light.

8. Developing the patterned photoresist.

9. Hard-baking the photoresist.

10. Etching chromium.

11. Etching $\mathrm{SiO} 2$.

12. Etching ITO.

13. Stripping the photoresist.

14. Etching chromium. 


\section{ASSEMBLY OF THE LIQUID CRYSTAL CELL}

1. Do a final inspection and cleaning of 1 patterned substrate and 1 unpatterned substrate as A2 to remove ALL macroscopic contaminants.

2. Mask off the appropriate electrical contacts on each substrate with Kapton tape.

3. Spin coat alignment layer. For Formic acid, step 1: sit $30 \mathrm{sec}$; Step 2: $60 \mathrm{sec} @$ 2000 rpm. For nylon + formic mix, Step 1: sit 60 s; Step 2: 120 sec @2000 rpm.

4. Bake the alignment layer in the oven $\left(90 \min @ 115^{\circ} \mathrm{C}\right)$.

5. Buff each substrate in a single direction as shown in the figure and record the direction with a marker on the opposite side of the substrate. This is to ensure that when the cell is assembled the rub directions are parallel, but pointing in opposite directions (the "anti-parallel” configuration).

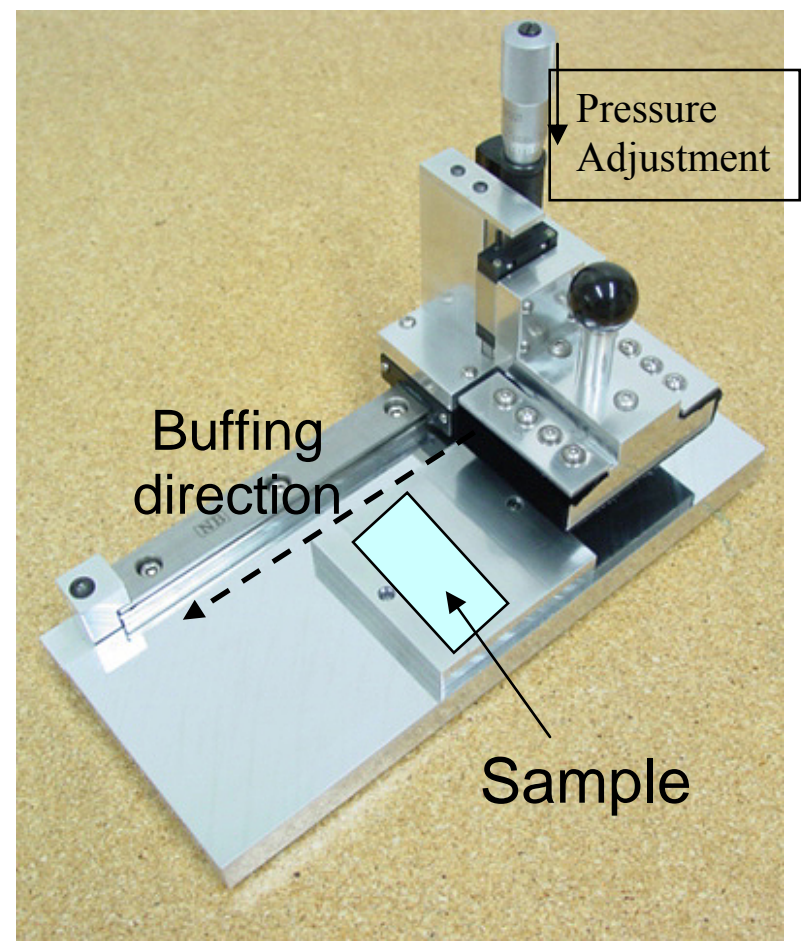


6. Load the reference electrode into the assembly jig, alignment layer up such that it is flush to the jig along the long edge and held firmly in place.

7. Disperse 5- $\mu \mathrm{m}$ spacers in Ethanol onto the alignment layer of the patterned, square substrate. (Put a few drops of the spacers and run the spinner for $30 \mathrm{sec} @$ 3000 rpm.)

8. Place 4 SMALL drops of the NOA65/spacer mixture at the edges of the reference back plane where it intersects the edges of the patterned substrate. Be careful that the spacers dispersed across the substrate and used in the NOA65 adhesive are the same size.

9. CAREFULLY place the patterned substrate onto the reference substrate, making sure the alignment layers are adjacent to one another and that the rub directions are in the anti-parallel configuration. Also make sure the patterned substrate is flush with the jig.

10. Use tweezers to compress and slightly shear the cell. Use the observed interference fringes as a guide; when there are only a few fringes observed across the overlapping area the cell can be considered to be only 1 spacer thickness thick.

11. CAREFULLY remove the assembled cell from the assembly jig and expose under the mask aligner for 5 minutes to cure the NOA65 adhesive. 


\section{FILLING IN THE LIQUID CRYSTAL:}

1. Place the empty cell on a $75^{\circ} \mathrm{C}$ digital hot plate and let the cell come to temperature.

2. Using a sealed glass capillary tube place a few drops of E7 at the gap where the two substrates meet and allow capillary action to draw the liquid crystal into and fill the cell.

3. Hold the cell at $75^{\circ} \mathrm{C}$ for one hour and then cool at $30^{\circ} \mathrm{C} /$ hour to $30^{\circ} \mathrm{C}$.

4. After the cell has reached $30^{\circ} \mathrm{C}$ remove from the hot plate and wipe away any excess liquid crystal. The cell is ready for testing. 
APPENDIX H

A SAMPLE CODE FOR THE DESIGN AND SIMULATION OF A ZOOM LENS

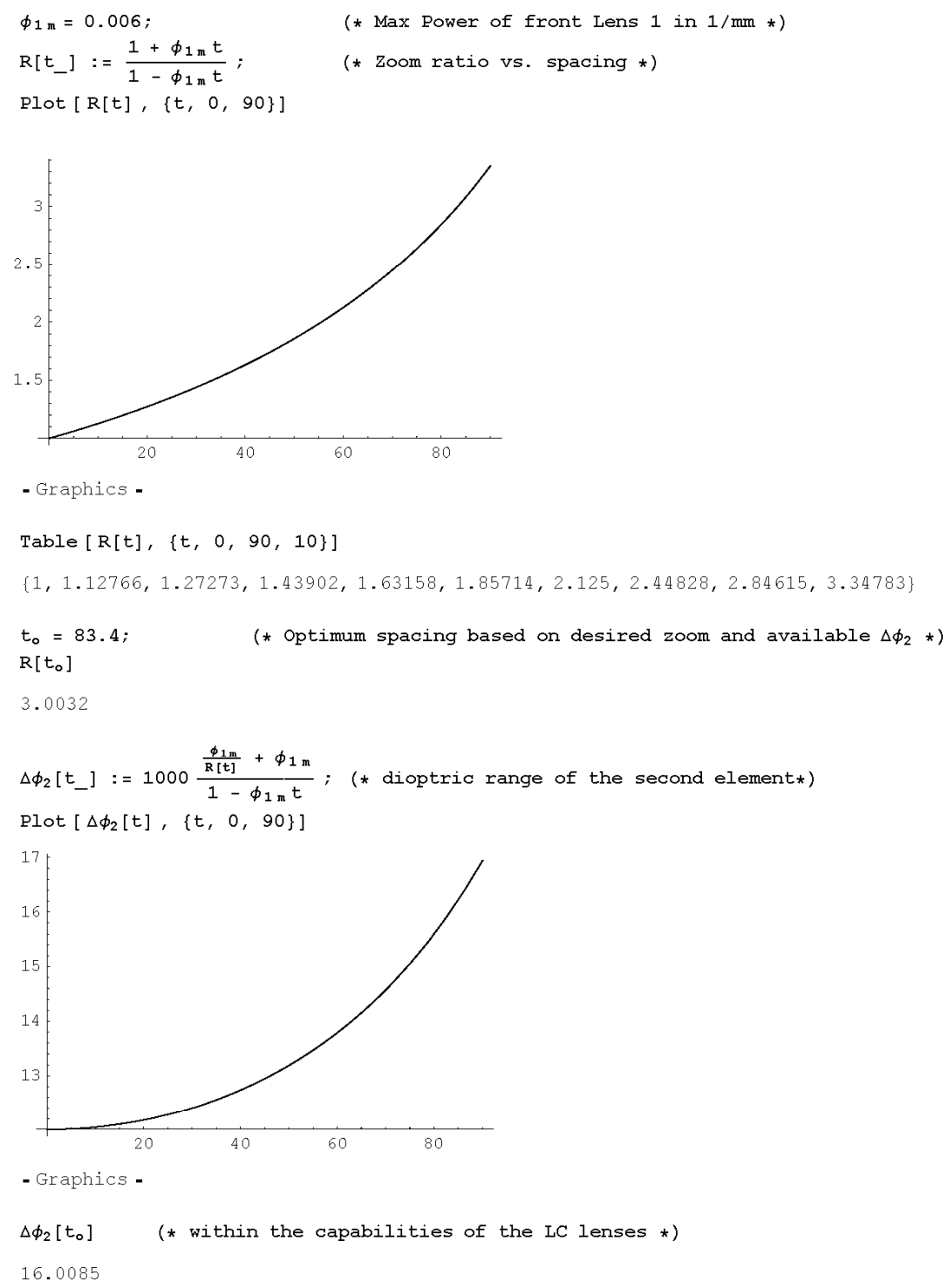




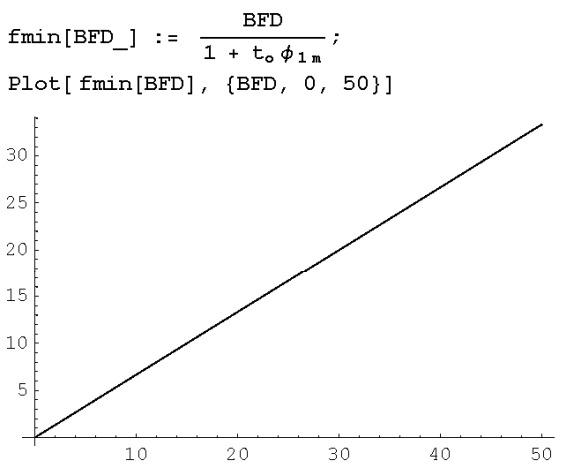

- Graphics -

$\operatorname{fmin}\left[9.614891201890389^{\prime}\right]$

6.40822

$\mathrm{f}_{2 \max }\left[\right.$ BFD_] $:=\frac{\left(1-t_{0} \phi_{1 \mathrm{~m}}\right) \text { BFD }}{1-\phi_{1 \mathrm{~m}}\left(t_{0}+\text { BFD }\right)}$

$\left(* f_{2 \max }=2\right.$ nd static lens focal length *)

Plot $\left[f_{2 \max }[B E D],\{B F D, 0,20\}\right]$

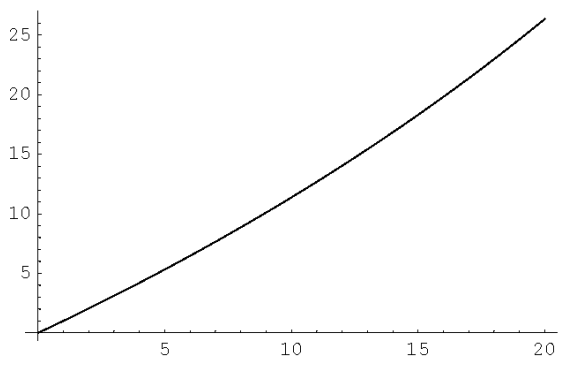

- Graphics -

$f_{\text {static }}=10 ;$

(* a standard static picked *)

$f_{\text {static } 2}=\frac{1}{\left(\frac{1}{f_{\text {static }}}-\frac{0.5 \Delta \phi_{2}\left[t_{0}\right]}{1000}\right)}$

10.8701

(* BFD should be close to $t$ o to maximize $\Delta f$,

and to $f_{2 \max }=f_{\text {static2 }}$ to focus objects $\frac{f_{A C \min }}{2} \rightarrow \infty$ [Tam's paper] *) 


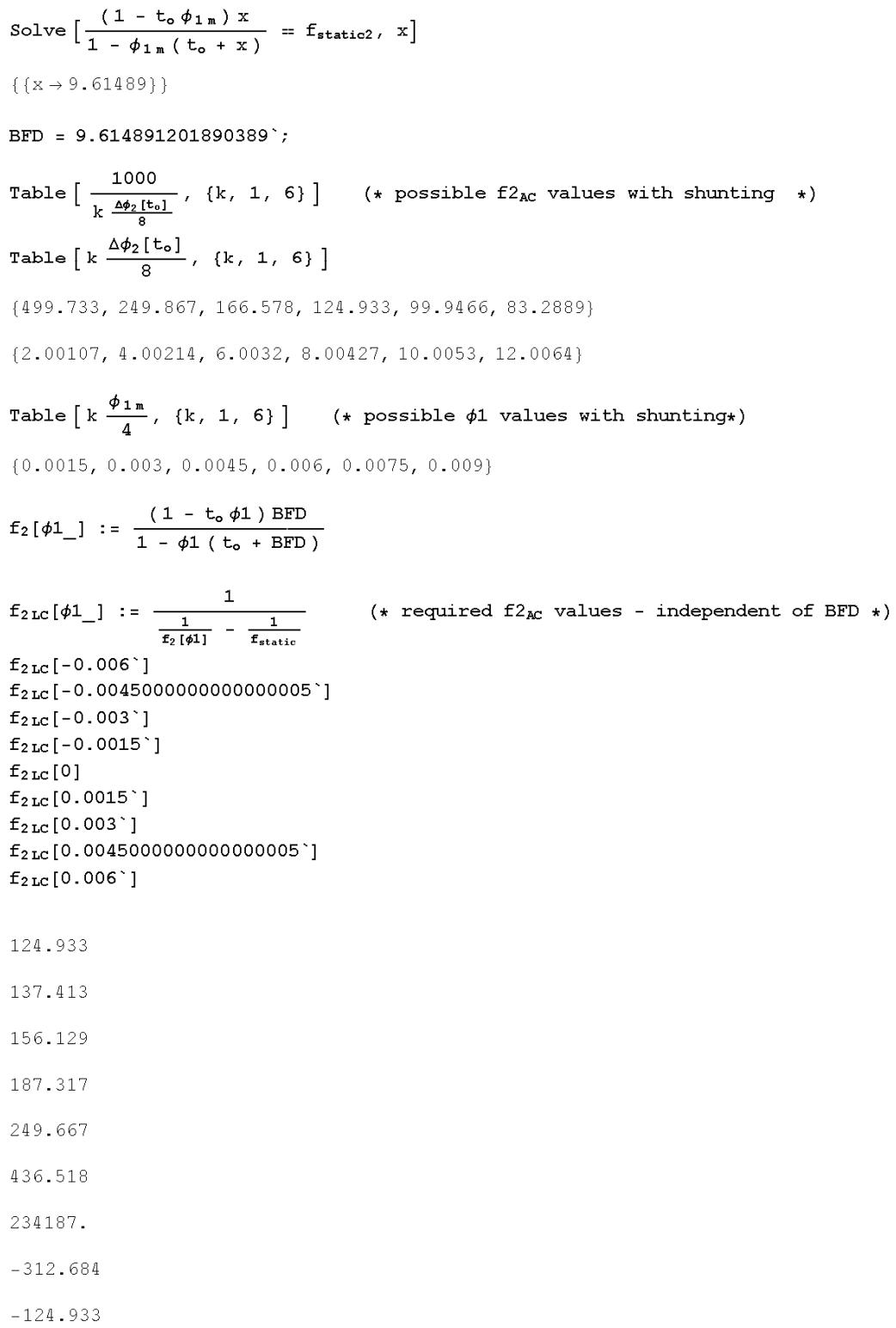




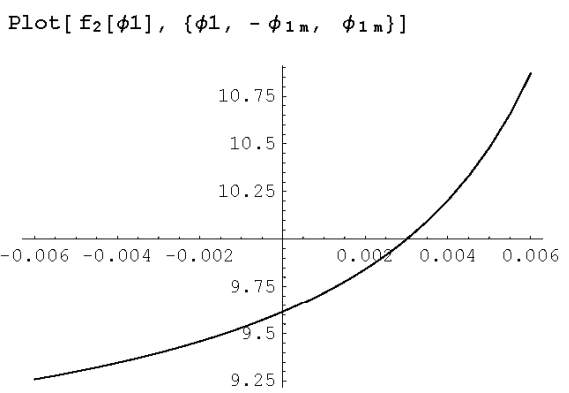

- Graphics -

$$
\begin{aligned}
& \phi\left[\phi 1 \_\right]:=\phi 1+\frac{1}{f_{2}[\phi 1]}-\phi 1 \frac{1}{f_{2}[\phi 1]} t_{0} \\
& f_{\text {total }}\left[\phi 1 \_\right]:=\frac{1}{\phi[\phi 1]} \\
& P \operatorname{Plot}\left[f_{\text {total }}[\phi 1],\left\{\phi 1,-\phi_{1 \mathrm{~m}}, \phi_{1 \mathrm{~m}}\right\}\right]
\end{aligned}
$$

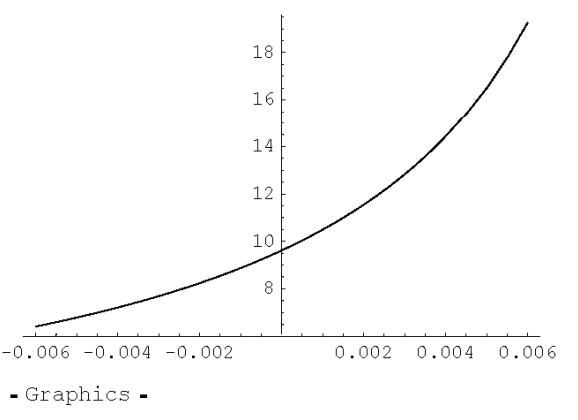

$f_{\text {total }}\left[-\phi_{1 \mathrm{n}}\right]$

6.40822

$f_{\text {total }}\left[\phi_{1 \mathrm{~m}}\right]$

19.2452

$f_{\text {total }}\left[\phi_{1 \mathrm{~m}}\right]$ $f_{\text {total }}\left[-\phi_{1 \mathrm{~m}}\right]$

(* Verification of the zoom ratio *)

3.0032

$f_{1 \mathrm{~m}}=\frac{1}{\phi_{1 \mathrm{~m}}}$

166.667 


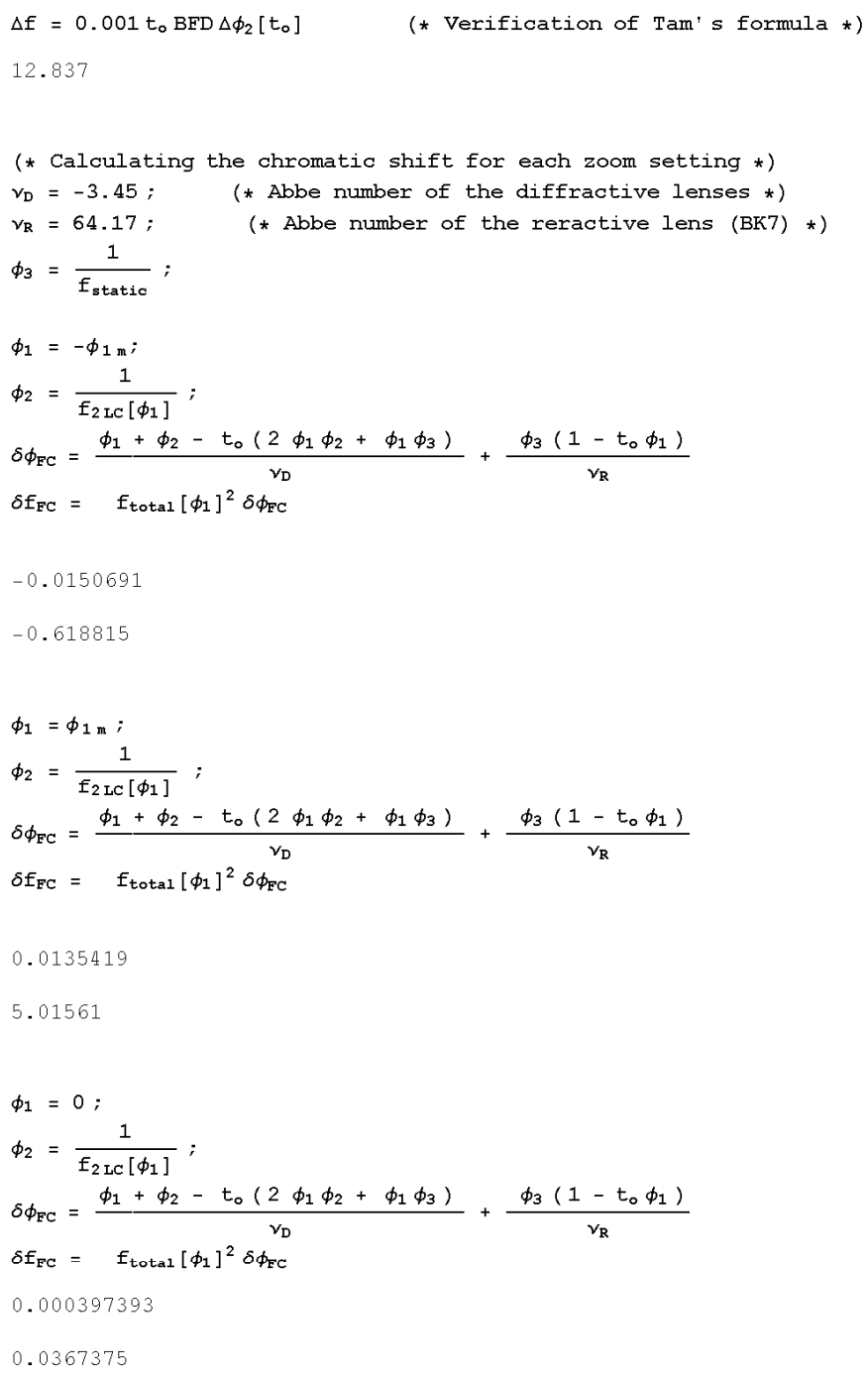




\section{APPENDIX I}

INNOVATIVE LENSES FOR CAMERA PHONES

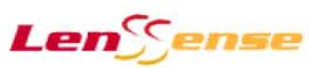

Innovative Lenses for Camera Phones

Submitted to

Nokia's Technology Innovation Competition

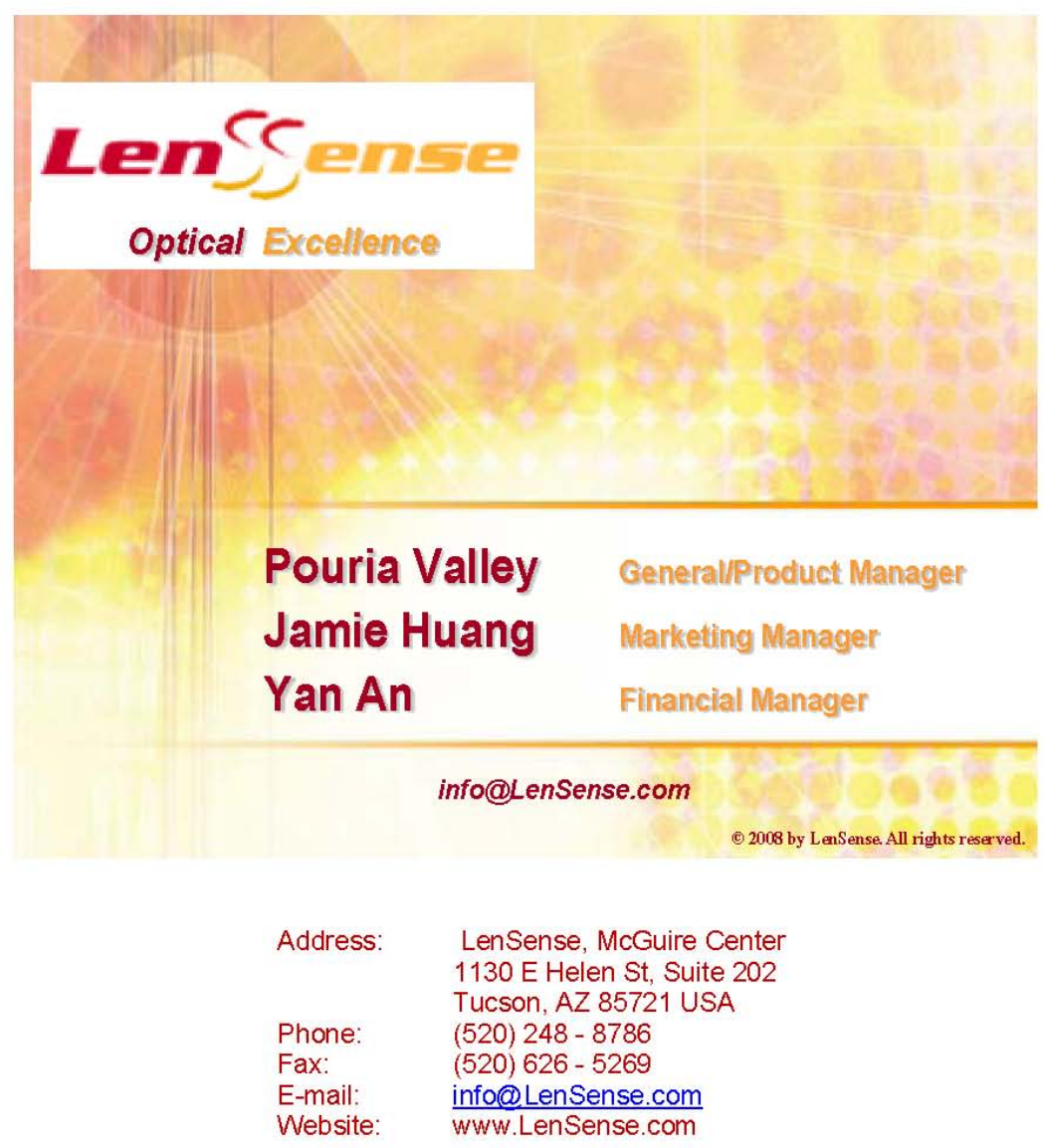

February 8, 2008 


\section{Lençense}

\section{Table of Contents}

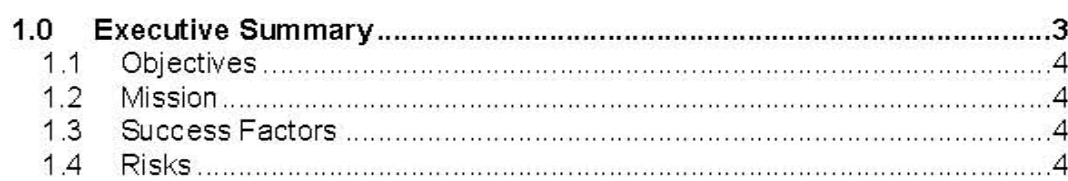

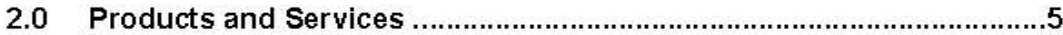

2.1 Products and Services Description .............................................

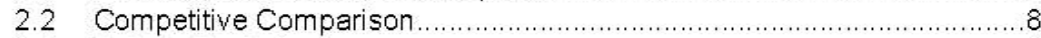

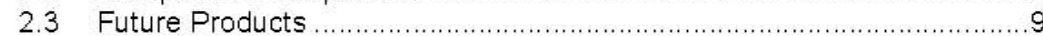

3.0 Market Analysis Summary ................................................................ 10

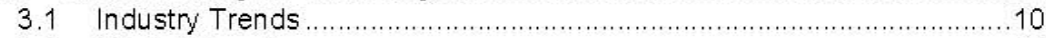

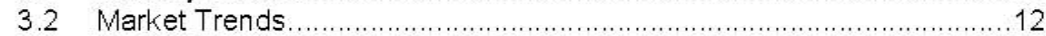

3.3 Competitive Advantage for Nokia ............................................ 13

4.0 Strategy and Implementation Summary ...........................................15

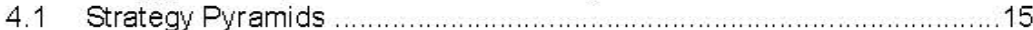

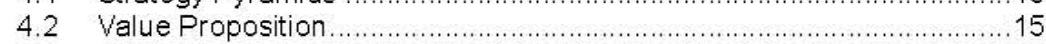

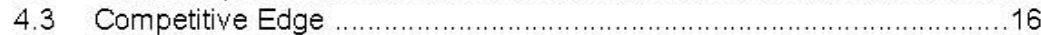

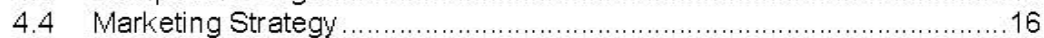

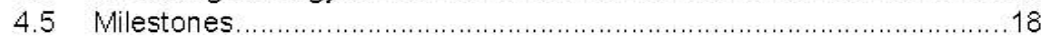

5.0 Management Summary ....................................................................19

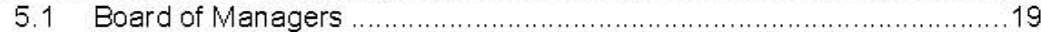

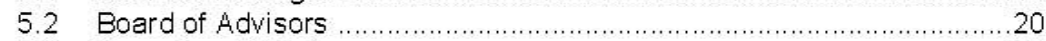

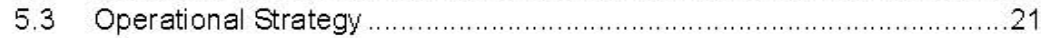

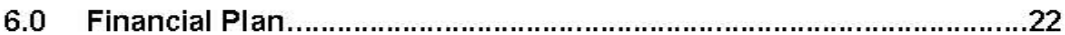

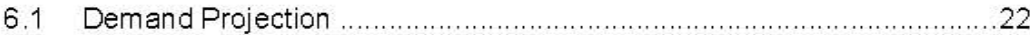

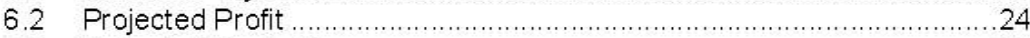

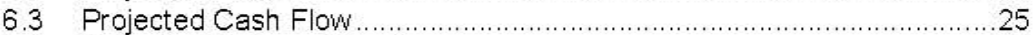

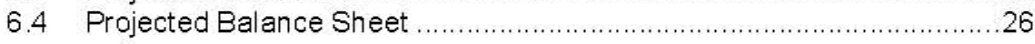

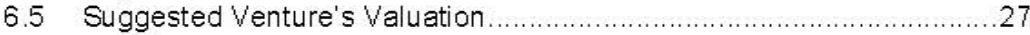

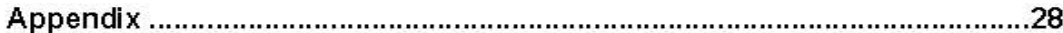

Appendix 1: Assumptions to Financial Statements:

Appendix 2: Proceedings of the National Academy of Sciences' Article ........29

Appendix 3: University of Arizona Alumnus Magazine's Article .....................34 


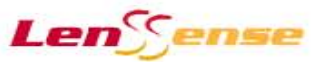

\subsection{Executive Summary}

Digital cameras are expected to become a standard feature in $90 \%$ of all new mobile phones by 2009 . $^{1}$ However, typical camera phones in use today lack optical zoom performance because they utilize only one lens with a fixed focal length. Moreover, the picture quality is acceptable only for capturing details in a wide area but not a deep one. Therefore, only a few subjects appear sharp in the image. Perhaps for this reason, $35 \%$ of all camera phone users believe the picture qualities are too poor to make prints. ${ }^{2}$

LenSense electro-active zoom lenses blend a proprietary mixture of optics, chemistry, and electronics invented at the world-renowned College of Optical Sciences, University of Arizona. This technology improves the picture quality and resolution of camera phones with lower operating voltage (longer battery life), faster response time, and more robustness at a competitive cost. In addition, LenSense will open up new aftermarket sales for a wide variety of potential new applications.

The LenSense target collaborative partner is Nokia (Espoo, Finland), the largest cell phone manufacturer in the world with net sales of EUR 41.1 billion in 2006 . According to a scientific paper published by Nokia, there are currently more than 500 million camera phone users worldwide. Of all new cell phones sold today, more than half incorporate an integrated camera. In addition, more than $75 \%$ of all the digital image capturing devices in use today are camera phones. The total sales volumes of all camera phones surpassed 600 million units per year by $2008^{3}$

The LenSense business model is focused on creating new, advanced lens technology R\&D and prototyping. The process of fabricating lenses is similar to that used in the semiconductor industry. Therefore, mass production can be outsourced to integrated circuit (IC) manufacturers at a low unit cost. The company will use automated assembly with high quality control machinery, and follow Nokia's Standard Mobile Imaging Architecture (SMIA) standard.

The goal of LenSense is to supply a minimum of $25 \%$ of camera phone lenses manufactured by Nokia. With projected annual growth rate between $15-20 \%$, sales are expected to reach $\$ 124$ million with EBITDA exceeding $\$ 40$ million by Year 5.

${ }^{1}$ Info Trends

${ }^{2} 2005$ PMA U.S. Consumer Photo Buying Report

${ }^{3}$ Proceedings of SPIE Vol. 6196, 61960B, 2006 


\section{Len'fense}

\subsection{Objectives}

LenSense will improve camera phone performance and image quality while simultaneously enhancing the customer's experience with point-n-shoot photography. The LenSense vision is to supply a lens into one of every four camera phones worldwide by 2013. These objectives will be initiated by taking the following steps:

Obtain collaborative contracts with Nokia

$\rightarrow$ Develop complete product lines in manufacturing lenses

\subsection{Mission}

The LenSense mission is to design and build the most advanced lenses for use in mobile phones throughout the world, by partnering with Nokia and a semiconductor manufacturer.

\subsection{Success Factors}

Acceptance of LenSense innovative technology as a significant improvement to the current lens solutions

Early entrance to expand the mobile market and explore new ones

$>$ Developing good relationships with our partners and customers

\subsection{Risks}

Underestimating unit sales: Although the sales have been projected conservatively, the true demand could be higher than forecast. In addition, the true cost of production is likely to fluctuate. The company should be prepared to face operational and managerial issues.

Time to market: Short time to market is the key to succeed in the mobile industry. Nokia used to take 12 to 18 months to develop their basic models a few years ago, compared to about 6 months nowadays.

Adopter categorization: It is difficult to anticipate how this revolutionary LenSense technology will be accepted by the mass market. By understanding the timing of adoption stages such as early majority, demand can better be forecasted. 


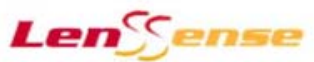

\subsection{Products and Services}

The initial LenSense product is a compact, voltage-controlled, zoom lens module with no moving parts. In contrast, traditional zoom lens systems are bulky, expensive, and require mechanical movements. Our lenses also improve the depth of field and image quality by providing multiple focal lengths based on low voltages applied to an electro-optic medium incorporated in the lens.

This innovative technology was invented at the University of Arizona's College of Optical Sciences, and developed successfully for vision care applications. (Please see appendix 2 for technical details). After securing intellectual properties, multiple scientific papers were published, and a full working prototype was built which received significant attention and coverage by the public media. (One example is attached in appendix 3 ). This technology prototype is currently on its way to become a commercial product by a vision care corporation for the specific ophthalmic applications. LenSense is further improving this technology to fit in numerous other applications including the camera phones.

\subsection{Products and Services Description}

Lens modules made by LenSense are comprised of two main parts: a diffractive element, and a thin layer of liquid crystal. The first step in making the diffractive element is to design it by Computer Aided Design (CAD) software like AutoCAD.

Figure 1 shows the top view of the first two zones of a diffractive pattern known as Fresnel zones. Each zone is divided into several subzones or rings.

Depending on the aperture size, there could be several hundreds or thousands of rings. The lines represent electrical bus bars that bring voltages to the subzones.

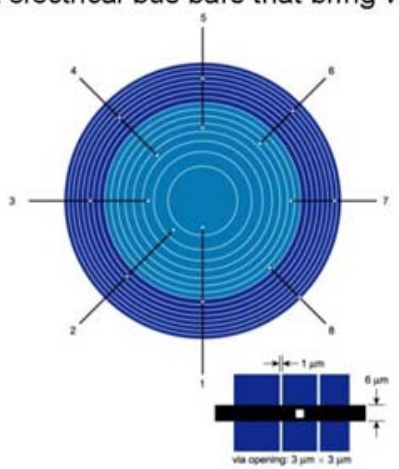

Figure 1: Top view of the diffractive pattern 


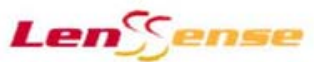

In the second step, the patterns are transferred and etched into the glass substrates pre-coated with thin layers of Indium-Tin-Oxide (ITO). The fabrication techniques of the diffractive patterns are very similar to those used in the semiconductor industry. These processes require high quality lithography equipments and tight environmental control in terms of contamination, temperature, humidity, etc. Therefore, the fabrication needs to be performed in a clean room of class 100 or better. Figure 2 shows one of the Class- 100 clean rooms at the University of Arizona.

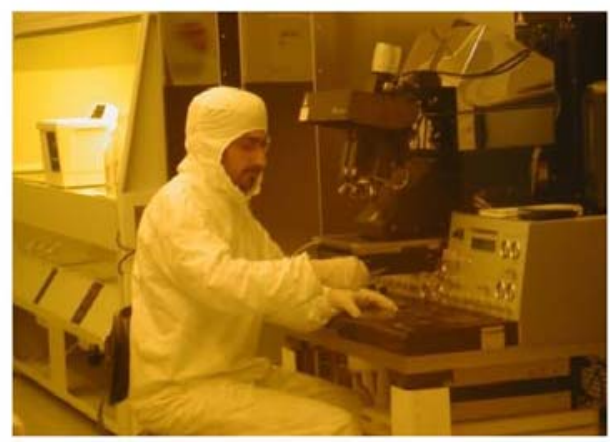

Figure 2: Pouria Valley uses MA6 exposure system in a class 100 clean room

In the final step, a thin layer of liquid crystal is filled in between two thin ITO glass substrates, one with the diffractive pattern and one with no pattern used as the electric ground (Figure 3 ). Finally, the lenses are wire-connected to a small electronic driver that feeds small amounts of voltages across the liquid crystal. Various tests can be performed to characterize the lenses. These include diffraction efficiency measurement, microscopy, imaging, and interferometry.

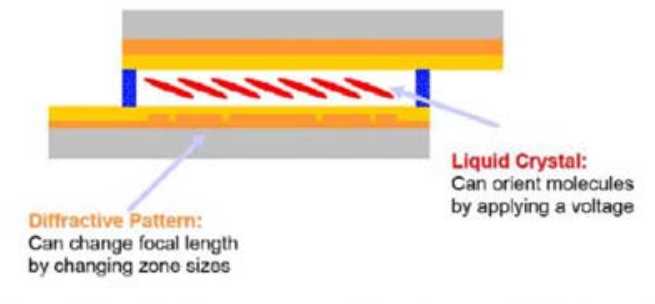

Figure 3: Simplified diagram of a LenSense lens module (side view) 


\section{Lencense}

Optical zoom is an important feature for camera systems, but is missing in almost all camera phones. Many camera phones offer digital zoom where the image is magnified, but the resolution is lost (Figure 4). With optical zoom, the resolution can actually be increased.

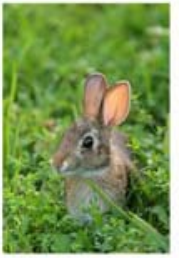

Original

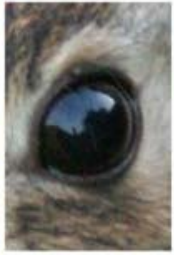

10x Optical

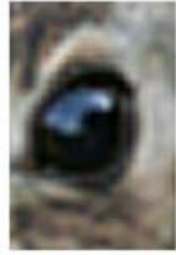

10x Digital

Figure 4: Comparison of optical vs. digital zoom [source: photo.net]

Because of the space constraints, most camera phones employ only one lens element with a fixed focal length. To achieve optical zoom, a system needs to have multiple focal lengths. The current solution is the multi-element zoom lens systems, such as those utilized in most digital still cameras and camcorders. Unfortunately, this solution is not suitable for cell phones because zoom lenses are relatively bulky and expensive.

Depth of field, the range of distance around a subject that appears acceptably sharp in image, is also affected by the lens focal length. Cameras with a shorter focal length result in a smaller depth of field for a given lens aperture, which is not desirable (Figure 5).

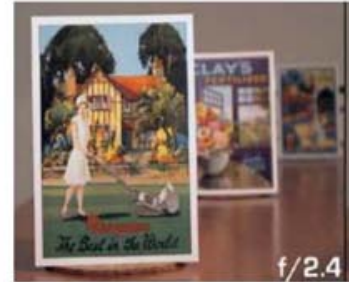

Blurry with current lens technology

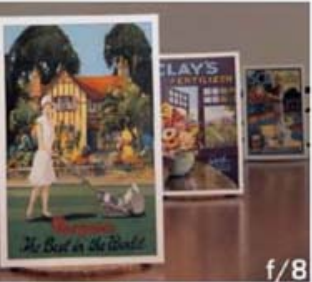

Sharp with LenSense

Figure 5: Depth of field concept: Three subjects were imaged using two different f-numbers. The picture on left was taken with a shorter focal length and so the last two subjects appear less sharp compared to the picture on the right. [Source: dpreview.com]

Also, due to the short focal length of cell phone lenses, the field of view is very wide. This means their image quality is acceptable for far subjects or wide angle shots, but is poor for near subjects or close-ups. 


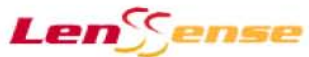

\subsection{Competitive Comparison}

The main competitors of the LenSense are VariOptic and Holochip. Their technologies are electro-wetting and Micro-Electro-Mechanical Systems (MEMS).

Varioptic produces liquid lenses with glass and plastic lenses. Varioptic is based in France, but has regional offices in Europe and Asia including Taiwan, Japan, and China. It has a global supply chain and focus primary on the Asian market. Varioptic is ready to get in the product stage but its lenses have not appeared in any prominent cell phone brands.

In the electro-wetting technique used by Varioptic, a lens cell is filled with two non-mixing fluids, one an electrically conductive liquid (like water) with the other being a non-conductive liquid (like oil). A variable voltage is applied to the liquids which causes a curvature change of the liquid-liquid interface that in turn leads to a change of the focal length.

Holochip on the other hand is developing adaptive polymer lenses. Holochip's headquarter is in New Mexico, U.S.A. and has one west regional office in California. This company is still in the prototype stage.

MEMS technique is based on micro-scale mechanical movements. A lens cell in this case comprises a membrane, chamber, periphery sealing ring, and a liquid with fixed volume. A servo motor is used to push in or pull out the liquid in the chamber resulting in the lens shape change and hence, variation of focal length.

The LenSense technology has significant advantages over the aforesaid technologies that are summarized in Table 1:

Table 1: LenSense competitive advantages

\begin{tabular}{|c|c|c|c|}
\hline Feature & Company & Len CS \\
\hline $\begin{array}{c}\text { Powernse } \\
\text { (Operating Voltage) }\end{array}$ & $\begin{array}{c}\text { High } \\
\text { 0-100 Volts }\end{array}$ & $\begin{array}{c}\text { High } \\
\text { 0-60 Volts }\end{array}$ & $\begin{array}{c}\text { Low } \checkmark \\
0-2 \text { Volts }\end{array}$ \\
\hline $\begin{array}{c}\text { Image Quality } \\
\text { (in RMs WFE) }\end{array}$ & Fair & Low & High $\checkmark$ \\
Response Time & $50 \mathrm{~nm}$ & $200 \mathrm{~nm}$ & $20 \mathrm{~nm}$ \\
(in seconds) & Slow & Fast & Fast \\
Durability and & 20 & 0.1 & 0.1 \\
Robustness & Folymeric Liquid & Oil and Water & High $\checkmark$ \\
\hline
\end{tabular}




\section{Lemicense}

\subsection{Future Products}

After successful development of multi-focus lenses for camera phones,

LenSense will explore potential new applications including digital still cameras, video cameras, webcams, PDA's, medical imaging devices, microscopes, beam steering devices, interconnections, data reading in optical storage, and many other optical applications in which multiple focal lengths and compactness are desired 


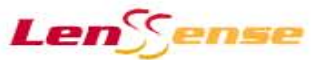

\subsection{Market Analysis Summary}

The target customer of LenSense is Nokia, the leading handset maker in the world. The mobile industry has been chosen by LenSense because:

$>$ LenSense technology has great competitive advantages and potential to improve the image quality of camera phones

$>$ Mobile market is one of the largest and fastest growing consumer electronics markets

$>$ LenSense suggests Nokia to develop an "Optics Research Center" at the heart of Optics Valley in Tucson, Arizona

There are more than 500 million camera phone users worldwide, who constitute the LenSense indirect target market. This figure explains the multi-billion dollar nature of the camera phone industry.

LenSense will concentrate on promoting viable technological solutions to prominent cell phone brands. Based on standards in the Rubber Manufacturers Association (RMA) manual, LenSense can be considered an engineering service provider that offers the design, fabrication, and testing.

Engineering services comprise establishments primarily engaged in applying physical laws and principles of engineering in design, development, and utilization of machines, materials, instruments, structures, processes, and systems. The assignments undertaken by these establishments may involve any of the following activities: provision of advice, preparation of feasibility studies. preparation of preliminary and final plan and designs, provision of technical services during the construction or installation phase, inspection and evaluation of engineering projects, and related services.

\subsection{Industry Trends}

According to The NPD Group, a leading consumer and retail information company, mobile phone sales in the U.S. reached 38 million units in the third quarter of 2007 alone. This represents an increase of $16 \%$ compared to the unit sales from the previous quarter.

NPD estimates $\$ 3.2$ billion sales in the third quarter 2007 , which shows a $47 \%$ increase compared to last year. The smart-phones sales during the third quarter increased from $4 \%$ of all phone sales in the third quarter of 2006 to $11 \%$ during 


\section{Lemicemse}

the same timeframe in 2007 - an increase of $163 \%$ year over year. A smartphone is a mobile phone offering advanced capabilities beyond a typical mobile phone, often with built-in camera.

Camera phones are important for their ability to capture spontaneous or unexpected moments. In addition, cell phone wireless communication features provide a great advantage over the stand-alone digital cameras. Apart from pleasure purposes, camera phones have found many new applications such as in business, journalism, and even law enforcement.

Most popular mobile phone features became more common in new phones sold. In the third quarter of $2007,72 \%$ of phones had Bluetooth capability and $90 \%$ of phones were equipped with built-in camera, which is a $34 \%$ increase since the third quarter of 2006 . A recent survey shows camera feature is the most used function of cell phones (Figure 6).

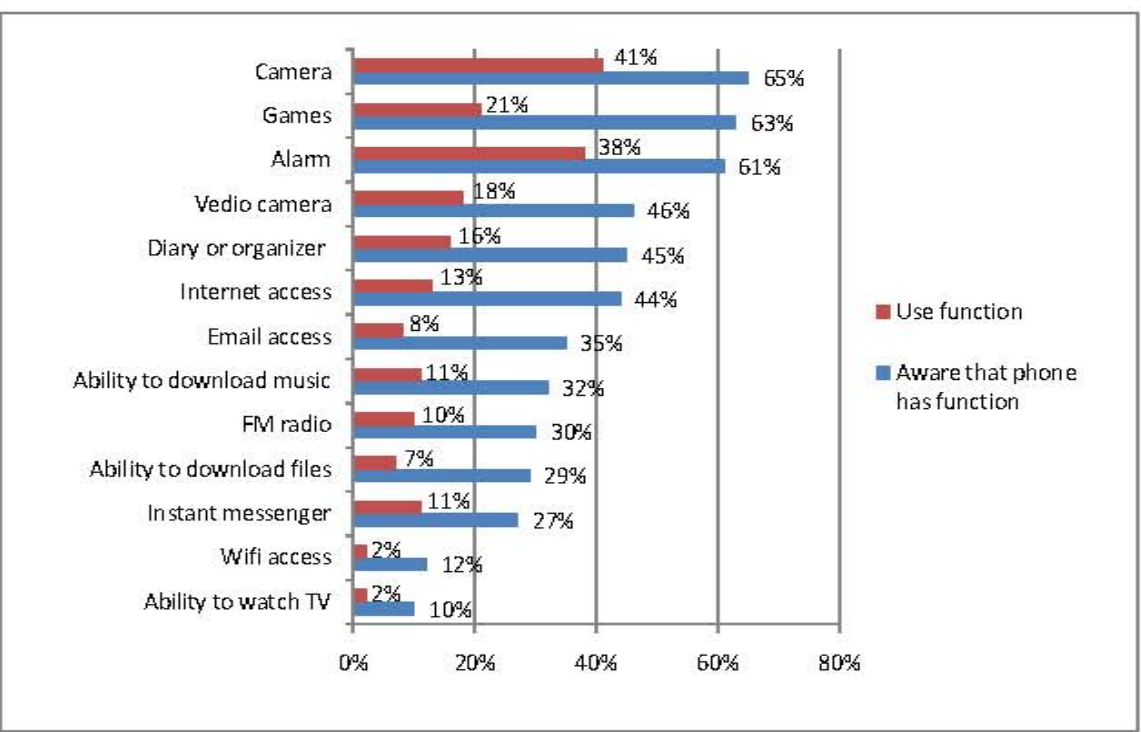

Figure 6: Percent of people who are aware of and use function [source: Ofcom research, July 2007.]

The mobile phone market is growing smarter. The nearly threefold increase in smart-phones shows that this once negligible niche is becoming a more influential force in the consumer market attracting entrants.

From 2003 to 2006, the unit sales of camera phone have been increasing rapidly at a growth rate of $15-20 \%$ annually. The explosive sales growth rates reflect the potential revenue to promote the company's new technology in cell phones. 

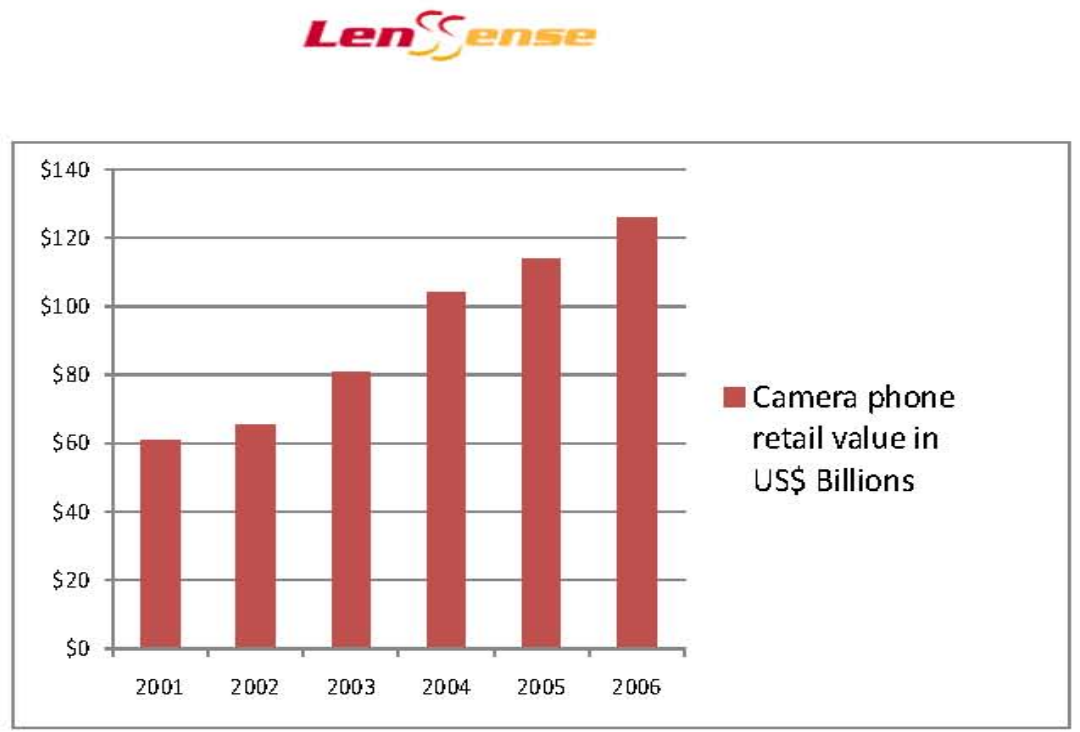

Figure 7: Camera phone sales in each year [source 2007 Wall Street Journal]

\subsection{Market Trends}

The direct customers of LenSense are Nokia as well as manufacturers of camera modules for Nokia. The indirect customers are more than 500 million the camera phone end-users worldwide. The number of camera phone users has been increasing rapidly since 2003 with the Asia Pacific being the largest growing region. Figure 3 shows the growing market trends in each region.

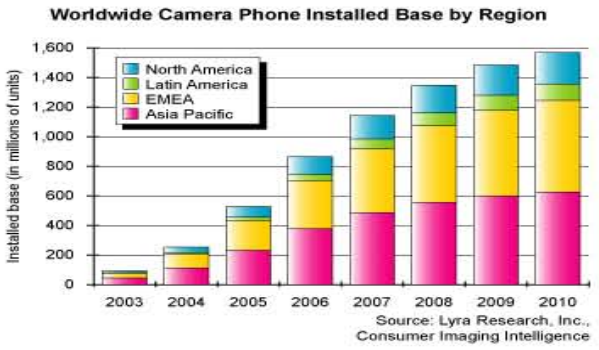

Figure 8: Worldwide camera phone installed base by region 


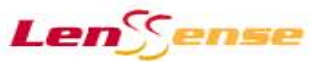

\subsection{Competitive Advantage for Nokia}

Nokia has the leading position in the cell phone industry. Currently, almost every cell phone has the camera function but almost none of them have the optical zoom function. With the LenSense technology. Nokia can be the first to introduce viable optical zoom and increase the leading edge with other competitors. The LenSense technology can also be used in several other applications including digital still cameras, camcorders, microscopes, medical devices such as endoscopes, PDA, and webcams that can open up new possibilities and additional revenues for Nokia.

In addition, Nokia can take advantage of cooperating with telecommunication companies such as AT\&T, T-mobile, and Verizon wireless. With LenSense's products, Nokia could provide cell phones with greater image quality which will attract more customers to join the data plan contracts. In this situation, the telecommunication companies will sign more contracts with Nokia to have a higher number of cell phones with better lenses.

\section{Potential New Markets}

\section{Digital Still Cameras}

Digital cameras have a trend to become smaller, less bulky, with better image quality. Digital camera market peaked very soon and will keep growing. In addition, shipments continued to be strong through 2006 to reach 94 million units. Average selling prices for cameras fell from $\$ 340$ (2004) to $\$ 295$ by the end of 2005 and $\$ 200$ by 2009 . ${ }^{4}$

\section{Camcorders (Video Camera)}

The digital camcorders provide tremendous levels of quality and a host of new, innovative features. Within those features, the image and video quality are the most important ones. According to manufacturers who track the camcorder sales through to consumers, the increase is actually more in the $13 \%$ to $14 \%$ range. That's still substantial when compared to growth rates in 2006 of $2 \%$ to $4 \%$ annually, giving manufacturers cause for optimism. ${ }^{5}$

${ }^{+}$CNET News.com

${ }^{5}$ EE Times 2007 


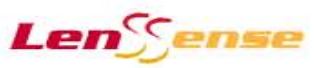

\section{PDA's}

In some developing markets, the handheld devices have been tremendously important in self-education, enabling users to continue learning outside the classroom once they have downloaded content through the PC. In this situation, video function is extremely important for PDAs which need better lenses. By 2008 IDC forecasts that the worldwide handheld market will ship 8.5 million, while converged device shipments will hit 130 million units. PDA users remain "fiercely loyal" to their handhelds. ${ }^{6}$

\section{Webcams}

With the advent of free Web-calling, instant messaging and high-quality imaging technology, video chat is fast gaining popularity among friends, family and co-workers. In this situation, image quality is important for webcam to shorten the distance between people. Webcam markets at $\$ 1.2$ billion in 2006 are anticipated to reach $\$ 6.2$ billion by 2013 . Webcam total market forecasts indicate that there is strong growth from a number of segments. ${ }^{7}$

\section{Medical Imaging Devices}

There are a lot of medical devices such as endoscopy, magnetic resonance imaging (MRI), microscopy, and positron emission tomography (PET) which would need great resolution of image while LenSense's optical zoom solution will be the potential solution for those medical devices.

${ }^{6}$ PDA market report by IDC 2007

'WinterGreen Research, Inc 


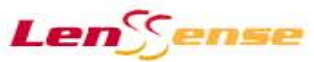

\subsection{Strategy and Implementation Summary}

LenSense business objectives will be achieved by combining sound marketing strategies and strong financial analysis with the innovative technology. The company will design and implement the prototype modules suitable for use in the camera phones. On the other hand, LenSense will document the manufacturing processes as well. LenSense will partner and build up a good relationship with the manufactures of camera modules for particular camera phone manufacturers. or sub-contract to independent semiconductor manufacturing companies for mass production. LenSense has a manufacturing integration plan that parallels development of its manufacturing capability with Nokia's new product design cycles by partnering with Nokia's manufacturing and design engineers now so as to sell LenSense product starting in Year 5.

\subsection{Strategy Pyramids}

The LenSense strategy is a two-phase process. The first phase includes research and design. During the first phase, LenSense will design and build prototypes, and document the manufacturing processes. The mission for the first phase is to integrate this new technology into camera phones. This will require financial budget to support the R\&D phase due to the equipment and material expenditure. LenSense looks forward to manufacturing design and integration meetings with Nokia over the next 5 years and having one of their engineers at our facility in Years 3-5.

The second phase is promotion. LenSense will build up close relationship with Nokia and manufactures of camera modules by focusing on the core value and differentiation from the existing solutions. During the second phase, the company will leverage relationships to generate sales. LenSense would gain the trust of the customers and recognition as a leader in the field by establishing market awareness and brand recognition.

\subsection{Value Proposition}

The LenSense value proposition is its high quality and expertise in cutting-edge technology which has the potential to revolutionize the entire zoom lens industry. LenSense design is simple and reliable, and our technology makes $100 \%$ testing a reality so Nokia does not receive defective product. LenSense will work with the six-sigma quality engineers and will not rush for lifecycle testing. The company will provide the prototype and feasibility analysis. 


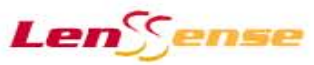

\subsection{Competitive Edge}

LenSense will differentiate itself by providing innovative technology that offers an advanced and affordable way to implement the new technology in camera phones. The company plans to enter the market early to gain a high market share of the zoom lens industry. As an innovative engineering service provider, LenSense will be attentive and flexible in meeting our customers' demands.

\subsection{Marketing Strategy}

LenSense promotes its innovative and quality lens solution that incorporates better features than competition (Table 1). Most importantly, our lenses operate at low voltages which means an increased battery life and longer talk time.

LenSense plans on building exclusive relationships with Nokia and if needed, to create an optics research center for Nokia in Tucson, Arizona that can also serve as the marketing "place" element or distribution.

\section{Market Mix}

\section{Product Strategy}

LenSense provides advanced lens solution which will target the mobile industry and then move into other applications. LenSense's intangible attributes are its partnership with Nokia, ability to meet or exceed customer expectations consistently, its speed in response to customers' demands, and its anticipation of new development. Those intangible attributes are difficult for competitors to copy, thereby giving LenSense a great competitive advantage.

\section{Price Strategy}

LenSense provides high-quality and progressive lenses which command a premium price. However, LenSense will partner with Nokia, which is the leading cell phone brand in the world and has an overall channel distribution. LenSense could reach a comparable low price by leveraging Nokia's supply chains and channels for mass production. By doing this, Nokia could also gain the large economic scales to invest the advanced lenses. 


\section{Lemeremse}

\section{Place Strategy}

LenSense seeks to develop a collaborative partnership with Nokia to produce the advanced lenses to be incorporated into Nokia products.

\section{Promotion Strategy}

LenSense will provide better technical support, maintenance, and testing for its quality lenses. At the same time, LenSense will keep developing this optical technology and keep the technology innovation.

\section{SWOT Analysis for LenSense}

\section{Strengths}

- Quality of lenses.

- Technology innovation.

- Location (Tucson, AZ, the heart of optics valley).

- Strong development team with a good mix of engineering and business

- Providing good supporting services including testing, maintenance. and technical supports

- Prototypes for potential markets such as digital cameras. camcorders, PDA, webcams, and medical device.

\section{Weakness}

- Lack of capital resources.

- Key members have less industrial experience.

- The development team lacks relationship with suppliers

- Single source of capital

\section{Opportunity}

- Mobile industry is growing rapidly

- Current cell phones lack optical zoom.

- Nokia is keen to develop new technology.

- The optical science research keeps improving at the world's best optical science program at the University of Arizona.

\section{Threats}

- Competitors such as Holochip and Varioptic.

- Theft of trade secret and other followers. 


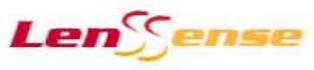

\subsection{Milestones}

The LenSense management team has established milestones to keep the business plan priorities in place. These milestones (Figure 9 ) will be updated monthly and milestones will be added as well. Once funded, a monthly meeting will be held to review and analyze the plan vs. actual work. Our shareholders and advisers will be provided with a copy of this monthly analysis and interact with LenSense management team on a bi-weekly basis.

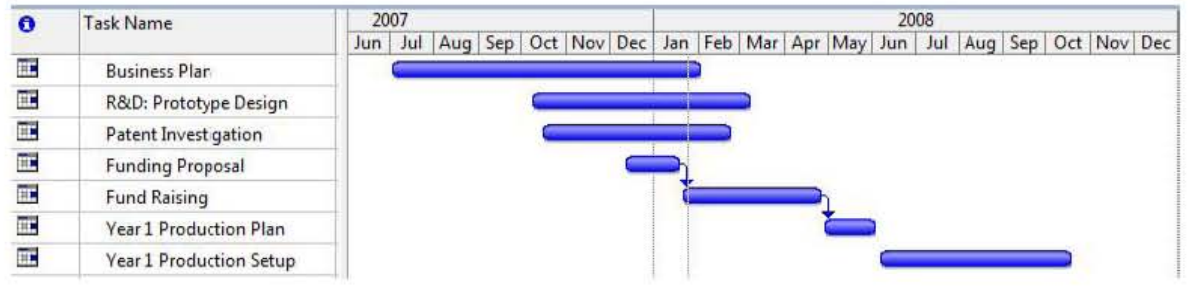

Figure 9: Pre-production Milestones 


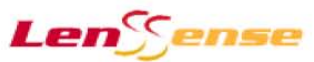

\subsection{Management Summary}

\subsection{Board of Managers}

\section{Pouria Valley (General/Product Manager):}

Mr. Valley is a PhD 2008 Optical Engineer at the College of Optical Sciences at the University of Arizona with a minor in Entrepreneurship from the Eller College of Management. He has strong engineering and leadership background. Mr. Valley has $5+$ years experience in the design, fabrication, and testing of electro-active liquid-crystal lenses He was the founding President of the International Society of Optical Engineering's (SPIE) student chapter in Arizona, and has won several awards including the SPIE scholarship, College of Optical Sciences' outstanding graduate student award, and University of Arizona's super achiever award.

\section{Jamie Huang (Marketing Manager):}

Ms. Huang is an MS in MIS 2008 at the Eller College of Management at the University of Arizona with a minor in entrepreneurship. Because of her strong leadership skills, she was elected by all the students in National Tsing-Hua University of Taiwan as the chairperson of the student council where she led 13 committees. Moreover, she interned as a system analyst at AHMC Healthcare Company in summer of 2007. She is the Financial Director at MIS Graduate Association. She is also the senior web developer at the Arizona Student Union, and hired by Deloitte Consulting LLP as a business technology analyst.

\section{Yan An (Financial Manager):}

Mr. An is the only selected candidate in the Accelerated MBA and MSMIS dual-degrees program 2008 at the Eller College of Management, University of Arizona. He has strong foundation in business management and IT, and has been playing multiple roles handling realworld projects for companies such as ON Semiconductor and Microsoft. $\mathrm{Mr}$. An is highly motivated, task driven, and endowed with integrity. $\mathrm{He}$ also has very diverse background and fluent in Japanese, Chinese, and English.
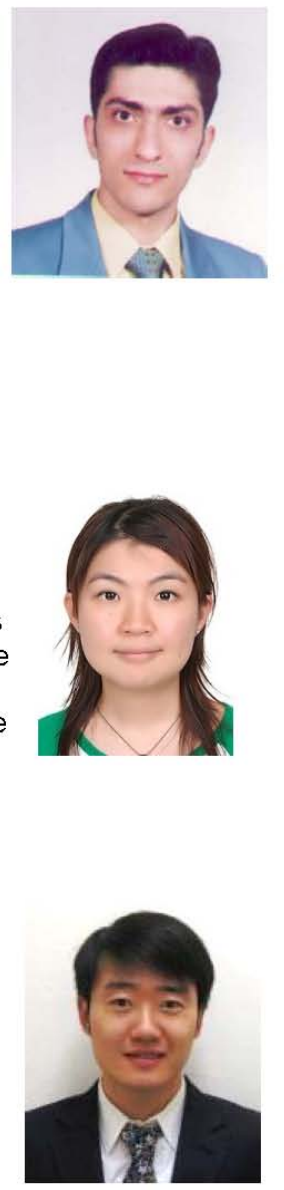


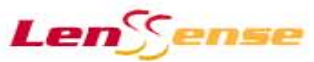

\subsection{Board of Advisors}

Dr. James C. Wyant

Professor Wyant is currently the Dean of College of Optical Sciences at the University of Arizona that is ranked as the best optics program in the United States by several prestigious organizations including the US News and World Report. Professor Wyant is the former President and co-founder of Wyko Corporation, and on the board of directors of Veeco, ILX Lightwave, Optics, and DMetrix. For his entrepreneurial activities, he has received several awards including the University of Arizona Entrepreneurial Fellow; Arizona's "Innovator of the Year" Product Award; the Tom Brown Excellence in Entrepreneurship Award; and the University of Arizona Technology Innovation Award.

$D$ Dr. Nasser Peyghambarian

Professor Peyghambarian is currently Chairman of the Board and founder of NPphotonics, Chair of Photonics and Lasers in the College of Optical Sciences, Professor of Optical Sciences, and Professor of Materials Science and Engineering at the University of Arizona. He also serves as Director of the Center for Optoelectronic Devices, Interconnect \& Packaging (COEDIP) and Director of the Photonics Initiative.

Mr. Jim Jindrick

Mr. Jindrick is the LenSense interim business development director with $30+$ years management experience encompassing strategic planning, business development, product research and engineering, and international marketing. $\mathrm{He}$ received seven U.S. utility patents and developed over 40 successful new products and high-tech enterprises that have generated over $\$ 750$ million lifetime revenue to date. Mr. Jindrick is a Mentor in Residence at the University of Arizona, Eller College of Management, McGuire

Entrepreneurship program which is consistently ranked as one of the best in the world by Entrepreneur. U.S. News and Worid Report, Fortune.

CNNMoney and Financial Times. 


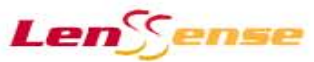

Mr. Michael J. Arnold

Mr. Arnold is the Director of the Engineering Management Program at the University of Arizona. He is the 1978 founder and former CEO for 23 years of Modular Mining Systems, a Tucson, Arizona based software and electronics manufacturer targeting the mining industry. The company grew from three principals, to over 300 employees including six foreign subsidiaries and placed number 187 on INC. Significant growth in exports earned the company the US Commerce Department's "E" Award in 1992. Komatsu, a large Japanese heawy equipment manufacturer, acquired the company in 1998 . He also served as Associate Director of the Entrepreneurship program in the Eller College of Management at UA prior to joining the Engineering College.

\section{Dr. Sharon Garrison}

Professor Garrison is currently Senior Lecturer at University of Arizona, former Professor of Finance and Chair of Finance and MBA Director at Florida Gulf Coast University. She has won several national grants and scholarships, and served on numerous academic committees and panels. She has also authored more than 150 publications, and her records of university and community services are exemplary. Professor Garrison is one of the financial advisors of LenSense.

\subsection{Operational Strategy}

LenSense will design and build the prototype for its camera phone lenses based on the state-of-the-art technology that is already demonstrated successfully for Ophthalmic applications. The company will further develop this technology for camera phone application, and secure intellectual properties through filing and/or licensing patents. Then, collaborative partnership contracts will be made with Nokia and a semiconductor manufacturer for the mass production of lenses.

In order to get the best performance for the operations, LenSense will focus primarily on the quality of products and services. In the first step, lenses are carefully engineered for mass manufacturability. Then, we will use automated assembly, quality control machinery, lean processes, and six-sigma reliability tests from the time the product arrives at the manufacturing plant to the end of the warranty period. LenSense will follow the Standard Mobile Imaging Architecture (SMIA) standard which is the cornerstone of Nokia's high-volume camera strategy. 


\section{Lemicense}

\subsection{Financial Plan}

The goal of LenSense is to supply a minimum of $25 \%$ of camera phone lenses shipped by Nokia annually. With expected annual growth rate between $15-20 \%$, sales projection for Year 1 is at $\$ 60$ million, and increasing to $\$ 124$ million in Year 5 with EBITDA exceeding $\$ 40$ million. Assumptions made in deriving this financial plan are listed in the appendix, and all the estimates are obtained by taking into account of the economic fluctuations of high, medium, and low demands.

It is certain that LenSense prefers to partner with Nokia in designing and manufacturing lenses for its camera phone modules. The attractiveness of Nokia is its famous low-cost mass production and well-established global supply chain. Nevertheless, in order to project critical financial figures, the team now assumes that LenSense is a fully self-contained enterprise, with its own supply chain and contracted manufacturing resources.

\subsection{Demand Projection}

LenSense conservatively estimates to supply 100 million lenses to Nokia in the $5^{\text {th }}$ year of operation. It is achieved based on an annual growth rate between $15-$ $20 \%$ by benchmarking Nokia, Motorola, and Samsung. The rationales behind this estimation are discussed below.

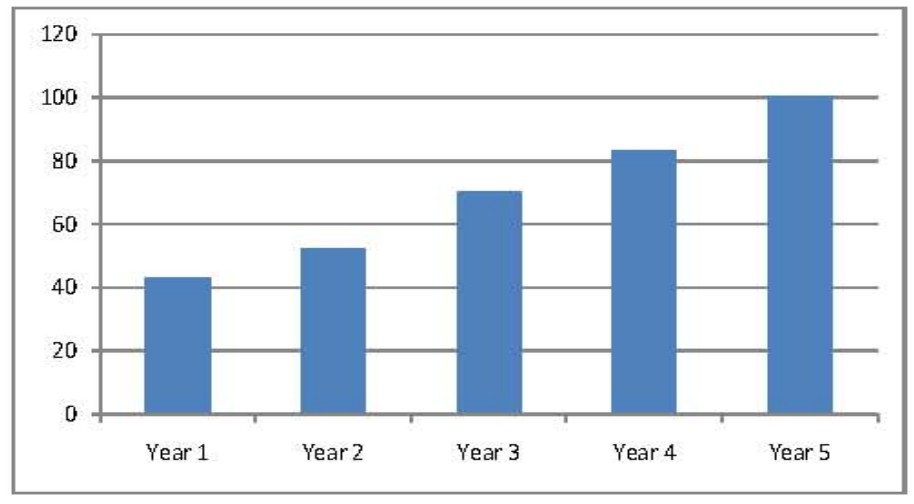

Figure 10: Projected Sales Quantity (in M's) 


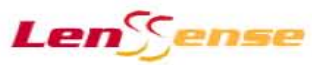

According to a recent report from iSuppli Corporation, a leading marketing research firm in the electronics value chain, 285 million phones were shipped by handset makers in the third quarter of 2007. As Nokia continued to dominate the handset market, its market share climbed from $38.9 \%$ in 2006 to $39.2 \%$ in 2007 . Figure 11 shows the major players in this industry and their corresponding market share percentages.

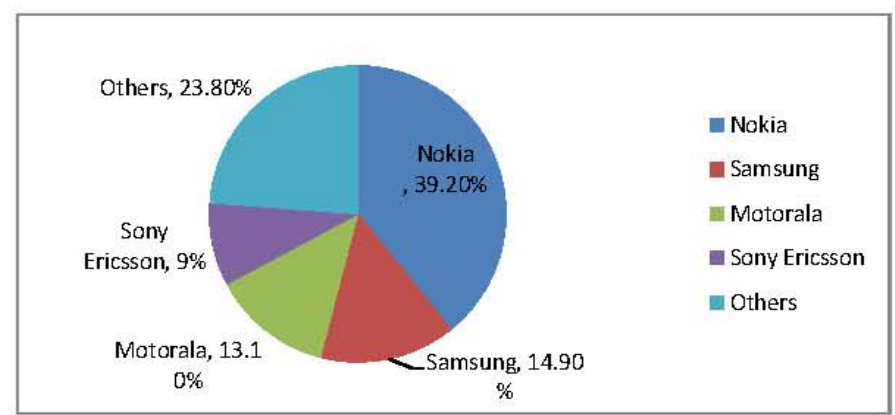

Figure 11: Market Share [source: iSuppli]

Nokia shipped approximately 110 million handsets per quarter and a total of 450 million units in 2007 . In addition, based on a research conducted by InfoTrends, camera phones are expected to account for $89 \%$ of all mobile phone han dsets shipped by 2009 . That is, out of Nokia's 450 million handsets, 410 million would be camera phones in 2009 . Without taking into account Nokia's $15-20 \%$ annual growth rate, LenSense is confident to expect a demand of 100 million units from Nokia in the $5^{\text {th }}$ year of operation, less than a quarter of Nokia's total handsets production in 2007.

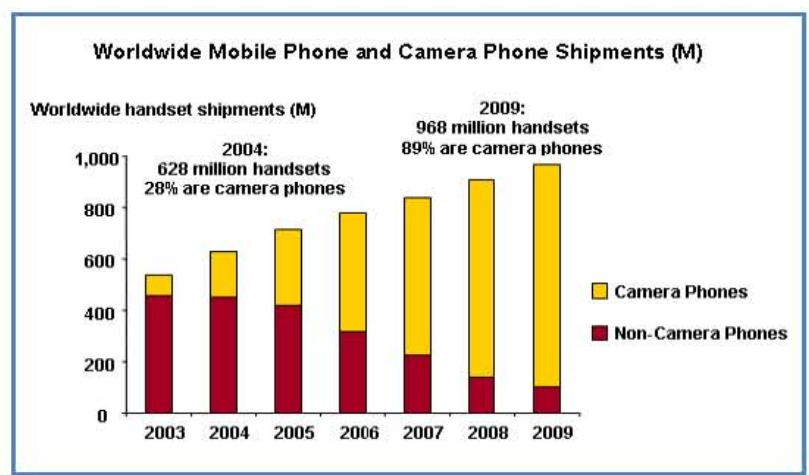

Figure 12 [source: InfoTrends] 


\section{Lemecense}

\subsection{Projected Profit}

The following income statement is created by leveraging facts such as economic fluctuations and emergence of competitors. In addition, the unit cost is configured to decrease by $10 \%$ as the quantity increases by $15 \%$. In Year 1 of operation. LenSense projects a net profit of $\$ 14$ million. The amount is expected to be doubled in 5 years as the technology reaches maturity and demand increases. The operation starts from the $2^{\text {nd }}$ months

\begin{tabular}{|c|c|c|c|c|c|}
\hline Projected Income Statements (in K's) & Year 1 & Year 2 & Year 3 & Year 4 & Year 5 \\
\hline SALES & $\$ 60,000$ & $\$ 72,450$ & $\$ 87,450$ & $\$ 103,500$ & $\$ 124,500$ \\
\hline \multicolumn{6}{|l|}{ COST OF SALES } \\
\hline Materials & $\$ 33,572$ & $\$ 40,572$ & $\$ 48,972$ & $\$ 57,960$ & $\$ 69,720$ \\
\hline Labor & $\$ 1,850$ & $\$ 2,079$ & $\$ 3,921$ & $\$ 3,921$ & $\$ 6,091$ \\
\hline Taxes and Benefits & $\$ 272$ & $\$ 302$ & $\$ 680$ & $\$ 680$ & $\$ 925$ \\
\hline TOTAL COST OF SALES & $\$ 35,694$ & $\$ 42,953$ & $\$ 53,573$ & $\$ 62,561$ & $\$ 76,736$ \\
\hline GROSS MARGIN & $\$ 24,306$ & $\$ 29,497$ & $\$ 33,877$ & $\$ 40,939$ & $\$ 47.764$ \\
\hline \multicolumn{6}{|l|}{ OPERATING EXPENSES } \\
\hline Salaries and wages & $\$ 1,500$ & $\$ 1,700$ & $\$ 1,900$ & $\$ 2,200$ & $\$ 2,500$ \\
\hline Payroll taxes & $\$ 124$ & $\$ 150$ & $\$ 185$ & $\$ 200$ & $\$ 230$ \\
\hline Employee benefits & $\$ 47$ & $\$ 55$ & $\$ 75$ & $\$ 95$ & $\$ 115$ \\
\hline Depreciation & $\$ 188$ & $\$ 205$ & $\$ 205$ & $\$ 205$ & $\$ 205$ \\
\hline Advertising & $\$ 25$ & $\$ 35$ & $\$ 40$ & $\$ 45$ & $\$ 48$ \\
\hline Insurance & $\$ 46$ & $\$ 50$ & $\$ 65$ & $\$ 75$ & $\$ 85$ \\
\hline $\mathrm{R} \& \mathrm{D}$ & $\$ 825$ & $\$ 900$ & $\$ 1,000$ & $\$ 1,200$ & $\$ 1,350$ \\
\hline IT & $\$ 73$ & $\$ 80$ & $\$ 85$ & $\$ 90$ & $\$ 100$ \\
\hline Fabrication F ac ility Rental & $\$ 917$ & $\$ 1,000$ & $\$ 1,200$ & $\$ 1.500$ & $\$ 1,700$ \\
\hline TOTAL OPERATING EXPENSES & $\$ 3,745$ & $\$ 4,175$ & $\$ 4.755$ & $\$ 5,610$ & $\$ 6,333$ \\
\hline \multicolumn{6}{|l|}{ OPERATING PROFIT (LOSS) } \\
\hline BEF ORE INTEREST AND TAXES & $\$ 20,561$ & $\$ 25,322$ & $\$ 29,122$ & $\$ 35,329$ & $\$ 41.431$ \\
\hline INTEREST EXPENSE & $\$ 0$ & $\$ 0$ & $\$ 0$ & $\$ 0$ & $\$ 0$ \\
\hline PROFIT (LOSS) BEFORE TAXES & $\$ 20,561$ & $\$ 25,322$ & $\$ 29,122$ & $\$ 35,329$ & $\$ 41,431$ \\
\hline INCOME TAXES & $-\$ 6,072$ & $-\$ 7,583$ & $-\$ 8,893$ & $-\$ 11,021$ & $-\$ 13,069$ \\
\hline NET PROFIT (LOSS) & $\$ 14,489$ & $\$ 17,739$ & $\$ 20,229$ & $\$ 24,308$ & $\$ 28,362$ \\
\hline EBITDA & $\$ 20,749$ & $\$ 25,527$ & $\$ 29,327$ & $\$ 35,534$ & $\$ 41,636$ \\
\hline
\end{tabular}




\section{Lemicemse}

\subsection{Projected Cash Flow}

The projected annual cash flow is shown in the table below. Thank to the low cost mass production, LenSense is expected to see a positive cash flow of $\$ 17$ million in the first year, and increasing to $\$ 111$ million in just 5 years. It is necessary to inject these excess cash on hand into R\&D and product line expansion on yearly basis.

\begin{tabular}{|c|c|c|c|c|c|}
\hline Projected Cash Flows (in K's) & Year 1 & Year 2 & Year 3 & Year 4 & Year 5 \\
\hline \multicolumn{6}{|l|}{ CASH FLOWS FROM OPERATIONS } \\
\hline Net inc ome & $\$ 14,168$ & $\$ 17,694$ & $\$ 20,751$ & $\$ 25,717$ & $\$ 30,494$ \\
\hline \multicolumn{6}{|l|}{ Adjustments to reconcile net income } \\
\hline Depreciation & $\$ 187$ & $\$ 205$ & $\$ 205$ & $\$ 205$ & $\$ 205$ \\
\hline Accounts recewable & $-\$ 10,900$ & $-\$ 1,175$ & $-\$ 2,500$ & $-\$ 2,675$ & $-\$ 3,500$ \\
\hline Inventory & $\$ 0$ & $\$ 0$ & $\$ 0$ & $\$ 0$ & $\$ 0$ \\
\hline Accounts payable & $\$ 6,104$ & $\$ 658$ & $\$ 1,400$ & $\$ 1,498$ & $\$ 1,960$ \\
\hline Other current payables & $\$ 0$ & $\$ 0$ & $\$ 0$ & $\$ 0$ & $\$ 0$ \\
\hline \multicolumn{6}{|l|}{ TOTAL CASHFLOWS FROM } \\
\hline OPERATIONS & $\$ 9,560$ & $\$ 17,382$ & $\$ 19,856$ & $\$ 24,745$ & $\$ 29,159$ \\
\hline \multicolumn{6}{|l|}{ CASH FLOWS FROM INVESTING } \\
\hline ACT VITIES & & & & & \\
\hline Purchase of equipment & $-\$ 1,470$ & $\$ 0$ & $\$ 0$ & $\$ 0$ & $\$ 0$ \\
\hline \multicolumn{6}{|l|}{ TOTAL CASH FLOWS FROM } \\
\hline INVESTING ACTIVITES & $-\$ 1,470$ & $\$ 0$ & $\$ 0$ & $\$ 0$ & $\$ 0$ \\
\hline \multicolumn{6}{|l|}{ CASH FLOW BEF ORE } \\
\hline FINANCING & $\$ 8,090$ & $\$ 17,382$ & $\$ 19,856$ & $\$ 24,745$ & $\$ 29,159$ \\
\hline \multicolumn{6}{|l|}{ CASH FLOWS FROM FINANCING } \\
\hline \multicolumn{6}{|l|}{ ACTVITIES } \\
\hline \multicolumn{6}{|l|}{ CASH FLOW BEF ORE } \\
\hline EQUITY FINANCING & $\$ 8,090$ & $\$ 17,382$ & $\$ 19,856$ & $\$ 24,745$ & $\$ 29,159$ \\
\hline Sales of stock & $\$ 9,590$ & $\$ 0$ & $\$ 0$ & $\$ 0$ & $\$ 0$ \\
\hline Repurchase of stock & $\$ 0$ & $\$ 0$ & $\$ 0$ & $\$ 0$ & $\$ 0$ \\
\hline \multicolumn{6}{|l|}{ TOTAL CASH FLOWS FROM } \\
\hline FINANCING ACTIVITIES & $\$ 9,590$ & $\$ 0$ & $\$ 0$ & $\$ 0$ & $\$ 0$ \\
\hline NET CASH FLOWS & $\$ 17,680$ & $\$ 17,382$ & $\$ 19,856$ & $\$ 24,745$ & $\$ 29,159$ \\
\hline CASH, BEGINNING OF PERIOD & $\$ 0$ & $\$ 17,680$ & $\$ 35,063$ & $\$ 54,919$ & $\$ 79,664$ \\
\hline CASH, END OF PERIOD & $\$ 17,700$ & $\$ 35,000$ & $\$ 55,000$ & $\$ 80,000$ & $\$ 109,000$ \\
\hline
\end{tabular}




\subsection{Projected Balance Sheet}

The projected balance sheet also indicates that cash constitutes a significant weight of the assets of LenSense. Because "Cash is the King!" the team should utilize the capital in reinvesting itself and configure the numbers into financial statements.

\begin{tabular}{|c|c|c|c|c|c|}
\hline Projected Balance Sheets (in K's) & Year 1 & Year 2 & Year 3 & Year 4 & Year 5 \\
\hline \multicolumn{6}{|l|}{ ASSETS } \\
\hline \multicolumn{6}{|l|}{ Current Assets } \\
\hline Cash & $\$ 17,680$ & $\$ 35,063$ & $\$ 54,919$ & $\$ 79,664$ & $\$ 108,823$ \\
\hline Accounts Receivable & $\$ 10,900$ & $\$ 12,075$ & $\$ 14,575$ & $\$ 17,250$ & $\$ 20,750$ \\
\hline Inventory & $\$ 0$ & $\$ 0$ & $\$ 0$ & $\$ 0$ & $\$ 0$ \\
\hline Other & $\$ 0$ & $\$ 0$ & $\$ 0$ & $\$ 0$ & $\$ 0$ \\
\hline Total Current Assets & $\$ 28,580$ & $\$ 47,138$ & $\$ 69,494$ & $\$ 96,914$ & $\$ 129,573$ \\
\hline Property and Equipment & $\$ 1,470$ & $\$ 1,470$ & $\$ 1,470$ & $\$ 1,470$ & $\$ 1,470$ \\
\hline (less accumulated depreciation) & $-\$ 188$ & $-\$ 393$ & $-\$ 598$ & $-\$ 803$ & $-\$ 1,001$ \\
\hline Net Property and Equipment & $\$ 1,282$ & $\$ 1,077$ & $\$ 872$ & $\$ 667$ & $\$ 462$ \\
\hline Other Assets & $\$ 0$ & $\$ 0$ & $\$ 0$ & $\$ 0$ & $\$ 0$ \\
\hline TOTALASSETS & $\$ 29,862$ & $\$ 48,215$ & $\$ 70,366$ & $\$ 97,581$ & $\$ 130,035$ \\
\hline \multicolumn{6}{|l|}{$\begin{array}{l}\text { UABIUTIES AND STOCKHOLDERS' EQUTY } \\
\text { Liabilities }\end{array}$} \\
\hline \multicolumn{6}{|l|}{ Current Liabilities } \\
\hline Accounts Payable & $\$ 6,104$ & $\$ 6,762$ & $\$ 8,162$ & $\$ 9,660$ & $\$ 11,620$ \\
\hline $\begin{array}{l}\text { Other Current } \\
\text { Payables }\end{array}$ & $\$ 0$ & $\$ 0$ & $\$ 0$ & $\$ 0$ & $\$ 0$ \\
\hline Total Current Liabilities & $\$ 6,104$ & $\$ 6,762$ & $\$ 8,162$ & $\$ 9,660$ & $\$ 11,620$ \\
\hline Long-Term Debt & $\$ 0$ & $\$ 0$ & $\$ 0$ & $\$ 0$ & $\$ 0$ \\
\hline Total Liabilities & $\$ 6,104$ & $\$ 6,762$ & $\$ 8,162$ & $\$ 9,660$ & $\$ 1,162$ \\
\hline \multicolumn{6}{|l|}{ Stockholders' Equity } \\
\hline Paid-in Capital & $\$ 9,590$ & $\$ 9,590$ & $\$ 9,590$ & $\$ 9,590$ & $\$ 9,590$ \\
\hline Retained Earnings & $\$ 14,168$ & $\$ 31,863$ & $\$ 52,614$ & $\$ 78,331$ & $\$ 108,825$ \\
\hline Total Stockholders' Equity & $\$ 23,758$ & $\$ 41,453$ & $\$ 62,204$ & $\$ 87,921$ & $\$ 118,415$ \\
\hline TOTAL பABIUTIES AND STOCKHOLDERS' EQUITY & $\$ 29,862$ & $\$ 48,215$ & $\$ 70,366$ & $\$ 97,581$ & $\$ 130,035$ \\
\hline
\end{tabular}




\section{Lemicemse}

\subsection{Suggested Vemure's Valuation}

LenSense is able to utilize industrial ratio in estimating the value of the venture. Nokia, Motorola, and Shamir Optical Industry are benchmarked in this case. As shown in the table 3 , the industrial ratio of these companies ranges between 9 and 18. As these companies are major handset or lens makers, LenSense is likely to have similar growth patterns. Therefore, the company can average their ratios and adopt 13.2 as the firm's Industry Ratio. Given the projected EBITDA in year 5 (approximately $\$ 41$ million), the terminal value of LenSense is projected to reach $\$ 600$ million at the end of Year 5 .

Table 3: Industrial Ratios [source: Yahoo Finance]

\begin{tabular}{|l|c|c|c|}
\hline & $\begin{array}{l}\text { NOKIA } \\
\text { (in B's) }\end{array}$ & $\begin{array}{l}\text { MOTOROLA } \\
\text { (in M's) }\end{array}$ & $\begin{array}{l}\text { Shamir Optical } \\
\text { Industry (in M's) }\end{array}$ \\
\hline Market Cap & 145 & 35 & 141 \\
\hline EBITDA & 12 & 2 & 16 \\
\hline Ratio & 12 & 18 & 9 \\
\hline
\end{tabular}

Information of Break-even analysis, NPV of Cash flow, and Return to Investors are available upon request. 


\section{Lemecemse}

\section{Appendix}

\section{Appendix 1: Assumptions to Financial Statemems:}

- Tax: The company starts in the form of LLC with federal tax rate of $25 \%$ and a state tax of $5 \%$.

- Cost of Capital: $16.5 \%$

- Terminal Year: 5

- Purchases \& Depreciation: No production in the $1^{\text {st }}$ month. During this month, fabrication equipments and licensing agreement will be purchased

- Fabrication equipments: $\$ 800,000$ (Depreciation: 6 year 0 value)

- Licensing agreement: $\$ 600,000$ (Expire in 10 years)

- Projected Selling Quantity (Year 1-5):

- Year 1 (11 months): 48 million units

- Year 2: 58 million units

- Year 3: 70 million units

- Year 4: 83 million units

- Year 5: 100 million units

- Operating Expenses: Insurance, advertising, R\&D, IT, and fabrication facility rental costs are configured to increase as sales increases

- Economic Fluctuations:

- Medium demand is $30 \%$ reduction from high demand

- Low demand is $50 \%$ reduction from high demand

- $50 \%$ chance for high demand, $30 \%$ for medium, and $20 \%$ for low demand

- Collection \& Payment Options: The collection cycle and payment cycle options are 60 days. The purchase lead time is just-in-time. No Bad Debts is expected (corporation guaranteed)

(More information is available upon request) 


\section{Lenctemse}

\section{Appendix 2: Proceedings of the National Academy of Sciences' Article}

\section{Switchable electro-optic diffractive lens with high efficiency for ophthalmic applications}

Guoqiang Li*t, David L. Mathine*, Pouria Valley*, Pekka Āyrās*, Joshua N. Haddock* , M. S. Giridhar*, Gregory Williby** Jim Schwiegerling*5, Gerald R. Meredith", Bernard Kippelen", Seppo Honkanen*, and Nasser Peyghambarian*"

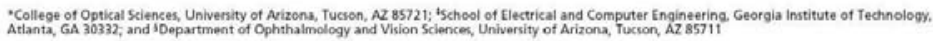

Communicated by Nicolass Dloembergen, University of Arizona, Tucion, AZ, February 2, 2006 (received for review December 10, 2005)

Presbyopia is an age-related loss of accommodation of the human retardation from the refractive lens, resulting in multiple Fresnel near objects. Assuming as inability to shift focus from distant to zones. The phase jump at each zone boundary is $2 \pi$ for the desi near objects. Assuming no refractive error, presbyopes have clear near objects. Area-divided bifocal lenses are one example of a treatment for this problem. However, the field of view is limited in such eyeglasses, requiring the user to gaze down to accomplish fort Here, we report on previously undescribed wist ascom. liquid-crystal diffractive lenses that can adaptively change their focusing power. The opera tion of these spectacle lenses is based electrical control of the refractive index of a 5 - $\mu \mathrm{m}$-thick layer of nematic liquid crystal using a circular array of photolithographi. cally defined transparent electrodes. It operates with high transmission, low voitage $(<2 \mathrm{~V}$ mm), fast response $(<1$ sec), diffraction eficiency $50 \%$, small abenation, and a power-failure-sate of -the-art liquid-crystal diffractive lenses for visson care and o ther applications. They have the potential of revolutionizing the field of presbyopia correction when combined with automatic adjustable focusing power.

ophthalmic lens | switchable lers | vision correction

The use of nematic liquid crystals to implement switchable lenses has been proposed previously but had limited success lenses incorporating comvex and concrve substrates have been demonstrated $(2,3)$. However, the large thickness of the liquid crystal layers $(>400 \mu \mathrm{m})$ make their response and recovery times long and their transmission low beeause of optical seattering. To reduce the thickness of the active layer, surface relief Fresne lens substrates tasc been proposed (4). However, in this geomis not desirable for oplthelnie applientions where a bes of is not desirable for ophatialmic applications where a loss of during a critical distance vision task such as driving. In other approaches, thin uniform layen of liquid crystal were used, and refractive lenses were produced by the use of discrete electrodes (3), continuous highly resistive electrodes (6), or spatially dis. tributed electric fields (microlenses) (7). However, in these lenses cither the range or focal length or hicir smail diameter a photolithographically patterned thin diffractive lens with large aperture, fast response time, ration to overcome these limitations. Although high-efficiency liquid-crystal-based diffractive devices have been demonstrated for beam-stecring $(8,9)$, less effort was given to the development

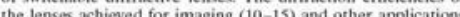
the lenses achicved for imaging (10-15) and other applications applications.

Fig. 1a compares the shape (phase profile) of a refractive lens (dashed line) with an ideal diffractive lens (dotted line). The diffractive lens is produced by removing the multiple $2 \pi$-phas

$$
r_{m}=\sqrt{2 m \Lambda,}, \quad m=1,2, \ldots, M .
$$

where $m$ is a counting index that refers to successive Fresnel zone tarting in the center, $\lambda$ is the wavelength, and $f$ is the focal ength. To digitize the process, the continuous phase profile in each zonc is divided into multiple subzones with a series of discrete phase levels (19) ("staircase" st
outer radius of each subzone is given by

$$
r_{m, n}=\sqrt{2[(m-1)+n / L] \mathrm{N} f}, \quad n=1,2, \ldots, L, \quad[1 \mathrm{~b}]
$$

where $L$ is the number of phase levels per zone, and $n$ is the
counting index of the individual phase levels. Diffraction efficounting index of the indiswa pumber of subzones $L$, reachciency increases by increasing the number
ing maximum values of $40.5 \%, 81.1 \%$, and $95.0 \%$ for lenses with two, four, and eight phase levels per zone, respectively. Diffractive lenses with eight subzones, $10-\mathrm{mm}$ diameters, and respectively) were demonstrated at the peak of the human photopic response, $555 \mathrm{~nm}$. The schematic drawings of the electrode pattern and the fabrication procedure are shown in Fig. $1 b$ and $c$, respectively. Using photolithographic techniques, concentric and rotationally symmetric transparent indium tin axide electrodes (S0 nim in hickness), whose radii were deter-

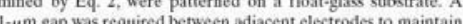
electrical isolation and ensure a smooth transition of the phase profile introduced by the liquid erystal. Over the patterned indium tin oxide, a 200 -nm-thick electrically insulating layer of $\mathrm{SiO}_{2}$ is sputtered and into which small via openings $(3 \times 3 \mu \mathrm{m})$ were etched, allowing electrical contact to be made to the andertying electrodes. An electrically conductive layer of Al is subsequently sputtered over the insulating layer to fill the vias pendent electrical bus bars ( 6 - $\mu$ m wide within the lens). Each bus bar connects the discrete phase level electrodes of equal count. ing index $n$ in all Fresnel zones (as shown in Fig. 1b) such that only eight external electrical connections (plus one ground connection) are required per lens.

The patterned substrate, as well as an additional substrate with round, were spin costed with poly(vinyl aleohol) to arical liquid-crystal alignment layer. The alignment layers were rubhed with a velvet cloth to achieve homogeneous alignment, and the two substrates were assembled. The commercial nematic liquid 
Lemicemse
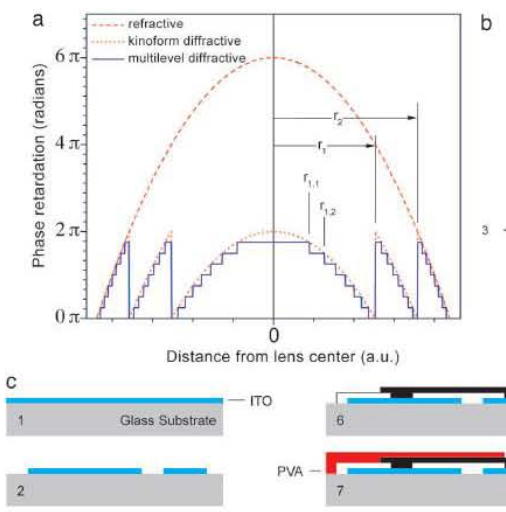

PVA
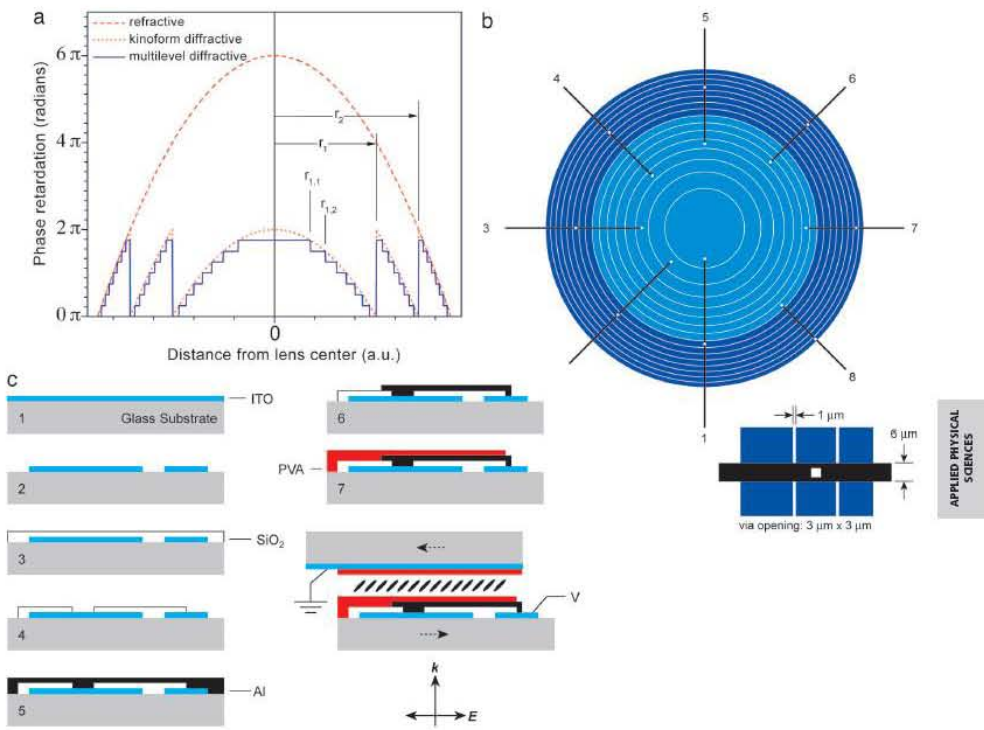

Fig. 1. Adaptive liquid-crystal diffractive lens. (a) Dashed line, phase profile of a conventional refractive lens, dotted line, phase proffle to achieve a difftractive
lenss staircase structure, multilievel quantization approximates the continuous quadratic blaze profile. a.u, arbitrary units. (b) Layout of the one-layer electrode pattern (two central zones shown). Adjacent zones are distinguished by color. An electrical insulation layer with vias i s sadded (viasshown with white dots). Each (c) Processing stepsfor fabrication of the patterned electrodes and the conductive lines. The structure of the liquid-crystal lens is shown in the lower right, where $k$ is the wave vector, and $E$ is the polarization state of the incident light.

crystal E7 (Merck) was used as the electro-optic medium and was filled by capillary action into the empty cell at a temperature above the clearing point $\left(60^{\circ} \mathrm{C}\right)$ and then cooled at $1{ }^{\circ} \mathrm{C} / \mathrm{min}$ to connected to the drive electronics. The drive electronics consis pendently controlled output channels. Each channel generates a modified square waveform with variable peak-to-peak amplitude between 0 and $5 \mathrm{~V}$. operated properly and provided discrete phase changes (Fig. $2 a$ ).
Eight optimized drive voltages with amplitudes between 0 and 2 rms produced a maximum first-order diffraction efficiency or $1 \%$, near the $95 \%$ predicted by scalar diffraction theory. The measured diffraction efficiency as a function of lens area (see

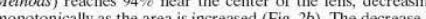
due to the fact that phase distortion caused by the fringing field the zone boundaries has more zones as the width of each electrode becomes smaller. At the

Results edges of the electrodes, the electric field lines are not perpen-

The lens showed excellent performance (because of space limitations, we only describe heresults for the 1-diopter lens). spectrum, a value that can be increased by the use of ophthaln quality substrates and antireflection coatings. Monochromatic (543.5 nim) polarized hicroscopy inages of the lens under cause the phase transitions at she zar be not as reducing the diffraction efficiencies $(20,21)$. The focused spot size is $\approx 135 \mu \mathrm{m}$, which is also close to the diffraction-limit value $133 \mu \mathrm{m}$. The lens shows subsecond switching time Interferometric measurements af $543.5 \mathrm{~nm}$ (see Methods) 
Lemceramse

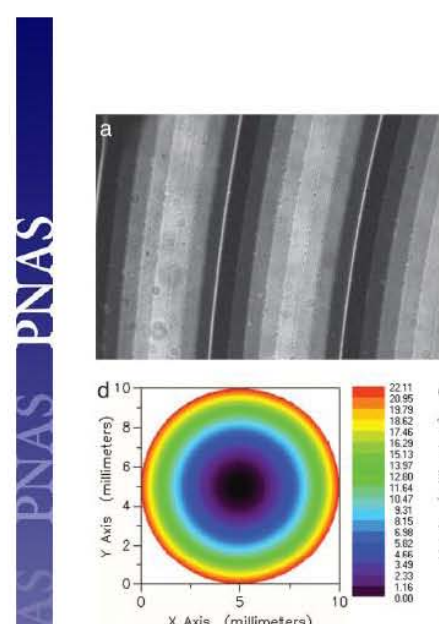

$x$ Axis (millimeters)
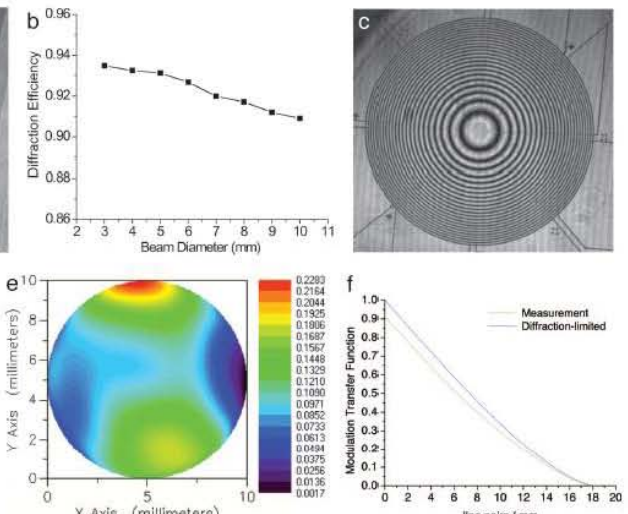

Fig. 2. Characterization of the 1-diopter lens. (a) Electro-optic response of the lens obtained with polarized microscope. (b) Diffraction efficiency as a function

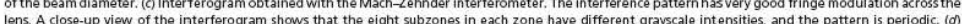
Unwrapped phase map for a 10-mm aperture. (e) Phase map of the unwrapped phase minus tititing and focusing. (f) MTF of the lens. The green line is obtained from the measurement data, and the blue line is for a diffraction-limited lens. The value at low spatial frequency is determined by the diffraction efficiency of

of the optical power is observed in interferogram of the lens in the optically active state (Fig. 2c). The unwrapped phase map of the lens is shown in Fig. $2 d$ with a peak-to-valley optical path
length of $23.05 \lambda$. The focusing power was estimated to be 1.002
diopter, in excellent agreement with the design value. Very good diopter, in excellent agreener indicating small aberrations. Higher-order aberrations were estimated by analyzing the difference between the measured wavefront and a best-fit spherical wave and tilt (Fig. 2e). The
peak-to-valley range of the difference is $0.241 \lambda$, and the rms value is $0.039 \lambda$, which is comparable with a high-quality reading glass. The modulation transter function (Mill) indicated near lens, as shown in Fie 2, make the switchable lens surable for ophthalmic applications.

To test the imaging properties of the lens, a model human eye was constructed by using a fixed, +60 diopter achromatic doublet glass lens and a monochrome charge-coupled device
(CCD) with a filter to match the human photopic response. (CCD) with a filter to match the human photopic response.
Because homogeneously aligned nematic liquid crystals are polarization sensitive, two lenses with orthogonal alignment directions were used in series to create a single polarizationinsensitive lens. Two such lenses were aligned and cemented together. To simulate a typical near-vision task such as reading,
a double-element lens was placed in front of the model eye and used to image a test object illuminated with unpolarized white light placed $30 \mathrm{~cm}$ in front of the lens. As can be seen in Fig. $3 a$, by switching on the diffractive lens the image is brough into focus $(\mathrm{Fig} .3 \mathrm{~b}$ ). The double-element lens has excellent optica transmission. To test the imaging performance of the lenses with actual human subjects, a pair of test spectacles has been con-

structed (Fig. 4), and initial clinical results agree well with the model eye test. When the electro-optic lenses are both in the inactive state, there is no netictable degradation in the quality of the distant vision. For chromatic abertation, an achromatic diffractive lens can be designed by introducing $p 2 \pi(p>1$, integer) phase jun at the aberration brain is adapted to a certain degree of chromatic aberration, balancing the dispersion of the diffractive lens and the eye is less desirable. More clinical study needs to be performed on this aspect.

\section{Conclusion}

In conclusion, we have demonstrated switchable liquid-crystal diffractive lenses with high diffraction efficiency, high optical mance. These flat lenses are highly promising to replace conventional area division refractive, multifocal spectacle lenses used by presbyopes. They have the potential of revolutionizing the field of presbyopia correction when it is combined with automatic adjustable focusing power. Negative focusing powers also can be achieved with the same lenses by changing the sign of the slope of the applied voltages. The use of these lenses is not limited to ophthalmology but can be extended to numerous other

Methods

When the device is fabricated, various optical characterizations are performed.

$6102 \mid$ www.pnas.org/cql/dol/10.1073/pnas 0600550103 


\section{Leméneme}

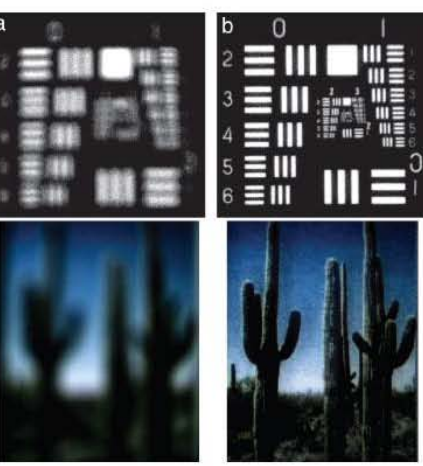

Fig. 3. Hybrid imaging using the 1-diopter electroactive diffractive lenswith correction to the model eye. (a) The object is pplaced at a a reading distance ( $\sim 30$ cm). The image is severely out of focus in the model eye when the diffractive
lens is off. (b) When the diffractive lens is activated, the object is imaged ens is is
cearly.

Polarized Microscopy. A computer-interfaced polarized optical the optical bench and used to inspect the lenses on a microscopic scale and verify that all electrodes were operating properly. The lens was placed between crossed polarizers $-45^{\circ}$ with respect to the liquid-crystal alignment layer rub direction. For each position on the lens, the intensity seen by the charge-coupled device camera is a function of the voltagedependent phase retardation $\phi(V)$ between the ordinary and extraordinary wave components at the exit surface of the lens. The voltage dependent transmission between crossed polar-
izers $[T(V)]$ is given by

$$
T(V)=\sin ^{2}\left(\frac{\phi(V)}{2}\right) .
$$

[2]

where the transmission is a maximum when $\phi(V)=\pi$ and a

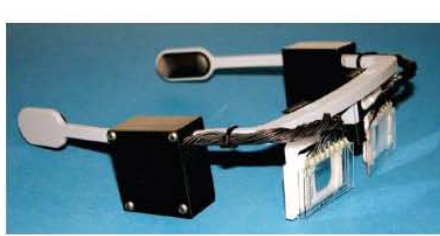

Fig. 4. A prototype of the assembled adaptive eyewear. dependent phase retardation of each electrode can be inspected by observing the intensity warision

Measurement of Diffraction Efficiency. Diffraction efficiency is the amount of light intensity that goes to a particular diffraction
order compared with the sum of intensities in all of the
diffraction orders. To determine the efficiency of the first diffracted order, a linearly polarized $543.5-\mathrm{nm}$ laser beam was xpanded to a diameter of $10 \mathrm{~mm}$ and allowed to pass throug the active area of the lens. The power of the beam was and then again with the lens activated (diffracted power). The ratio of diffracted power to total power is the diffraction

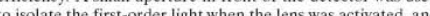
as all measurements were made down-stream of the lens, they were automatically corrected for Fresnel losses, To measure the diffraction efficiency as a function of lens area, a variable aperture (positioned concentrically with respect to the lens)

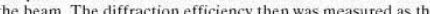
diameter of the beam was increased from 3 to $10 \mathrm{~mm}$ in 1 - $\mathrm{mm}$ increments.

interferometric Testing and MTF Calculation. The performance of (2) nearly polarized $543,5-\mathrm{nm}$ laser source $(24,25)$. The lens under est was placed in the object arm of the interferomerer and the imaged onto a charge-coupled device camera such that the captured interference patterns were formed by the converging plane wave A small aperture was placed between the imaging ens and the camera at the point of focus to isolate and test only he wavefront generated by the first diffracted order. Multiple $\pi / 2$ phase shifts were generated in the reference arm by using piezodoric insducer actuated mirror, and a phase unwrapdiffracted wavefront From this unwraped phase map of the wavefront immediately behind the diffractive lens, the focal length $(f)$ of the diffractive lens is calculated by using

$$
f=\frac{\rho^{2}}{2 O P D}
$$

where OPD is the peak-to-valley optical path difference from center to edge, and $\rho$ is the radius of the test area (26). Because the lenses were designed for operation at $555 \mathrm{~nm}$ but tested at $543.5 \mathrm{~nm}$.

$$
f(\lambda)=f_{0} \frac{\lambda_{0}}{\lambda},
$$

where $f_{0}$ and $\lambda_{0}$ are the design focal length and wavelength, respectively, and $\lambda$ is the measurement wavelength (27). Measurement of the peak-to-valley and rms errors in the wavefro
were made subsequent to removing the best-fit sphere and any tilt from the phase map.

The imaging performance of the lens can be evaluated in modulation to the object modulation at all frequencies. The wavefront of the first-order diffraction can be expressed by a sum of Zernike polynomials (28), and the MTF thus can be calculated by normalized autocorrelation of the generalize pupil function (26)

We thank the Technology and Research Inititative Fund program of the State of Arizona.

PNAS | Aprill 18, 2006 | vol. 103 | no. 16 | 6103 


\section{Lenterne}

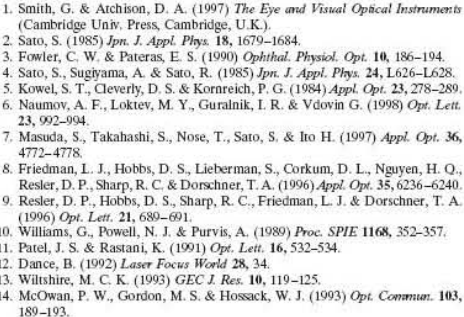

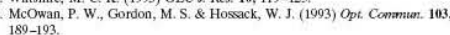

15. Ren, H, Fan, Y.-H. \& Wu, S.T. (2003) Appl. Phys. Lett. 83, 1515-1517. 16. Laude, V. (1998) Opt. Comm 153, 134-152.
17. Hain, M., Glockner, R, Bhattacharya, S., Dias, D., Stankovic, S. \& Tschudi,

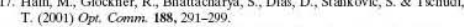
18. Kress, B \& \& Mey, P. (2000) Digital Diffractive Optics (Wiley, New York).
19. Farn, M. W. \& Veldkamp, W. B (1904) in Hardbook of Optics, ed. Bass, M. (McGraw-Hill, New York, Vol. II, Chap. 8, p. 8.15.
20. Brinkley, P. F., Kowel, S. T. \& Chu, C. (1088) Appol Opo. 27, 4578-4586. 21. Apter, B., Efron, U. \& Bahat-Treidel, E. (2004) Appl. Opt. 43, 11-19. 22. Faklis, D. \& Morris, G. M. (1905) Appl Cph J4, 2462-2468. 23. Wu, S. T, Efron, U. \& Hess, I. D. . 1984 A Appp Opt 23, $3911-3015$.
24. Williby, G. (2003) Ph. D. dissertation (Oppical Sciences Center, Univ, of 25. Arizona, Tucson, AZ).

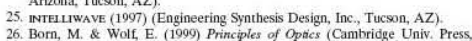
Cambridge, U.K.), 7th Ed.

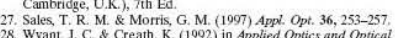
28. Wyant, J.C. \& Creath, K. (1992) in Applied Optics and Optical Engineering.
eds. Shannon, R. R. \& Wyant, J. C. (Academic, London), Vol. XI, Chap. 1 eds. S. 


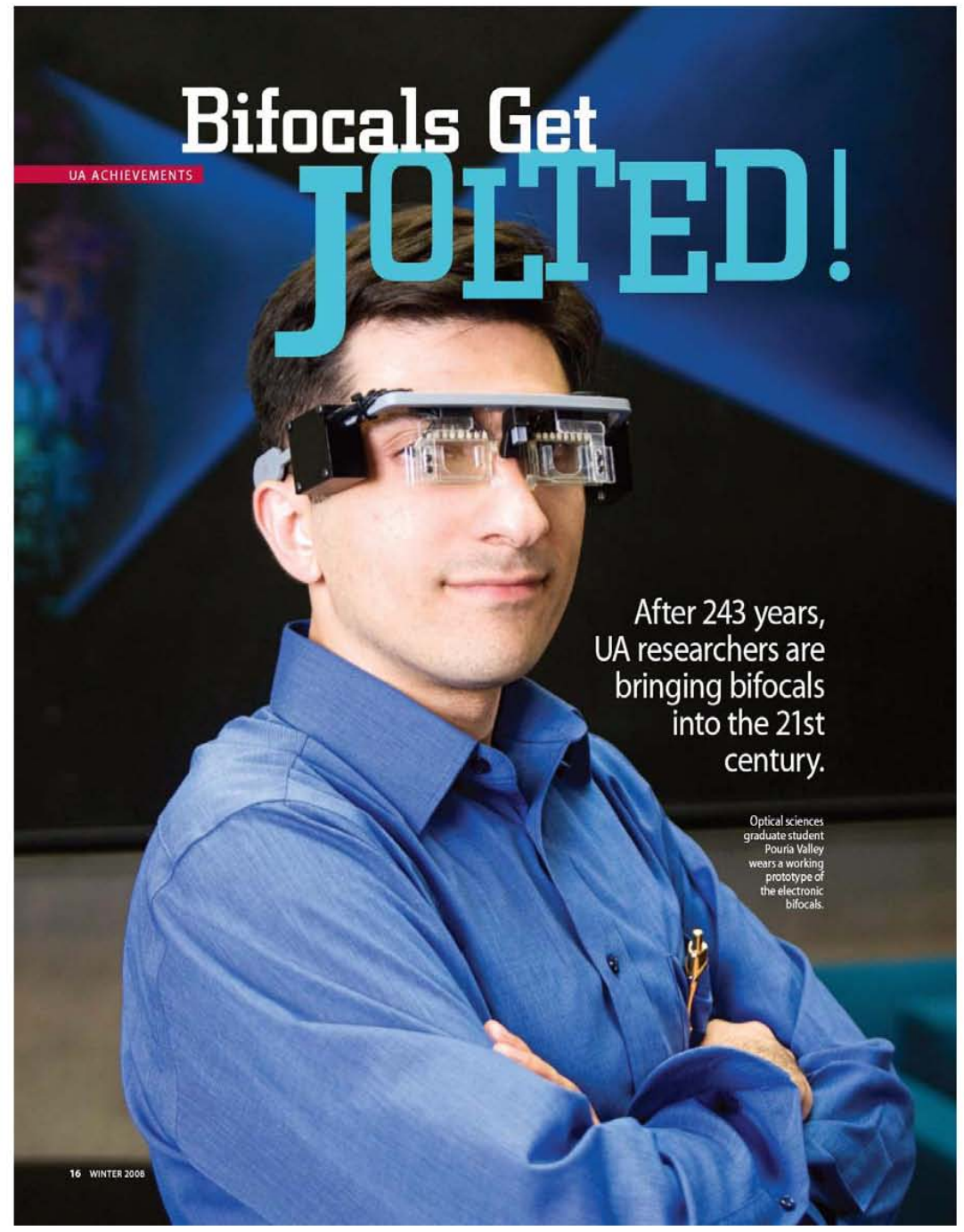


Lenctense

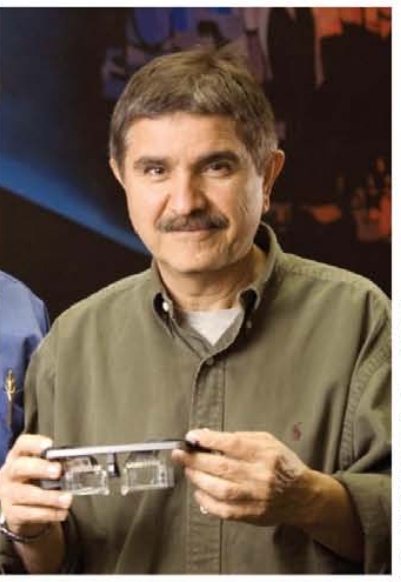

says."There are a loo of fappliz cations for such autofocus lenses, not just eyeglasses.

UA opcical sciences labs, 1 came upon a protorype of barian would have made quite a team.

lin was frus trated by both poor $s$ the eyeglasses of his day. So is Peyghambarian, pronoun pry-gam-BEAR-yan, of

Franklin's invention of bifocals

UA Professorof of Optical Sciences Nasser Peyghambarian

pieces. When you press a

liquid crystal film's structure

alters, adjusting the distance

of focus. The contraption is

in 1764 was one step forward, but

all too well. If

with problems that any bifocal-wearer knows all too well. If

a wall calendar and a cardinal in the tree, something's going to be blurry.

If Franklin were to visit the UA optics labs today, he might be thrilled to put on a pair of fururistic electroactive spees that shift from near strength to far in a flash

With them. Franklin could glance up at a top shelf in the rocery store without ritting his head back to see through the thick lenses of bifocals. He could see flowers by the sidewalk withour droping hin 50 chess. $\mathrm{H}_{\mathrm{e}}$ would be the enwy of without dropping chins thest. He would be the envy of who endure the limits of ordinary bifocals.

Franklin is credited with kicking off the bifocal era by ordering an oprician in London to chop two sers of lenses in ordering an optician in London to chop two sers of
half and make bifocals, said to be Americas first.

Now, the Peyghambarian research team at the UA offers the first full-size lenses embedded with a liquid-crystal film. the first full-size lenses embedded with a liquid-crystal film-
The film sends a small electric voltage to rotate the molThe film sends a small electric voltage to rotate the mol-
ecules and shift the viewing strength from distant to ecules and shift the viewing strength from distant to
near. And his labs are working on electronic continuousnear. And his labs are working on electronic continuous-
focus glasses. "We havent yet demonstrated them, but we focus glasses. "We havent yet demonstrated them, but we
have applied for a patent for our ideas," Peyghambarian

switch and people say, "Wow, I can read." bulky, like goggles. But, Pey-
ghambarian says, you flip the Soon a stylish commercial version will enclose all the miniaturized parts, a tiny computer, batteries, and the rest, inside the earpicece. "It's not clear yet what technology will be used," Peyghambarian says. "The commercial guys will have to figure that our."

The device emerged from six slow years of work by Peyghambarian and ream - including five years of work before they generated a scholarly article (the gold standard of research). But, he had personal motivation to press on "Twe worn bifocals for the hast five years," he says, "and I would rather not. They are a pain.

\section{The effort's premise is simple.}

If light passes through a lens, it curves a bit - the same is true of the eyés lens. A youth can focus as near as two or three inches from the nose. As you age, the lens stiffens. The result is what's called presbyopia, from the Greek word "presbys," or "old person." (Who says age 40 or 45 , when most people face bifocals, is old?

Perhaps as early as the Roman era, people used glass to help correct faulty vision. Now, many centuries later, film of liquid crystal only five microns thick $-\mathrm{a}$ fraction of a human hair, which is about 80 microns - sandwiched

by Ford Burkhart

Photos by Jacob Chinn 
Lemecense

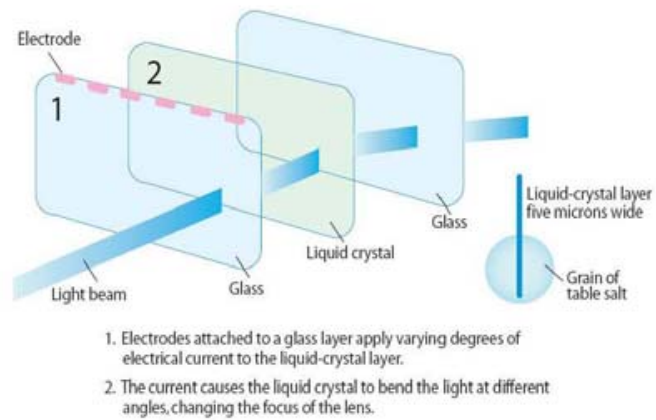

between layers of glass, has been found to offer a more remarkable benefit. This kind of erystal behaves like a liquid, yet the molecules line up like a crystal, making possible inventions like liquid-crystal displays on watches computers, and cell phones. NASA space shuttles use liquid-crystal memory units.

By the 1990s, liquid crystals were a hot solution in search of problems, and the shortcomings of bifocals offered a novel challenge. UA optical scientists knew that a tiny electric voltage - just a few volts, less than what's used for a computer screen - sending currents into multiple transparent electrodes would change the liquid crystal's index of refraction, or power to focus. As a voltage energizes concentric rings of the clear electrodes. the liquid crystal rings act like a lens and refocus the light. Several generations of electro-optic eyeglasses were tested at UA Labs, perfected, and finally handed off to the private sector.

PixelOptics, based in Roanoke, $V_{2}$., is working up a consumer version of what it calls" super vision," due on the market in 2008. Fancy furure versions will be trifocals, Peyghambaria says. The cost may ini-

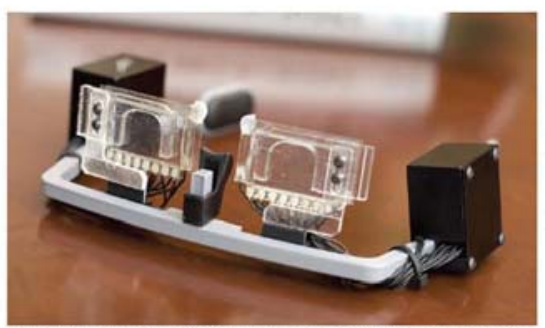

Pixel Optics, based in Poanoke Va, is working up a consumet version of what it calls supervision" due on the market in 2008 tially be around $\$ 500$, "not too large for the top of the market.

Continuous-focus electronic specs are a distant possibility that would work like the autofocus feature on a camera. The device might send out a laser beam from the bridge of the nose, measure how fast it bounces back, and calculate the distance. Another idea would involve a tilt switch: when your head rilts down, the close-up switch goes on. Customers may be offered vari. ous ways to switch strength.

electronic spec

distant possibility that

As a safety measure, the default strength will always be "distance." The scientists figure that if the battery fails while youre driving, it's more autofocus feature important to see the road (in "far" mode) than to autofocus feature important to see the
see the speedometer.

The invention's potential economic impact is staggering. If, as expected, dynamic bifocals or trifocals make the old bifocals and progressive lenses obsolete, they could revolutionize an eyecare market estimated worldwide at $\$ 50$ billion.

When the product reaches the market, Peyghambarian says, TIll be the first one in line." And I'll be right behind him, ready to regain the focusing powers of my youth. That cardinal will be waiting. 